\title{
Numerical Modelling of Heat and Mass Transfer in a Loop Heat Pipe Evaporator
}

By

\author{
Patrick G. Boisvert
}

B. Eng., Carleton University

A thesis submitted to The Faculty of Graduate Studies and Research in partial fulfilment of the degree requirements of

Master of Applied Science

Ottawa-Carleton Institute for

Mechanical and Aerospace Engineering

Department of Mechanical and Aerospace Engineering

Carleton University

Ottawa, Ontario, Canada

May 2007

Copyright $(C)$

2007 - Patrick G. Boisvert 


$\begin{array}{ll}\begin{array}{l}\text { Library and } \\ \text { Archives Canada }\end{array} & \begin{array}{l}\text { Bibliothèque et } \\ \text { Archives Canada }\end{array} \\ \begin{array}{l}\text { Published Heritage } \\ \text { Branch }\end{array} & \begin{array}{l}\text { Direction du } \\ \text { Patrimoine de l'édition }\end{array} \\ \begin{array}{l}\text { 395 Wellington Street } \\ \text { Ottawa ON K1A 0N4 }\end{array} & \begin{array}{l}\text { 395, rue Wellington } \\ \text { Ottana ON K1A ON4 } \\ \text { Canada Oa }\end{array}\end{array}$

Your file Votre référence ISBN: 978-0-494-26982-4 Ourfile Notre référence ISBN: $978-0-494-26982-4$

NOTICE:

The author has granted a nonexclusive license allowing Library and Archives Canada to reproduce, publish, archive, preserve, conserve, communicate to the public by telecommunication or on the Internet, loan, distribute and sell theses worldwide, for commercial or noncommercial purposes, in microform, paper, electronic and/or any other formats.

The author retains copyright ownership and moral rights in this thesis. Neither the thesis nor substantial extracts from it may be printed or otherwise reproduced without the author's permission.
AVIS:

L'auteur a accordé une licence non exclusive permettant à la Bibliothèque et Archives Canada de reproduire, publier, archiver, sauvegarder, conserver, transmettre au public par télécommunication ou par l'Internet, prêter, distribuer et vendre des thèses partout dans le monde, à des fins commerciales ou autres, sur support microforme, papier, électronique et/ou autres formats.

L'auteur conserve la propriété du droit d'auteur et des droits moraux qui protège cette thèse. $\mathrm{Ni}$ la thèse ni des extraits substantiels de celle-ci ne doivent être imprimés ou autrement reproduits sans son autorisation.
In compliance with the Canadian

Privacy Act some supporting forms may have been removed from this thesis.

While these forms may be included in the document page count, their removal does not represent any loss of content from the thesis.
Conformément à la loi canadienne sur la protection de la vie privée, quelques formulaires secondaires ont été enlevés de cette thèse.

Bien que ces formulaires aient inclus dans la pagination, il n'y aura aucun contenu manquant. 
The undersigned recommend to the Faculty of Graduate Studies and Research acceptance of the thesis

\title{
Numerical Modelling of Heat and Mass Transfer in a Loop Heat Pipe Evaporator
}

\author{
Submitted by Patrick G. Boisvert \\ in partial fulfilment of the requirements for the degree of \\ Master of Applied Science
}

Dr. Tarik Kaya, Thesis Supervisor

Dr. Jonathan C. Beddoes, Chair, Department of Mechanical and Aerospace Engineering

Carleton University

May 232007 


\begin{abstract}
Within this work, a two-dimensional steady state loop heat pipe evaporator numerical model was developed. This model solved the diffusive thermal equation in the casing and meniscus, the convective-diffusive thermal equation, the Laplace equation and Darcy flow equation in the wick. Temperature and flux conservation coupled the domains at their respective interfaces. To couple the fluid flow and thermal equations, an iterative process was developed. A finite element solver was utilized for the method of solution. Two internal scenarios using the developed evaporator model were investigated: Scenario 1) - a fully saturated wick and casing; Scenario 2) - the addition of excess fluid in the vapour groove in the form of a meniscus which occurs at low applied heat flux. The results obtained were in good agreement with the available published trends.
\end{abstract}




\section{Dedication}

I dedicate this thesis to my parents, who have instilled in me the belief that anything is possible, the courage to make it happen and the strength to persevere.

\section{To Alain R. Boisvert}

\section{To Louise A. Boisvert}

Merci beaucoup pour tous ce que vous m'avez donné. Je vous aime gros! 


\section{Acknowledgements}

I would like to express my deepest gratitude to my supervisor Dr. Kaya for taking me as one of his students, giving me the opportunity to expand my knowledge and for his guidance and support. Without him, this research would not have been possible. I would also like to thank Dr. Gauthier whom I had the great opportunity to work for on many occasions and is solely responsible for introducing me with his full support to Dr. Kaya thus indirectly is also part of the equation.

During my research at Carleton, I have also made many new acquaintances of which many have become great friends and an indispensable part of the journey. For all the support, the laughs, the good times, the beer-o-clock, the late nights stuck in some computer lab, I thank you from the bottom of my heart. My sanity is owed to you all! A special mention is well deserved by David Cerantola and Erin MacDonald who in their kindness have helped in the revision of this thesis.

Furthermore, I owe a great debt of gratitude to parents Alain and Louise Boisvert for strength and continuous support through this and all of my endeavours, to my little sister Véronique Boisvert for a strong insight on determination, to my little brother Zachary Boisvert who has showed me time and time again there is always room for a laugh, to my grandmother Marthe Gosselin for her kind words of encouragement, to my grandfather Gilles Gosselin for his ceaseless and most times priceless words of wisdom. To the rest 
of my family unmentioned here but not forgotten. And finally, I owe so much to my old friends and loved one who have always been there when I needed them most without hesitation and have understood when I disappeared for days and sometime weeks or months on end without disowning me.

Words cannot express my true feeling. Thank you all! (Merci à tous!) 


\section{Table of Contents}

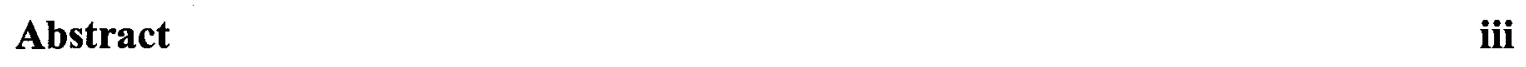

Dedication $\quad$ iv

Acknowledgements $\quad$ v

Table of Contents vii

List of Tables $\quad$ x

List of Figures $\quad$ xi

Nomenclature $\quad$ xiii

Chapter 1: Introduction $\quad 1$

1.1 Background - Heat Pipes.......................................................................... 1

1.1.1 Brief Heat Pipe History and Basic Principles ........................................... 1

1.1.2 Heat Pipe Working Components ............................................................... 3

1.1.3 Operational Limitations of Conventional Heat Pipes .................................. 6

1.2 Background - Loop Heat Pipes ..................................................................... 11

1.2.1 Brief Loop Heat Pipe History and Basic Principles...................................... 11

1.2.2 Heat Pipe Working Components ............................................................... 13

1.2.3 Limitations of the Global Operation of the Loop Heat Pipe.......................... 15

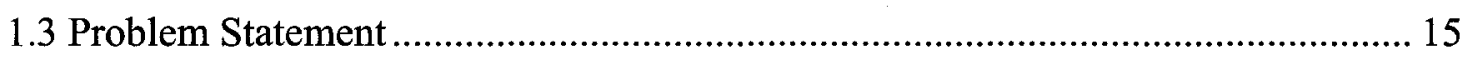

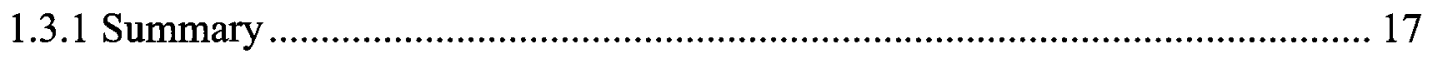

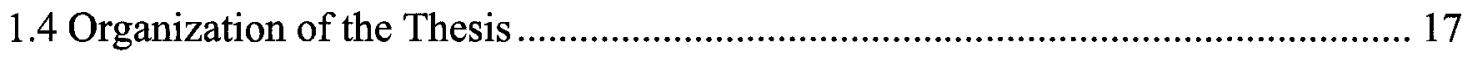

vii 


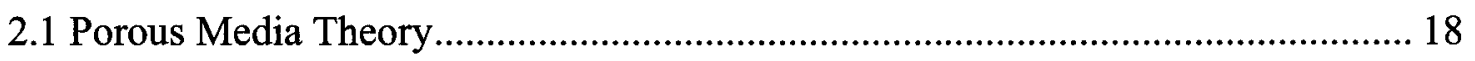

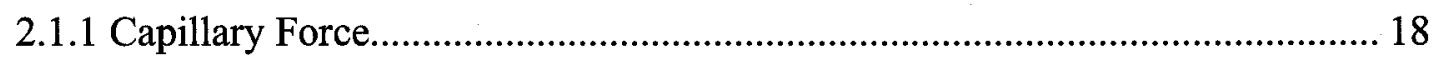

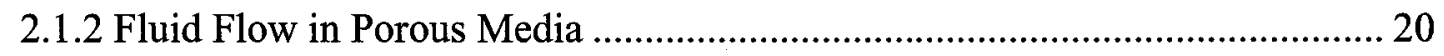

2.1.3 Thermal Transport in Porous Media ............................................................... 22

2.2 Loop Heat Pipe Evaporator Models.................................................................. 26

2.2.1 Evaporator model of Domidov and Yatsenko (1994) ....................................... 28

2.2.2 Evaporator model of Cao and Faghri (1994) .................................................. 29

2.2.3 Evaporator model of Khrustalev and Faghri (1995) ........................................ 30

2.2.4 Evaporator model of Figus et al. (1999) .......................................................... 31

2.2.5 Evaporator model of Kaya and Goldak (2006)................................................ 32

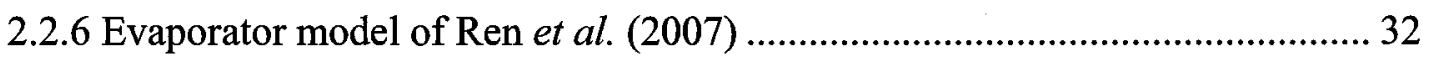

2.2.7 Literary Trends and Conclusions .................................................................. 33

Chapter 3: Computational Model Formulation 35

3.1 Diffusion Thermal Sub-Model.............................................................................. 38

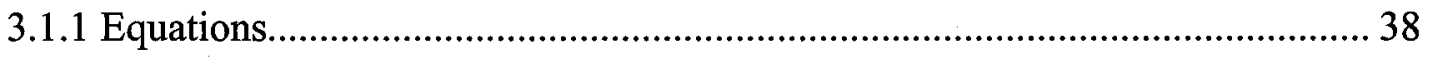

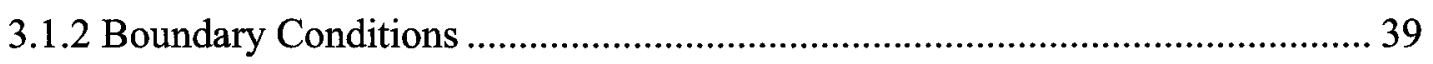

3.2 Convection-Diffusion Thermal Sub-Model......................................................... 41

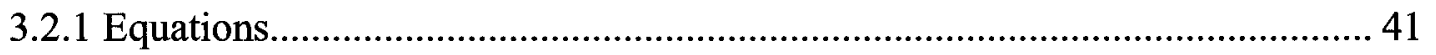

3.2.2 Boundary Conditions ................................................................................ 42

3.3 Laplacian Pressure Sub-Model .......................................................................... 42

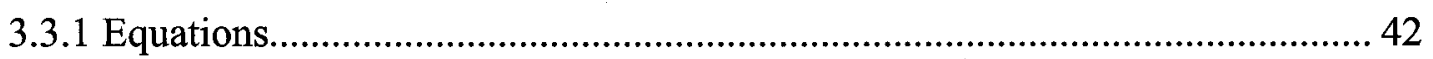

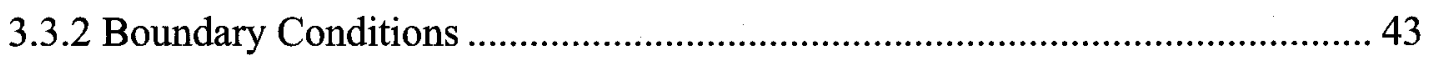

3.4 Darcian Velocity Sub-Model ................................................................................ 44

3.5 Addition of Fluid Meniscus ................................................................................. 45

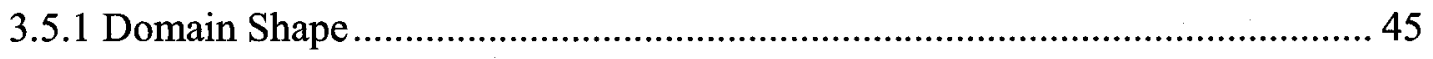

3.5.2 Boundary Condition Alterations .................................................................. 46

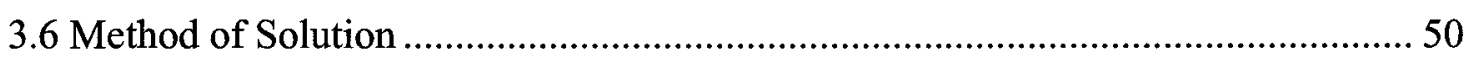

viii 


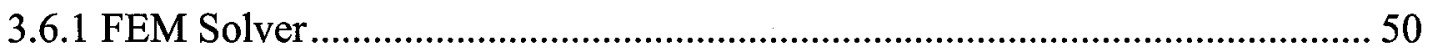

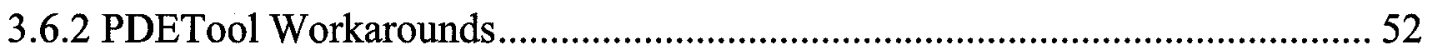

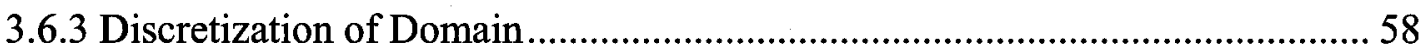

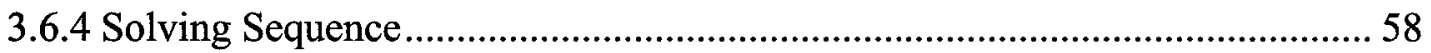

Chapter 4: Benchmark of Model $\quad 63$

4.1 Porous Channel Flow with Partial Heated Surface ...............................................63

4.2 Qualitative Verification of the Evaporative Surface..........................................67

Chapter 5: Loop Heat Pipe Evaporator Model and Results $\quad 71$

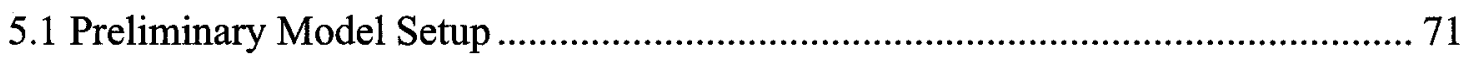

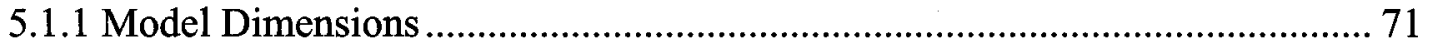

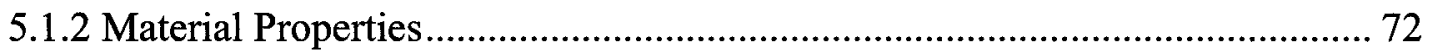

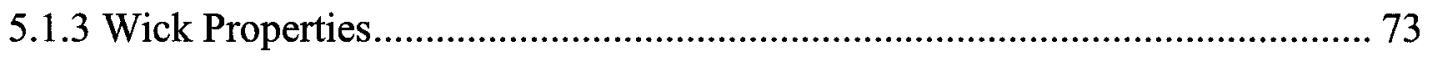

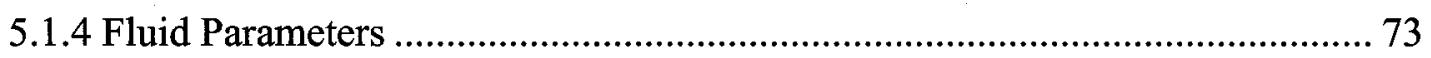

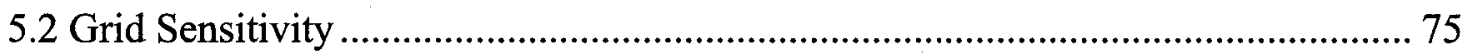

5.3 Scenario 1 (Evaporator with Saturated Wick) .................................................. 78

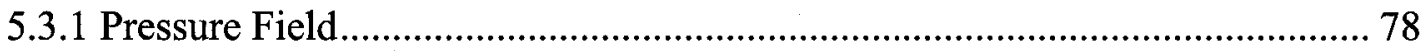

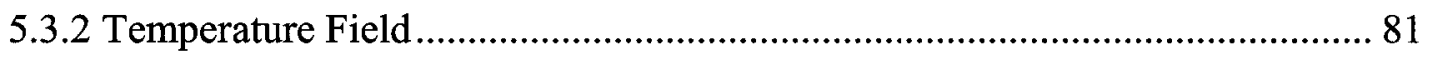

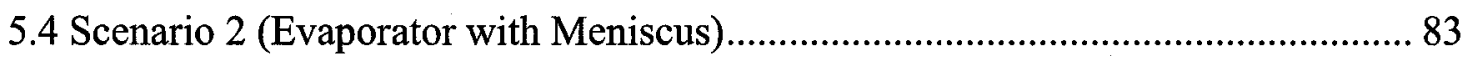

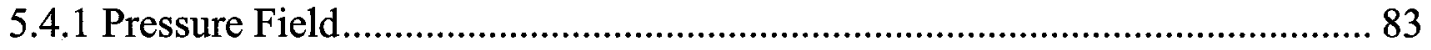

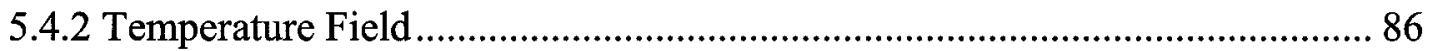

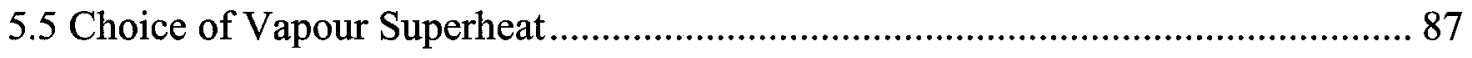

$\begin{array}{ll}\text { Chapter 6: Conclusions } & 89\end{array}$

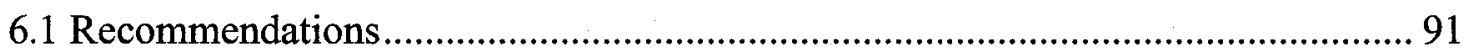

$\begin{array}{ll}\text { References } & 92\end{array}$

ix 


\section{List of Tables}

Table 1-1 Common working fluid and temperature range............................................ 4

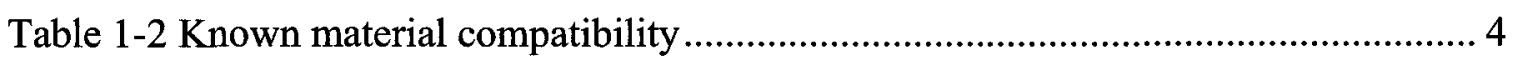

Table 1-3 Parameters of common heat pipe wick structure........................................... 6

Table 1-4 Loop heat pipe wick/evaporator type ....................................................... 14

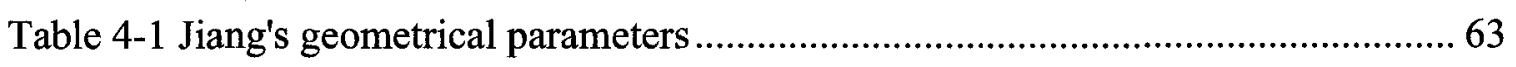

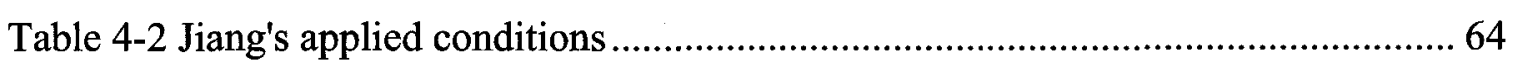

Table 4-3 Effective thermal conductivity for Jiang's problem ....................................65

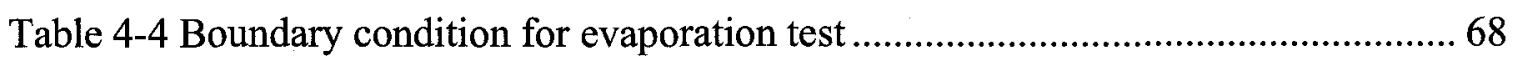

Table 5-1 Dimensions of numerically investigated domain ....................................... 72

Table 5-2 Material properties............................................................................. 73

Table 5-3 Saturated water temperature and pressure ............................................... 74 


\section{List of Figures}

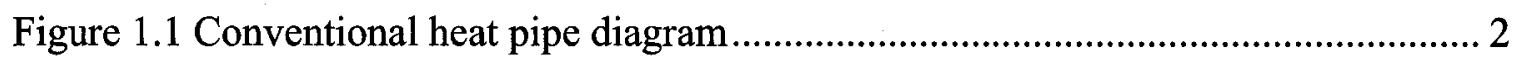

Figure 1.2 Longitudinal vapour and liquid pressure distribution demonstrating necessary

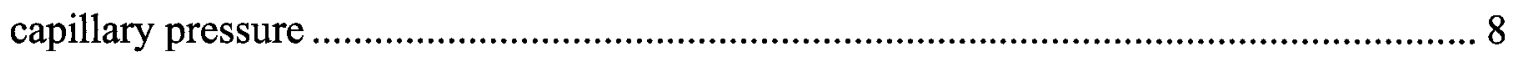

Figure 1.3 Flow comparison to converging-diverging effects......................................... 9

Figure 1.4 Vapour shear force reducing liquid return..................................................... 10

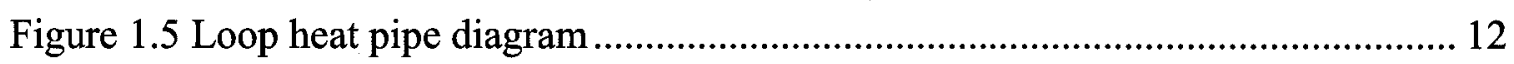

Figure 2.1 Meniscus curvature as a function of evaporation rate .................................... 19

Figure 2.2 Meniscus geometry at liquid-vapour interface............................................. 19

Figure 2.3 Basic phenomena occurring in loop heat pipe operation.................................. 27

Figure 2.4 Evaporator studied by Khrustalev and Faghri ................................................ 31

Figure 3.1 Evaporator cross-sectional cut demonstrating computational domain............. 35

Figure 3.2 Computational domains ........................................................................ 38

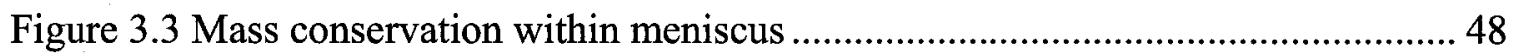

Figure 3.4 Surface normal gradient calculation schematic.............................................. 49

Figure 3.5 Temperature and flux trend on an evaporative surface using a convective

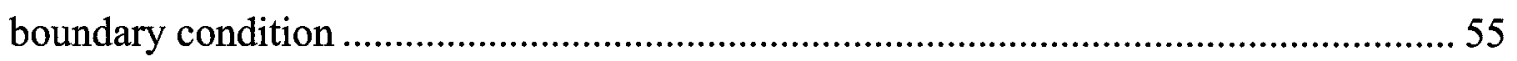

Figure 3.6 Y-component of thermal flux along meniscus surface................................... 57

Figure 3.7 X-component of flux along meniscus-fin interface....................................... 57

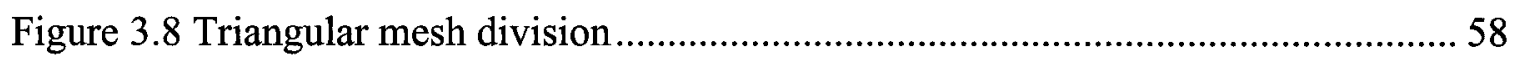

Figure 3.9 Numerical solution sequence flow charts (Part 1 of 2) .................................... 61

Figure 3.10 Numerical solution sequence flow charts (Part 2 of 2) ................................. 62

Figure 4.1 Schematic of lengthened porous channel domain ........................................... 65

Figure 4.2 Temperature field of Jiang's trial 3 .............................................................. 66

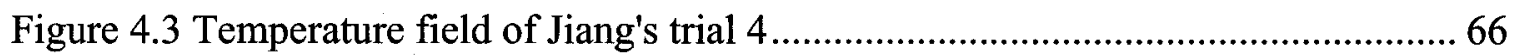

$\mathrm{xi}$ 
Figure 4.4 CFX and evaporator model temperature trend comparison

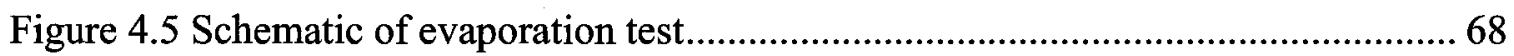

Figure 4.6 Temperature distribution of evaporation test .............................................69

Figure 4.7 Liquid velocity and thermal flux at the evaporative surface of the evaporation

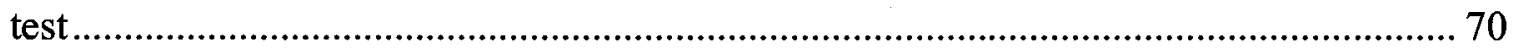

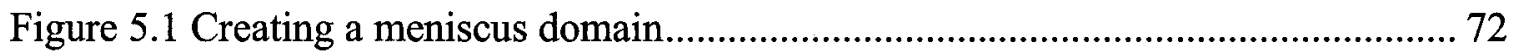

Figure 5.2 Prescribed vapour pressure and saturation temperature ............................. 74

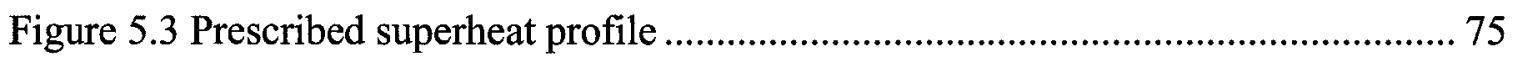

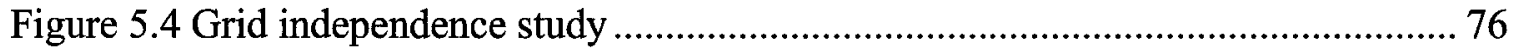

Figure 5.5 Mesh for $30 \mathrm{~W}$ applied heat flux without meniscus..................................... 77

Figure 5.6 Mesh for $30 \mathrm{~W}$ applied heat flux with meniscus.......................................... 77

Figure 5.7 Pressure drop in the wick for the saturated wick......................................... 79

Figure 5.8 Velocity field as determined from pressure drop ...................................... 80

Figure 5.9 Temperature field for the saturated wick case ........................................ 82

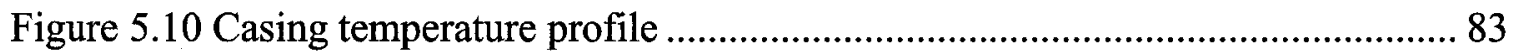

Figure 5.11 Pressure field with presence of meniscus ............................................. 84

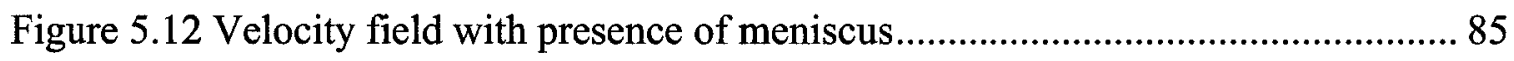

Figure 5.13 Liquid velocity at evaporative surface comparison....................................8 85

Figure 5.14 Temperature field with meniscus ..................................................... 86

Figure 5.15 Investigation of thermal flux in region meniscus ................................... 87

Figure 5.18 Effect of superheat trend on casing temperature ..................................... 88 


\section{Nomenclature}

a MATLAB Partial Differential Equation Tool (PDETool) coefficient

$A \quad$ surface area, $\left[\mathrm{m}^{2}\right]$

$B$ coefficient in Zehner effective conductivity correlation

c PDETool coefficient

C material specific heat capacity, $[\mathrm{kJ} / \mathrm{kg}-\mathrm{K}]$

$C_{\mathrm{p}} \quad$ is the fluids specific heat capacity at constant pressure, $[\mathrm{kJ} / \mathrm{kg}-\mathrm{K}]$

$f \quad$ PDETool coefficient

$g \quad$ PDETool coefficient

$h \quad$ PDETool coefficient

$h_{\mathrm{fg}} \quad$ latent heat of evaporation, $[\mathrm{kJ} / \mathrm{kg}]$

$H_{\mathrm{w}} \quad$ wick height, [m]

$K \quad$ permeability, $\left[\mathrm{m}^{2}\right]$

$\mathbf{K}$ the permeability tensor, $\left[\mathrm{m}^{2}\right]$

$\hat{n} \quad$ the normal unit vector

$P$ pressure, $[\mathrm{Pa}]$

Pe Peclet number, [ ]

xiii 
$P_{\mathrm{H}} \quad$ pressure at point $\mathrm{H}$ (point where fin meets wick), [Pa]

$P_{\infty} \quad$ vapour pressure leaving the wick, $[\mathrm{Pa}]$

$q \quad$ PDETool coefficient

$q \quad$ thermal flux, $\left[\mathrm{W} / \mathrm{m}^{2}\right]$

$q_{\text {in }}$ thermal flux applied to casing, $\left[\mathrm{W} / \mathrm{m}^{2}\right]$

$q_{\mathrm{x}} \quad \mathrm{x}$-component of thermal flux, $\left[\mathrm{W} / \mathrm{m}^{2}\right]$

$q_{\mathrm{y}} \quad \mathrm{y}$-component of thermal flux, $\left[\mathrm{W} / \mathrm{m}^{2}\right]$

$r \quad$ PDETool coefficient

$r \quad$ the radial coordinate

$R \quad$ mean radius of curvature, $[\mathrm{m}]$

$t \quad$ time, $[\mathrm{s}]$

$T \quad$ temperature, $[\mathrm{K}]$

$T^{-} \quad$ solid medium interfacial temperature, $[\mathrm{K}]$

$T^{+} \quad$ fluid medium interfacial temperature, $[\mathrm{K}]$

$T_{\text {sat }} \quad$ saturation temperature, $[\mathrm{K}]$

$T_{\text {vap }} \quad$ vapour temperature, $[\mathrm{K}]$

$u \quad$ PDETool solution parameter

$u_{\mathrm{D}} \quad$ Darcian velocity or filter velocity, $[\mathrm{m} / \mathrm{s}]$

$u_{\mathrm{Dx}} \quad \mathrm{x}$-component of velocity, $[\mathrm{m} / \mathrm{s}]$

$u_{\mathrm{DY}} \quad \mathrm{y}$-component of velocity, $[\mathrm{m} / \mathrm{s}]$

xiv 
$x \quad$ the abscissa

$y \quad$ the ordinate

Greek

$\alpha \quad$ thermal convective coefficient, $\left[\mathrm{W} / \mathrm{m}^{2}-\mathrm{K}\right]$

$\alpha_{\text {evap }}$ thermal convection coefficient of evaporation, $\left[\mathrm{W} / \mathrm{m}^{2}-\mathrm{K}\right]$

$\alpha_{T} \quad$ dimensionless thermal slip coefficient

$\partial x \quad \mathrm{x}$-component of gradient

$\partial x_{\mathrm{n}} \quad$ projection of the $\mathrm{x}$-component of gradient

$\partial y \quad \mathrm{y}$-component of gradient

$\partial y_{\mathrm{n}} \quad$ projection of the y-component of gradient

$\varepsilon \quad$ porosity, []

$\rho \quad$ materials density, $\left[\mathrm{kg} / \mathrm{m}^{3}\right]$

$\lambda$ thermal conductivity coefficient, $[\mathrm{W} / \mathrm{m}-\mathrm{K}]$

$\hbar$ the pore length scale, $[\mathrm{m}]$

$\theta \quad$ wetting angle, [rad]

$\theta \quad$ angular coordinate or polar angle

$\theta \quad$ angle to the vertical axis, [rad]

$\sigma \quad$ surface tension, $[\mathrm{N} / \mathrm{m}]$

$\sigma \quad$ ratio of fluid thermal conductivity to solid

$\mu \quad$ viscosity, $[\mathrm{kg} / \mathrm{m}-\mathrm{s}]$

$\mu^{\prime} \quad$ Einstein effective viscosity, $[\mathrm{kg} / \mathrm{m}-\mathrm{s}]$ 


\section{Subscripts}

c casing parameter

c capillary parameter

e effect porous media parameter

f fluid parameter

m meniscus parameter

s solid parameter

v vapour parameter

w wick parameter

Operations

$\nabla T \quad$ gradient of $T,\left(\frac{\partial}{\partial x} \hat{i}+\frac{\partial}{\partial y} \hat{j}\right) T=\frac{\partial T}{\partial x} \hat{i}+\frac{\partial T}{\partial y} \hat{j}$

$\nabla \cdot u_{\mathrm{D}}$ divergence of $u_{\mathrm{D}},\left(\frac{\partial}{\partial x} \hat{i}+\frac{\partial}{\partial y} \hat{j}\right) \cdot\left(u_{\mathrm{DX}} \hat{i}+u_{\mathrm{DY}} \hat{j}\right)=\frac{\partial u_{\mathrm{DX}}}{\partial x}+\frac{\partial u_{\mathrm{DY}}}{\partial y}$

$\Delta P \quad$ Laplacian of $P,\left(\frac{\partial^{2}}{\partial x^{2}}+\frac{\partial^{2}}{\partial y^{2}}\right) P=\frac{\partial^{2} P}{\partial x^{2}}+\frac{\partial^{2} P}{\partial y^{2}}$

\langle\rangle local averaged value 


\section{Chapter 1: Introduction}

\subsection{Background - Heat Pipes}

\subsubsection{Brief Heat Pipe History and Basic Principles}

In 1836, Jacob Perkins developed a thermal transport device known as the Perkins tube. This device consisted of an evacuated closed tube containing a minute amount of fluid. When a heat load was applied to one end of the tube, the fluid present in this localized region evaporated and traveled to the opposite end of the tube with additional energy where it then condensed releasing its latent heat. If oriented vertically with the condenser above the evaporator, the condensate returned to the heated end of the Perkins tube in a continuous fashion. However, much inefficiency caused by improper orientation makes the use of this primitive device challenging. [1][2]

Roughly a century later, in 1944, Gaugler introduced the basic heat pipe which is still in use today. With the addition of a capillary structure lining the interior of the casing, the working fluid now has a return mechanism needed to keep the heated section fully saturated in most orientations. Many came after Gaugler introducing new capillary structures, new working fluids, and finding new applications. 
The simplicity of the heat pipe can be seen first and foremost in its construction. As seen in Figure 1.1, a heat pipe consists of an evacuated tubular casing usually made of metals or ceramics and charged with a small amount of working fluid. The casing is lined with a capillary wick structure which is the liquid flow path. The hollow center serves as the vapour cavity allowing transport of vapour away from the heat source.

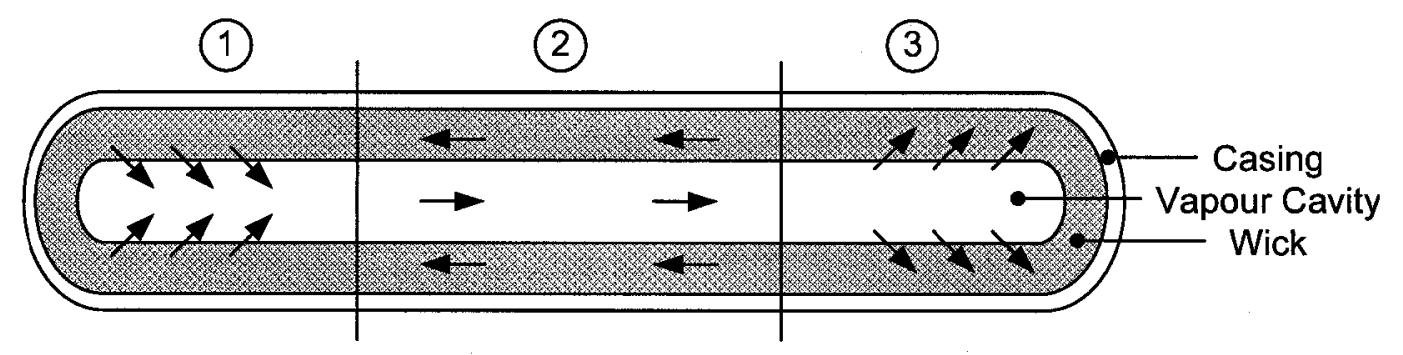

Figure 1.1 Conventional heat pipe diagram

The basic mechanism behind the conventional heat pipe is also very simple. Also seen in Figure 1.1, there are three sections in a heat pipe: 1) the evaporator, 2) the adiabatic section, and 3) the condenser. Heat flux is applied to the evaporator where it traverses the casing into the liquid saturated wick where evaporation occurs. Due to the resulting pressure difference in the core, the introduced vapour will undergo axial acceleration, forcing it through the adiabatic section towards the condenser. Upon entering the condenser section, the vapour decelerates and begins condensing into the wick such that latent heat of evaporation is removed. The condensate then travels counter direction up the wick through capillary force, keeping the evaporator section saturated and thus completing the cycle. [1][2]

Today, the heat pipe is used in a wide range of applications such as electronic cooling, heat exchangers, oven and furnace preheaters, gas turbine cooling, nuclear 
cooling, tool production, de-icing, permafrost stabilization and many more [1][2]. Heat pipes are attractive for use in these applications because they can typically transport several orders of magnitude more heat than options such as solid metal conductors or conventional convective systems (finned plates and fans)[1]. Another aspect of the heat pipe which is appealing is that the capillary force needed for the fluid return is selfregulating through the natural change of the fluid meniscus' curvature within the wick to different heat loads [3]; this aspect will be discussed in detail in Section 2.1.1. Finally, a heat pipe has no moving parts and requires no external power to work; therefore, maintenance is not required which is beneficial when replacement is impossible such as in space applications.

\subsubsection{Heat Pipe Working Components}

The working fluid and wick are of utmost importance for the heat pipe's effectiveness. Choosing the right combination for the application can be somewhat of a meticulous art.

\section{Working Fluid}

To maximize the heat pipe performance, the proper choice of working fluid is critical. Every available choice of working fluid has a specific working temperature range that is usually dictated by the temperature at which the saturation pressure is situated between $0.1 \mathrm{~atm}$ and $20 \mathrm{~atm}$ [2]. Table 1-1 summarizes common working fluids along with their useful temperature range. The working fluid must also be compatible with the casing and wick materials to prevent chemical reaction or galvanic degradation [2]. Fluids and materials with known compatibility are summarized in Table 1-2. 
Table 1-1 Common working fluid and temperature range (Adapted from [2])

\begin{tabular}{lcc} 
Working Fluid & $\begin{array}{c}\text { Boiling Point } \\
\text { @ } \mathbf{~ a t m , ~ [ K ] ~}\end{array}$ & $\begin{array}{c}\text { Useful Range, } \\
{[\mathrm{K}]}\end{array}$ \\
\hline Helium & 4.21 & $2-4$ \\
Hydrogen & 13.8 & $14-31$ \\
Freon 22 & 232.2 & $193-297$ \\
Ammonia & 239.9 & $213-373$ \\
Acetone & 329.4 & $273-393$ \\
Water & 373.1 & $303-473$ \\
Potassium & 1032 & $773-1273$ \\
Sodium & 1151 & $873-1473$ \\
Lithium & 1615 & $1273-2073$ \\
\hline
\end{tabular}

Table 1-2 Known material compatibility (Adapted from [2])

\begin{tabular}{lll}
\hline Working Fluid & \multicolumn{1}{c}{ Compatible Material } & \multicolumn{1}{c}{ Incompatible Material } \\
\hline \hline Ammonia & Aluminium, SS, Cold & Copper \\
& rolled steel, Iron, Nickel & \\
Acetone & $\begin{array}{l}\text { Aluminium, SS, Copper, } \\
\text { Brass, Silica }\end{array}$ & \\
Water & SS, Copper, Silica, Nickel, & Aluminium, Inconel \\
& Titanium & \\
Sodium & SS, Nickel, Inconel, & Titanium \\
& Niobium & \\
Lithium & Tungsten, Tantalum, & SS, Nickel, Inconel, \\
& Molybdenum, Niobium & Titanium \\
\hline
\end{tabular}

Another very important criterion is that the working fluid possesses a high surface tension. A greater surface tension will lead to greater capillary pumping capabilities of the wick [2]. Finally, a large latent heat of vaporization is needed to increase the heat transportation efficiency. 
The amount of working fluid used to charge the heat pipe is also of great concern. For proper operation, the wick in the evaporator section must remain saturated at all times. As evaporation occurs, the fluid recedes into the wick, which may reduce the maximum heat transfer if the fluid recedes excessively [1]. If compensated with an overabundance of working fluid, the excess will gather at the condenser section increasing its thermal resistance thus lowering the condenser efficiency [2]. Subsequently, choosing the proper amount of fluid to maintain equilibrium is difficult to determine.

\section{Wick Structures}

The purpose of the wick is to provide a passage for the return of the fluid from the condenser to the evaporator through capillary force. Therefore, the porous media parameters will dictate the effectiveness of the wick. At the surface of the porous media (at the liquid-vapour interface or evaporative surface), smaller pores facilitates an increase in available capillary force [2]. However, if smaller pores are found throughout the wick's core, an increase in the flow restriction will be felt by the working fluid [2]. Therefore, since the wick is located throughout the whole heat pipe (for matter of convenience and easier construction), most common wick structures are constructed with single (homogeneous) pore size thus only effectively taking into consideration the increase capillary pressure or decrease in the wicks internal flow restrictions. Since the study of capillary pressure and thermal transport in the wick is essential to the inner workings of the heat pipe, a more detailed summary of porous media transport theory is provided in Section 2.1 . 
Table 1-3 describes three common wick structures with pertinent parameters (available capillary pressure, thermal conductivity and permeability) utilized for heat pipes. Choice of wick is mainly based upon the intended application of the heat pipe. Other wick types exist, but are merely adaptations or combinations of the basic three.

Table 1-3 Parameters of common heat pipe wick structure (Adapted from [2])

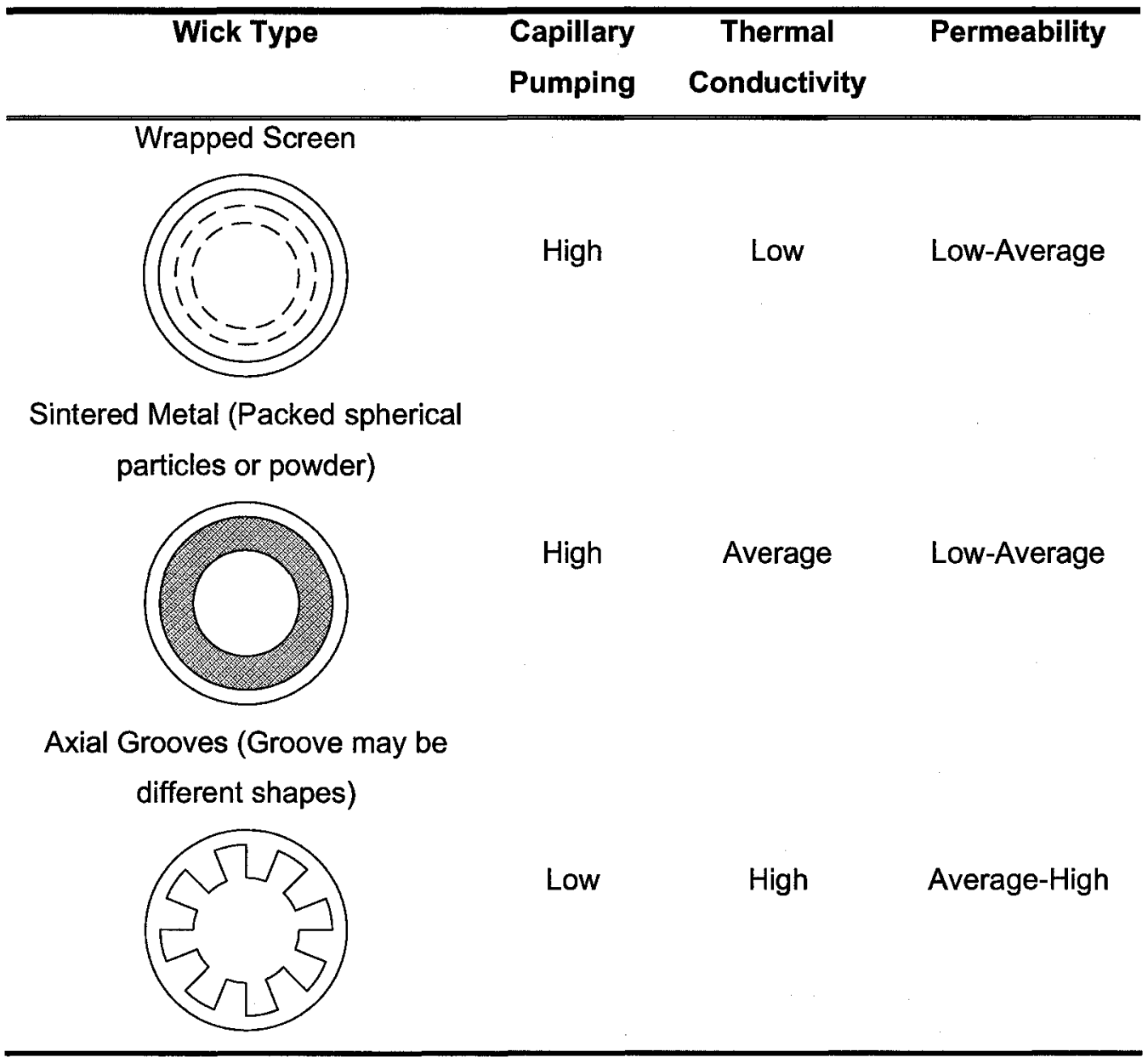

\subsubsection{Operational Limitations of Conventional Heat Pipes}

Even though the basic mechanism within the conventional configuration is straightforward there are many complicated phenomena that can limit or inhibit the heat 
pipe's working capability, all of which originate from the laws of general fluid mechanics and heat transfer within porous media [2]. As such, the circulation capability of the working fluid is the most important factor when examining the heat transfer limitations. This circulation is limited by four distinct mechanisms: the maximum capillary performance of the wick (capillary limitation), choking of vapour within the vapour core (sonic limitation), shear forces occurring at the interface due to relative velocities of the vapour and liquid phases (entrainment limitation), as well as blockage of liquid flow in the wick due to the onset of boiling (boiling limitation) [1][2].

\section{Capillary Limitation or Hydrodynamic Limit}

The working fluid undergoes a pressure drop in both the vapour core and porous media in the opposite direction along the heat pipe because of the liquid and vapour counter flows. Being that the pressure gradients of the liquid and vapour are not of the same magnitude along the heat pipe (see Figure 1.2), the available capillary pressure created by the wick and working fluid combination must overcome the largest magnitude difference. If the capillary pressure cannot compensate for the evaporation rate, the heat pipe will experience a wick dry out at the evaporator section which is detrimental to its operation. Aspects that will contribute to the capillary limit are frictional drag within the porous media and gravitational forces. Gravitational forces exert the largest constraint on a conventional heat pipe depending on its positioning and environment. 


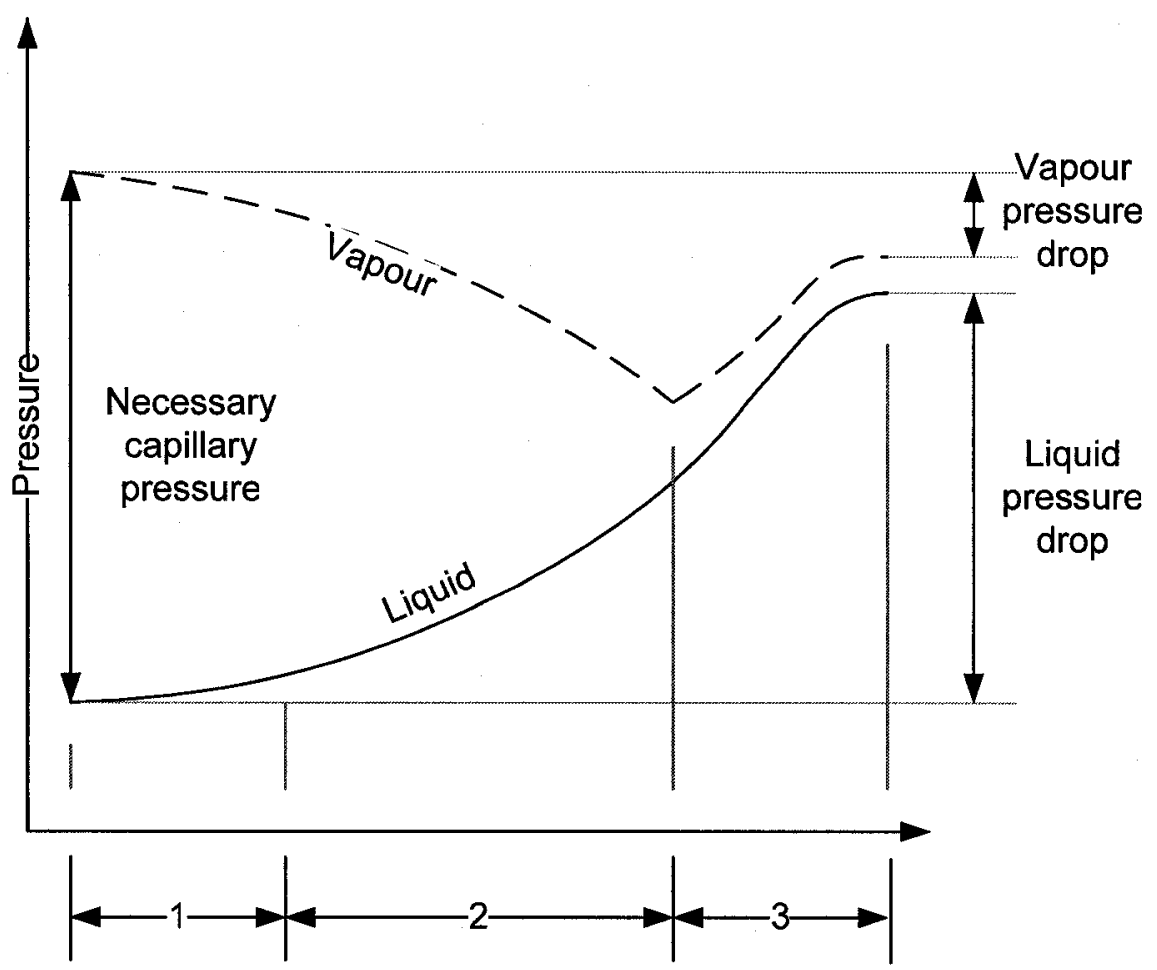

Figure 1.2 Longitudinal vapour and liquid pressure distribution demonstrating necessary capillary pressure: 1 - Evaporator, 2 - Adiabatic section, 3 - Condenser (Adapted from [2])

\section{Sonic Limitation}

The vapour undergoes an addition and removal of mass in the vapour core at the evaporator and condensation sections respectfully, causing accelerations and decelerations in the velocity. Since the addition and removal of vapour occurs in a constant cross sectional area, the vapour will experience the same effect of a convergingdiverging nozzle where the mass flow rate is steady and the area changes [1] as illustrated in Figure 1.3. If the velocity reaches the speed of sound, the massflow will become choked and therefore the heat removal process will be limited (the amount of heat transferred is proportional to the mass flow). Once the flow is choked, the only way to 
increase the mass flow is to increase the temperature of the evaporator. Further increasing the heat rejection only lowers the condenser temperature [1]. However, the sonic limitation is usually only an issue during the start up transient regime or for a liquid-metal heat pipe where the vapour density is very low and velocities are high.

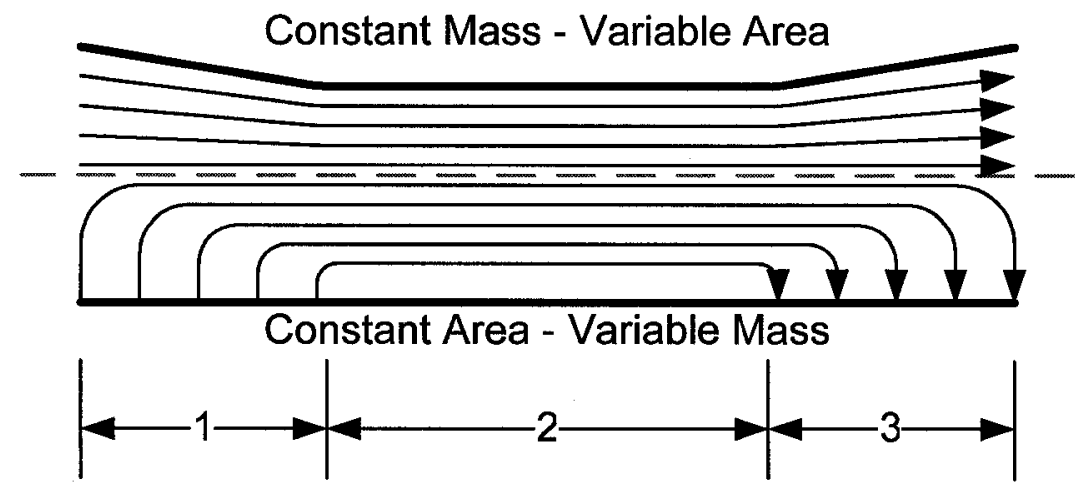

Figure 1.3 Flow comparison to converging-diverging effects: 1 - Evaporator, 2 - Adiabatic section, 3 - Condenser

\section{Entrainment Limitation}

Due to the liquid and vapour motion in opposite direction, the liquid experiences a surface sheer effect at the liquid-vapour interface [1]. At high vapour velocities, the shear force can become large enough to counteract the capillary force of the wick thereby eliminating the fluid flow as illustrated in Figure 1.4. Without a fluid return to the evaporator, the wick will experience a dry out. Even though the vapour velocity dictates whether this limitation will occur, wicks with small pores at the liquid-vapour interface will generally increase the entrainment limitation [2]. 


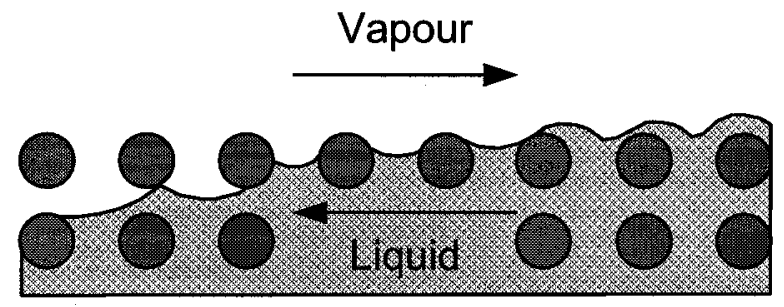

Figure 1.4 Vapour shear force reducing liquid return

\section{Boiling Limitation}

The primary mechanism of a heat pipe is evaporation of the working fluid from the wicking structure due to a flux applied at the evaporator section. There is however a disadvantage inherently built into the heat pipe with the conventional configuration. The heat is applied through the porous media to evaporate on the opposite side. If the certain level of flux is exceeded, then boiling may start to occur within the porous media thus lowering effectiveness of the capillary force and lowering the effective heat transfer coefficient of the wick and working fluid combination creating hot spots [1]. The boiling limitation is the limit where the maximum heat flux absorbed creates no vapour bubbles in the evaporator section, which is usually associated with non-metallic working fluid and will be decreased by using wicks of large effective conductance [2].

\section{Other Limiting Factors}

There are also many other limiting factors which include vapour pressure limit, vapour continuum limit, condenser heat transfer limits, and frozen startup limit [2]; however, these limitations only come into consideration when looking at the transient state of operation of a heat pipe which is outside the scope of this study. 


\subsection{Background - Loop Heat Pipes}

\subsubsection{Brief Loop Heat Pipe History and Basic Principles}

In many cases the conventional heat pipe described in Section 1.1.1, is sufficient or excels for thermal transport tasks. However, the conventional heat pipe has many limitations as discussed in Section 1.1.3 of which its orientation in a gravitational field is of greatest concern and sometimes cannot be avoided. Therefore, in the early $1980 \mathrm{~s}$, an alternative was developed at the Laboratory of Heat Transfer Devices and patented by Maidanik in 1985 [4]. This device is known as the loop heat pipe (see Figure 1.5 for a simplified diagram). Most of the considerable research and developments of the loop heat pipe only started in the 1990s when a few reliable prototypes were produced. All available results from these prototypes indicated that the benefits of the conventional heat pipe were retained with the added gain that most of the limitations are reduced and most importantly, it is capable of operating in any orientation. The only great disadvantage of the loop heat pipe is that there still exists a lack of available experimental data on the internal operation of each component due to the great difficulty of instrumenting the evacuated device without disturbing the flow [2].

The basic design of the loop heat pipe is slightly more complicated than that of the conventional heat pipe. As shown in Figure 1.5, the loop heat pipe consists of the same three sections as in the conventional heat pipe, the evaporator, the adiabatic section and the condenser (sections 1, 2 and, 3 respectively). In this case however, the evaporator consists of an encased localised high performance wick constructed in a fashion to restrict flow motion to one direction (diodized flow). Also, there may be several 
evaporators just as there may be several condensers (not illustrated in the diagram) in the loop heat pipe. This addition of evaporators and/or condensers may be beneficial as they may be used to decrease the overall pressure drop across the overall loop and/or enable multiple heat transport tasks to be conducted simultaneously.

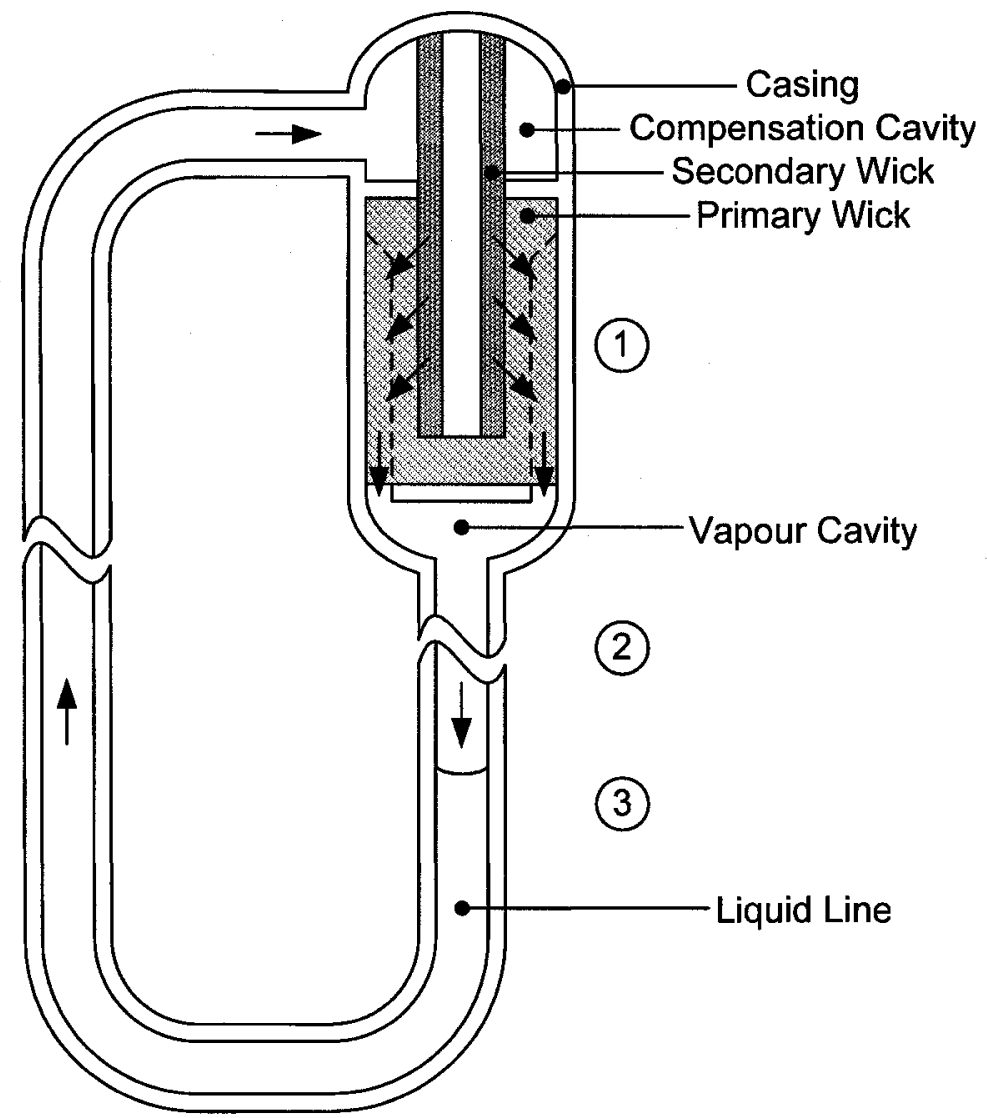

Figure 1.5 Loop heat pipe diagram

Loop heat pipe operation is much more intricate compared to the conventional heat pipe. When a heat load is applied to the evaporator, the flux will travel through the casing to the localised primary wick where evaporation of the local working fluid will occur. However, in the loop heat pipe, the vapour accumulated in the vapour cavity can 
only travel into the vapour line regardless of orientation. As the vapour pressure increases, it will propel the working fluid forward through the return line to the condenser. Once the vapour reaches the condenser, the loop heat pipe is said to be primed and ready for operation as the vapour is now being condensed. The continuous evaporation at the evaporator will cause a constant return of the condensate to the compensation cavity where it will be re-fed to the evaporator through capillary pumping of the secondary wick to complete the cycle.

\subsubsection{Heat Pipe Working Components}

\section{Working Fluids}

The working fluid in the loop heat pipe has the same overall goals and restrictions as that of the conventional heat pipe. Section 1.1.2 summarizes major criteria critical to the proper choice of working fluid to ensure device effectiveness. However, the amount of working fluid introduced in the loop heat pipe is different compared to the conventional heat pipe. A compensation cavity is an integral part of the loop heat pipe and as such more fluid is inherently used. This compensation cavity will self-regulate the amount fluid circulating throughout the device. The surplus fluid becomes essential when large evaporation rates occur [2].

\section{Wick Structures}

The wick structure in the loop heat pipe is localized in the evaporator. This localization allows for the development of high performance wicks and different designs of which two types are displayed in Table 1-4. In both designs, the wick is force-fitted into an 
axially grooved casing. The arrows demonstrate the fluid path. The liquid flows through the core into the porous media which creates the necessary capillary force to propel the liquid towards the casing fin where heat is applied. Subsequently, when evaporation occurs, the vapour will flow into the axial vapour grooves. The capillary force will naturally adjust itself as to match the evaporation and condensation conditions. Again, smaller surface wick pores will produce a higher available capillary force and also increase the heat transport capability.

Table 1-4 Loop heat pipe wick/evaporator type

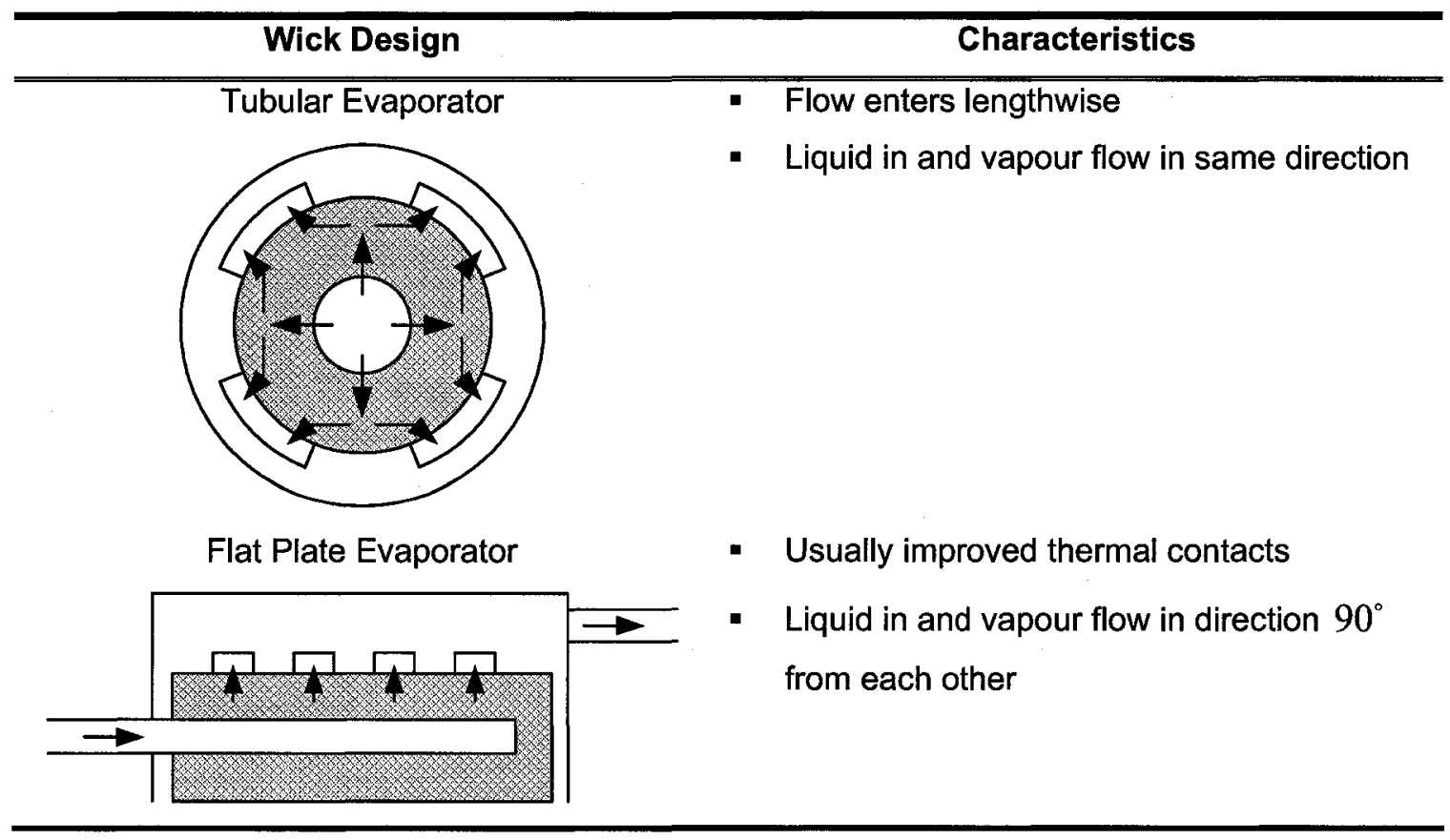

A great benefit of these wick/evaporator designs is that the overall heat transfer is two to three times higher than that of the conventional heat pipe which is a result of the heat addition occurring on the same side as the evaporation [2]. Section 2.1 provides a more detailed summary of fluid flow and heat transfer in porous media. 


\subsubsection{Limitations of the Global Operation of the Loop Heat Pipe}

\section{Capillary Limitation}

Similarly to the conventional heat pipe, a primary concern for the loop heat pipe is the capillary limitation. When capillary pressure is insufficient to provide a constant fluid flow to the casing fin where the thermal flux is applied, the wick will dry out. However, in most cases the fluid is merely only recessed into the porous media, evaporation can still occur though the thermal resistance will increase [2].

\section{Wick Limitation}

A minimum pressure drop is required across the wick so that the fluid flow may be sufficient to displace the liquid from the vapour line into the liquid line to ensure that the loop heat pipe remains primed [2].

\subsection{Problem Statement}

Though research on the conventional heat pipe continues, much of its operating principles are fairly well understood and the available numerical models have undergone several decades of refinement starting with the well publicized 1970s model developed by Chi [1]. As a newer alternative, the loop heat pipe has only seen a couple decades worth of research whereas partial mathematical modelling of the complete loop heat pipe only truly started with the 1D steady state loop heat pipe model published in 1999 by Kaya and Hoang [3]. As previously indicated, the only great disadvantage of the loop heat pipe is the lack of available extensive internal results. Some of its operating principles are still only assumed and have yet to be experimentally verified due to the 
great difficulty of internal measurement. Furthermore, complication arises in the use of some reported experimental data for model validation owing to the simplifications made in the description of parameters and experimental procedures. Therefore, it would be of great benefit to develop experimental setups and numerical models to investigate the internal workings of the loop heat pipe of which the numerical modelling of the evaporator was undertaken as part of this work.

The evaporator model was coded using MATLAB as the base language with intentions of evaluating the internal flow properties. Two different internal scenarios for operation at low heat fluxes will be investigated:

1. Model including a fully saturated wick and casing;

2. Model including a fully saturated wick and casing with the addition of working fluid accumulated within the vapour groove in the form of a meniscus.

These two models include several different sub-models. In the case of thermal analysis, the casing and fluid accumulated are modelled with a pure diffusion thermal model whereas the wick is modelled with a convection-diffusion thermal model. Fluid flow analysis is conducted with a Laplacian pressure model with a Darcian conversion for velocity extraction. The thermal and fluid flow analyses were coupled using an iterative process. The shape of the accumulated fluid within the vapour channel was determined by the model using an iterative process coupling the pressure field with the YoungLaplace equation. 


\subsubsection{Summary}

The objectives of this thesis are:

1. Develop a steady state $2 \mathrm{D}$ evaporator model for low heat fluxes;

2. Simulate results for a range of low heat flux without and with access fluid in vapour cavity;

3. Investigate effects of fluid accumulated within vapour channel.

\subsection{Organization of the Thesis}

This thesis presents the development of two loop heat evaporator models for low heat fluxes are well as results as predicted by these models and is organized as follows:

Chapter 1: Introduction - Introduces the heat pipe and loop heat pipe technology providing both a brief history and background principles. This section also presents the goals of the thesis

Chapter 2: Literature Survey - Presents necessary theory for understanding of evaporator operation as well as a summary of published evaporator models.

Chapter 3: Computational Model Formulation - Presents all governing equations and boundary conditions as well as the developed process for solving these sets of equations.

Chapter 4: Benchmark of Model - Presents results from verification process of the evaporator model.

Chapter 5: Loop Heat Pipe Evaporator Model and Results - Presents all applied parameters used in the model and discusses the derived results.

Chapter 6: Conclusion - Presents the conclusions of the thesis and discusses recommendation for future work. 


\section{Chapter 2: Literature Survey}

\subsection{Porous Media Theory}

To understand the basis of operation of the loop heat pipe's evaporator is to acknowledge that the entire process is driven by evaporation and that this is feasible only through the use of the wick composed of porous medium.

As described by Kaviany [5], the porous media is simply a heterogeneous system made of a solid matrix, solid and void coexisting in one domain, which can be treated as a continuum only if the function of the solid and the fluid permeating the void are well accounted for; this includes effects of capillary force, fluid flow and thermal transport.

\subsubsection{Capillary Force}

As the permeating fluid evaporates from the saturated porous media, the permeating fluid will recede into the solid matrix therefore changing its surface curvature (Figure 2.1 demonstrates the fluid surface curvature as a function of evaporation rate). In general, this increase in the liquid-vapour interfacial area will induce work against the surface tension (which can be thought of as the elastic force) of the permeating fluid. 


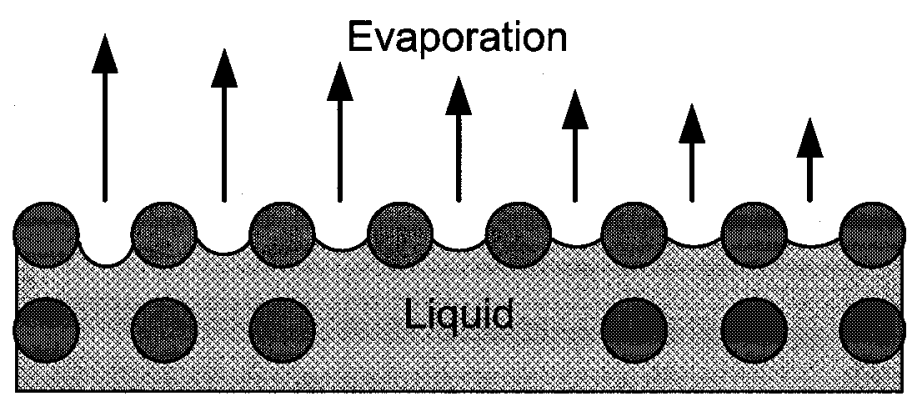

Figure 2.1 Meniscus curvature as a function of evaporation rate

However, the surface tension consisting of surface and near surface ionic force, metallic bonds, hydrogen bonds and the van der Waals forces will naturally desire to return the liquid-vapour interfacial surface to the point of least stored energy (its original shape) thereby increasing the angle (known as the wetting angle) at which the permeating fluid makes with the solid matrix [5] (Figure 2.2 demonstrates the variables dictating the meniscus geometry).

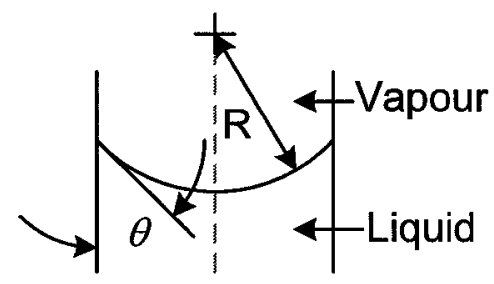

Figure 2.2 Meniscus geometry at liquid-vapour interface

It is this natural and self-regulating counteracting force that creates the pumping effect of the porous media and is known as the capillary force of the wick. The strength of this capillary pressure can be determined through the Young-Laplace equation.

$$
P_{\mathrm{c}}=P_{\mathrm{v}}-P_{\mathrm{f}}=\frac{2 \sigma}{R} \cos \theta
$$


where:
$P \quad$ is pressure
$\sigma \quad$ is surface tension
$R \quad$ is mean radius of curvature
$\theta \quad$ is wetting angle
subscript:
c designates Capillary parameter
f designates fluid parameter
v designates vapour parameter

\subsubsection{Fluid Flow in Porous Media}

In general, fluid flow through porous media can be viewed as flow through a labyrinth of conduits. Due to the high internal area of these conduits, bulk shear stress resistance dominates the flow regime [5]. In 1856, Darcy discovered that in such a flow regime, the fluid flow can be described as

$$
-\nabla P=\frac{\mu}{K} u_{\mathrm{D}}
$$

where:

$\nabla \quad$ is $\operatorname{grad}$ operator, $\left(\frac{\partial}{\partial x}, \frac{\partial}{\partial y}\right)$

$\mu \quad$ is viscosity

$K \quad$ is permeability

$u_{\mathrm{D}} \quad$ is Darcian velocity or filter velocity 
This equation is known as Darcy's Law. It can also be rewritten for anisotropic porous media using the permeability tensor.

$$
-\nabla P=\frac{\mu}{\mathbf{K}} u_{\mathbf{D}}
$$

where:

$\mathbf{K}$ is the permeability tensor, $\mathbf{K}=\left[\begin{array}{lll}K_{11} & K_{12} & K_{13} \\ K_{21} & K_{22} & K_{23} \\ K_{31} & K_{32} & K_{33}\end{array}\right]$

Since then, many have added terms to Darcy's equation to better account for different aspect of porous media flow such as boundary layers and inertial effects. Brinkman added the Brinkman term to account for effects of neighbouring spheres [5] thereby accounting for viscous penetration.

$$
\nabla P=-\frac{\mu}{K} u_{\mathrm{D}}+\mu^{\prime} \Delta u_{\mathrm{D}}
$$

where:
$\Delta \quad$ is the Laplacian operator, $\nabla \cdot \nabla$ or $\nabla^{2}$ or $\frac{\partial^{2}}{\partial x^{2}}+\frac{\partial^{2}}{\partial y^{2}}$
$\mu^{\prime} \quad$ is effective viscosity, $\mu^{\prime}=\mu[1+2.5(1-\varepsilon)]$

\section{Permeability}

Permeability, $K$, is a measure of the flow conductance in the porous media for which many empirical, semi-empirical, first principle based and numerical correlation have been developed. However, all correlations have their range of applicability and all assume a 
constant directional effective porosity meaning that the volume fraction occupied by the interconnected voids is constant in space.

\subsubsection{Thermal Transport in Porous Media}

Thermal transport in a saturated porous media strongly depends on the structure of the solid matrix and its thermal conductivity as well as the thermal conductivity of the permeating fluid [5].

\section{Effective Conductivity of Saturated Porous Media}

Though best determined by experimentation, the effective thermal conductivity of the saturated porous media can be assumed to follow one of many available correlations. These correlations have varying accuracy depending on the irregularity porous structure, the continuity in the solid matrix, the contact resistance between the solid-liquid interface and the relative magnitude of the solid matrix to that of the permeating fluid.

For granular type porous media, the leading effective conduction models were summarized by Tavman in 1996 [6] of which the first five he considered are the most widely used in literature and are summarized here.

The minimum value of effective thermal conductivity coefficient is given by a series phase configuration (solid and fluid phase in layers normal to the direction of the heat flow), which is expressed as:

$$
\lambda_{\mathrm{e}}=\frac{\lambda_{\mathrm{s}} \cdot \lambda_{\mathrm{f}}}{\varepsilon \cdot \lambda_{\mathrm{s}}+(1-\varepsilon) \cdot \lambda_{\mathrm{f}}}
$$


where:

$\lambda \quad$ is thermal conductivity coefficient

$\varepsilon \quad$ is porosity

subscript:

e designates effect porous media parameter

s designates solid parameter

When heat flow occurs parallel to solid and fluid phase layers (in parallel), then the maximum value of effective thermal conductivity is achieved which is expressed as:

$$
\lambda_{\mathrm{e}}=\varepsilon \cdot\left(\lambda_{\mathrm{f}}\right)+(1-\varepsilon) \cdot \lambda_{\mathrm{s}}
$$

All other values of effective thermal conductivity will occur within these limits. However, these limits will converge as the relative values of thermal conductivity of the solid matrix and permeating fluid is reduced. That being stated, dissimilar thermal conductivities of the solid and fluid will cause a wide range of values and therefore increase the importance of choosing the proper correlation.

The next model of importance reviewed by Tavman [6] was the weighted geometric mean model. No longer used in current literature, this correlation was used in the past as a better approximation to the limiting equations.

$$
\lambda_{\mathrm{e}}=\lambda_{\mathrm{f}}^{\varepsilon} \cdot \lambda_{\mathrm{s}}^{(1-\varepsilon)}
$$

Maxwell [7] produced a correlation that is widely used in literature for randomly distributed similar sized spheres with large interstitial distance in a homogenous medium by use of potential theory. 


$$
\lambda_{\mathrm{e}}=\lambda_{\mathrm{f}} \cdot \frac{2 \varepsilon \cdot k_{\mathrm{f}}+(3-2 \varepsilon) k_{\mathrm{s}}}{(3-\varepsilon) k_{\mathrm{f}}+\varepsilon \cdot k_{\mathrm{s}}}
$$

On the other hand, Zehner and Schlunder [8] presented a correlation for a packed bed of organized spheres with point contacts in the thermal flow direction which is also widely used in literature as well as used for this thesis.

$$
\begin{gathered}
\frac{\lambda_{\mathrm{e}}}{\lambda_{\mathrm{f}}}=(1-\sqrt{1-\varepsilon})+\frac{2 \sqrt{1-\varepsilon}}{1-\sigma B}\left[\frac{(1-\sigma) B}{(1-\sigma B)^{2}} \ln \left(\frac{1}{\sigma B}\right)-\frac{B+1}{2}-\frac{B-1}{1-\sigma B}\right] \\
B=1.25[(1-\varepsilon) / \varepsilon]^{10 / 9} \\
\sigma=\lambda_{\mathrm{f}} / \lambda_{\mathrm{s}}
\end{gathered}
$$

\section{Local Thermal Equilibrium}

To determine the temperature distribution of the saturated porous media, both the solid matrix and permeating fluid can be treated with separate transient convection-conduction equations.

$$
\begin{gathered}
(\rho C)_{\mathrm{s}} \frac{\partial T_{\mathrm{s}}}{\partial t}=\nabla \cdot \lambda_{\mathrm{s}} \nabla T_{\mathrm{s}} \\
\left(\rho C_{\mathrm{p}}\right)_{\mathrm{f}}\left[\frac{\partial T_{\mathrm{f}}}{\partial t}+u_{\mathrm{D}} \cdot \nabla T\right]=\nabla \cdot \lambda_{\mathrm{f}} \nabla T_{\mathrm{f}}
\end{gathered}
$$

where:

$\nabla \cdot \quad$ is the divergence operator

$\rho \quad$ is materials density

C is material specific heat capacity

$C_{\mathrm{p}} \quad$ is the fluids specific heat capacity at constant pressure 
$T \quad$ is temperature

$t \quad$ is time

The solid-liquid interface can be treated with continuity of temperature and heat flux.

$$
\begin{gathered}
T_{\mathrm{f}}=T_{\mathrm{s}} \\
\hat{n} \cdot \lambda_{\mathrm{f}} \nabla T_{\mathrm{f}}=\hat{n} \cdot \lambda_{\mathrm{s}} \nabla T_{\mathrm{s}}
\end{gathered}
$$

where:

$\hat{n} \quad$ is the normal unit vector

However, if effective thermal conductivity is known, the temperature distribution can be determined with the local volume-averaged thermal equilibrium model [5], see Equation (2.14). This macroscopic approach will not allow for differentiating between the solid matrix and the permeating fluid in the analysis.

$$
\left[\varepsilon\left(\rho C_{\mathrm{P}}\right)_{\mathrm{f}}+(1-\varepsilon)(\rho C)_{\mathrm{s}}\right] \frac{\partial\langle T\rangle}{\partial t}+\left(\rho C_{\mathrm{P}}\right)_{\mathrm{f}} u_{\mathrm{D}} \cdot \nabla\langle T\rangle=\nabla \cdot \lambda_{\mathrm{e}} \nabla\langle T\rangle
$$

where:

\langle\rangle indicates local averaged value

\section{Local Thermal Non-Equilibrium}

If the average pore size is in the same order of magnitude as the domain size to be analysed, then it is best to treat the analysis as a direct simulation where no assumptions are made about the local thermal equilibrium. For increased accuracy, this approach can also be taken if the difference in thermal conductivity coefficient between the solid matrix and the permeating fluid is very large in a highly convective flow as shown by Jiang et al. [9]. 
One method of local thermal non-equilibrium is that of Sahraoui and Kaviany [10] who developed an interfacial temperature slip boundary condition to be used instead of continuity of temperature and heat flux (Equation (2.12) and Equation (2.13)) [5].

$$
\left.\frac{d\langle T\rangle_{V}}{d y}\right|_{y=0^{-}}=\frac{\alpha_{T}}{\lambda}\left(T^{-}-T^{+}\right)
$$

where

$\alpha_{T} \quad$ is the dimensionless slip coefficient

$T^{-} \quad$ is the solid medium interfacial temperature

$T^{+} \quad$ is the fluid medium interfacial temperature

$\pi \quad$ is the pore length scale.

\subsection{Loop Heat Pipe Evaporator Models}

There are three basic phenomena that occur within the loop heat pipe's evaporator that if simulated properly, can model any speculated internal scenarios at any applied heat flux. However, all scenarios are dependent on the ability to accurately model the first phenomena which is saturation of the wick demonstrated in Figure 2.3-a. The next two phenomena are much more difficult in their modelling implementation. When operating at low heat fluxes, excess liquid can accumulate in the vapour groove localized where the fin meets the wick [11]. This accumulate will form a meniscus as demonstrated in Figure 2.3-b due to surface tension and pressure difference across the liquid-vapour interface and will reduce in size as the applied heat flux increases. The modelling of the last phenomena which is beyond the scope of this thesis deals with the development of a 
vapour pocket at the fin-wick interface. This vapour pocket will be centralized against the heating fin $[11][12]$ and can be either trapped under the heating fin as demonstrated in Figure 2.3-c or venting to the vapour groove as demonstrated in Figure 2.3-d. In both cases, the thermal resistance of the fin-wick contact is increased. It can also be conceived that a scenario where the meniscus and a trapped vapour pocket coexist (not illustrated in Figure 2.3) [11].

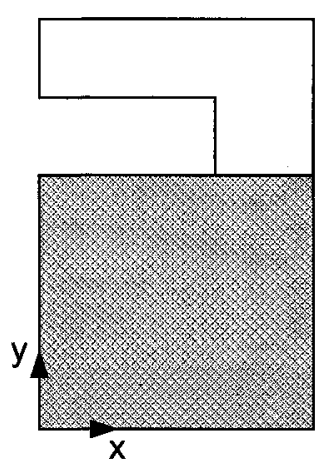

a)

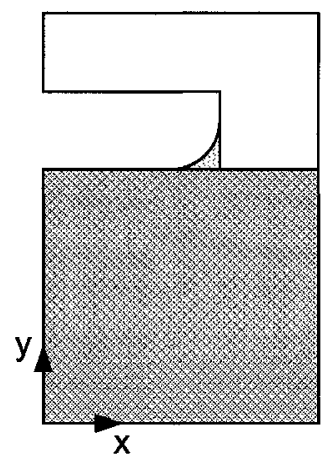

b)

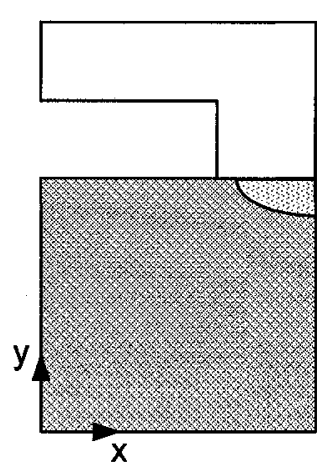

c)

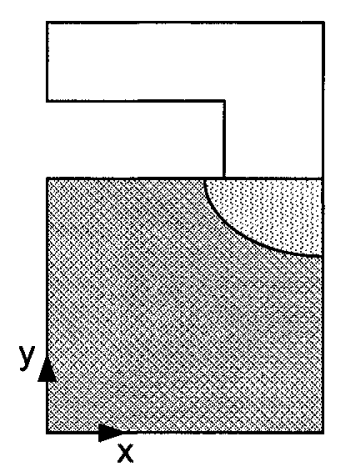

d)

Figure 2.3 Basic phenomena occurring in loop heat pipe operation: a) Saturation of wick, b) Access fluid accumulating in vapour groove in the form of a meniscus, c) Trapped vapour pocket and d) Vapour pocket reaching vapour groove

The greatest difficulty in implementing the last two phenomena (presence of meniscus and vapour pocket) in a model is that their geometry is unknown. This creates a need for an extensive iterative process for boundary locating that may not converge. As such, the numerical modelling of the complete evaporator involving the complexity of the heat and mass transfer process in the wick, vapour and casing is quite difficult. 
Of all the existing published models examined, none were applicable in all operational regimes. Furthermore, a variety of simplifying assumptions were made to render the solution attainable and less computationally tedious.

Summarized in upcoming subsections are pertinent reported evaporator models that can be categorized into different levels of completeness:

1. Models that simulate the wick only;

2. Models that simulate the wick and part or all of the casing;

3. Models that simulate the wick, the casing, and compensate for the vapour.

In addition, evaporator models may be subcategorized into the following internal scenarios:

a. Meniscus present in vapour groove;

b. Fully saturated wick;

c. Vapour pocket under heated fin;

d. Vapour pocket reaching the vapour groove;

e. Coexistence of meniscus and the vapour pocket.

\subsubsection{Evaporator model of Domidov and Yatsenko (1994) [8]}

Domidov and Yatsenko [11] numerically investigated a flat type micro evaporator (200 $\mu \mathrm{m}^{2}$ thick wick) with a $2 \mathrm{D}$ steady state model that analyzed the wick and a portion of the casing. They demonstrated that the casing was an integral part of the model because the thermal flux undergoes curvature as it enters the wick to reach the evaporative surface. The internal scenarios investigated by Domidov and Yatsenko [11] were that of the vapour pocket and are, to the best of the author's knowledge, the only ones to have 
qualitatively investigated the meniscus thus categorizing this model as a 2 -a,d,e. Emphasis in their investigation was given to the origin and growth of vapour pocket as well as the evaporation intensity distribution.

When developing their model, Demidov and Yatsenko [11] included both convection and diffusion within the wick. To approximate the location of the liquid-vapour interface within the wick (locating the vapour pocket), the heat drain, mass flux, and capillary pressure jump equations were used and a single value for thermal conduction was applied both the vapour pocket and saturated wick region. The meniscus, however, was not numerically modelled. An analytical approach that consisted of applying a localized increase in the convection rate on the evaporative surface was used to qualitatively simulate the presence of the meniscus. This approach neglects the meniscus' internal flow.

From their investigation, Domidov and Yatsenko [11] concluded that a stable vapour pocket can exist and that it would grow in size when the applied heat load is increased starting from a point at the center of the heating fin. Additionally, Domidov and Yatsenko [11] concluded that in the presence of the meniscus the evaporation of its surface would exceed that of the wicks surface by two to three times.

\subsubsection{Evaporator model of Cao and Faghri (1994) [12]}

Cao and Faghri [12] mathematically formulated a transient model for the flat type evaporator incorporating the analysis of a $3 \mathrm{~mm}$ thick wick, the casing, and a 3D vapour flow in the groove in order to conduct a performance analysis of multiple working fluids. As such, this model is categorized as a 3-b,d. 
This model seems to be the most complex. It includes both convection and diffusion terms as part of the wick's governing equations, the transient Navier Stokes equations were used in the vapour groove, and the casing was solved using pure conduction. These were solved in an uncoupled fashion using an iterative procedure to converge the energy and mass conservation equations at each time step as a conjugate problem.

Cao and Faghri [12] concluded that boiling at the fin-wick interface is a major concern and can be an operational limitation. Most importantly, they also concluded that water vapour may experience moderately high velocities in the vapour groove, whereas ammonia and Freon-11 vapour flow velocities where insignificant leading to reasonably accurate results if modeled in $2 \mathrm{D}$ and neglecting the vapour groove.

\subsubsection{Evaporator model of Khrustalev and Faghri (1995) [13]}

Khrustalev and Faghri [13] published a model for an alternative evaporator configuration that used a triangular fin penetrating the wick as shown in Figure 2.4. They developed a one-dimensional mathematical model by means of thin film evaporation theory and heat transfer in porous media. In a manner different then the two models discussed previously, Khrustalev and Faghri [13] used Kelvin's equation to determine the vapour's saturation pressure at the liquid-vapour interface within the wick. Most importantly, Khrustalev and Faghri [13] concluded that the thermal resistance would increase along with the applied thermal flux, though this resistance could be lowered through the use of a wick with a larger thermal conductivity. 


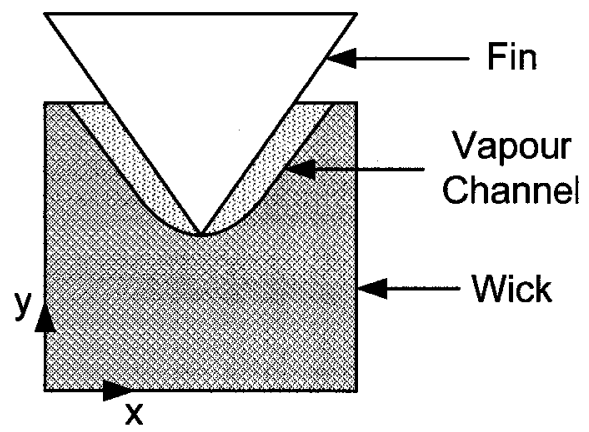

Figure 2.4 Evaporator studied by Khrustalev and Faghri [13]

\subsubsection{Evaporator model of Figus et al. (1999) [14]}

Figus et al. [14] published an evaporator model that numerically investigated the flat type evaporator with a 2D, steady state model that neglected all components with the exception of the $5 \mathrm{~mm}$ thick wick. Although thermal convection was neglected within the wick, Figus et al. [14] modelled the fluid flow with two sub-models for comparison; a continuum Darcy model, and a pore network model. The range of the applied heat flux was also restricted to the amount that would cause the vapour pocket to be vented to the vapour groove with an overall wick pressure drop of $40 \mathrm{~Pa}$. This model is categorized as a $1-d$.

Figus et al. [14] established that the Darcian fluid flow sub-model could accurately predict the vapour pocket shape when a single pore size is considered. They also concluded that by varying the pore size distribution (which may restrict the performance of the evaporator) the pore network model predicts an irregular vapour pocket shape that cannot be seen using the Darcy model. 


\subsubsection{Evaporator model of Kaya and Goldak (2006) [15]}

Kaya and Goldak [15] numerically investigated the tubular evaporator with a 2D steady state model containing the $7 \mathrm{~mm}$ thick wick, the full casing and the vapour values of pressure and saturation temperature as determined by a separate $1 \mathrm{D}$ model reported in [3]. This evaporator model coupled the thermal convection model to the Darcy model within the wick through the use of an intensive iterative procedure. Both the fully liquid saturated wick and the presence of a vapour pocket were investigated, thus categorizing this model as a 3-b,d. In the latter scenario (the vapour pocket), temperature conservation and the heat drain equations were used with an additional iterative process to predict the pocket's shape. The superheat limits within the wick were estimated using cluster nucleation theory, whereas the superheat in the vapour groove was prescribed at 3 degrees superheat.

Kaya and Goldak [15] concluded that the existence of the vapour pocket is feasible and probable. However, they established that the wick does not experience dry outs as a result of high applied heat flux and that boiling was very unlikely at the vapour pocket liquid-vapour interface.

\subsubsection{Evaporator model of Ren et al. (2007) [16]}

Ren et al. [16] published their numerical investigation of a tubular evaporator with a 2D transient model containing only a $5 \mathrm{~mm}$ thick wick and excluding other components. The governing equation for the transient fluid flow within the wick was derived using the transient continuum equation, Darcy's equation, and the equation of state thus allowing for slight compressibility within the fluid. In a manner similar to the Khrustalev and 
Faghri's model [13], Kelvin's equation was used to determine the vapour's saturation pressure within the wick. An aspect that sets this paper apart from others is the assumption that a thin vapour film exists at the fin-wick interface during low applied heat flux operation. Furthermore, Ren et al. [16] also set both the incoming pressure and the pressure at the evaporative surface to the same value. As a result of the combination of these two assumptions, the pressure and velocity results published by Ren et al. [16] differ significantly from all others.

Ren et al. [16] concluded that the vapour pocket penetrates deeper into the wick when evaporation and thermal conduction is stronger than the convective cooling capabilities of the incoming fluid. Ren et al. [16] also observed that due to convection, an increase in evaporation will reduce the overall temperature of the wick by increasing the incoming mass flow rate of the cooler liquid, thereby demonstrating the need to include thermal convection in the considerations.

\subsubsection{Literary Trends and Conclusions}

As seen in the six models summarized, all six investigate the vapour pocket and only one analytically investigates the possibility of the meniscus. Individual contributions include: the acceptance of the vapour pocket's existence and demonstration of results from possible meniscus; results demonstrating that a $2 \mathrm{D}$ model neglecting vapour flow can give accurate results in some cases; a validation of the use of Darcy's Equation when modelling a single pore size; confidence that wick dry outs are very rare as well as boiling at the vapour pockets interface; and a general observation that convection will cool the wick. Common trends throughout literature demonstrate that a vapour pocket 
will form at the center of the heating fin when the liquid within the wick reaches a critical superheat temperature, although some disagreement was observed in the method used to determine its value. However, this vapour pocket seems to be reserved for moderately to high fluxes. Low heat flux investigation appears to have been relatively neglected by the literature. Though the loop heat pipe is a high efficiency thermal transport device that is primarily used in applications where there is a high risk of excessive heat loads, it is also possible that during startup or idle operation of certain applications that low heat loads can be considered. Currently, to the best of the author's knowledge, the evaporator model developed for this thesis is the first model that numerically solves the evaporator domain while also numerically considering the presence of the meniscus. 


\section{Chapter 3: Computational Model Formulation}

When modelling the evaporator, the tubular or flat plate evaporator's cross section contain planes of symmetry that can be used to divide the numerical domain into smaller sections which will reduce the computational requirements without compromising the quality and accuracy of the results, see Figure 3.1 .

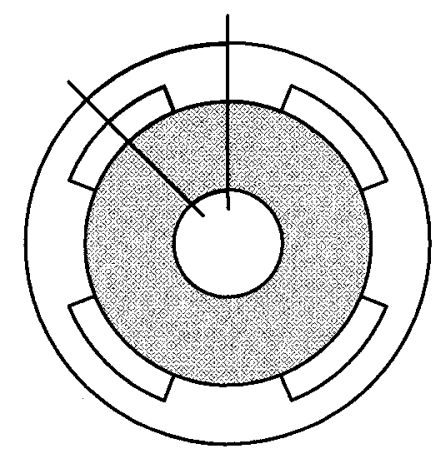

a)

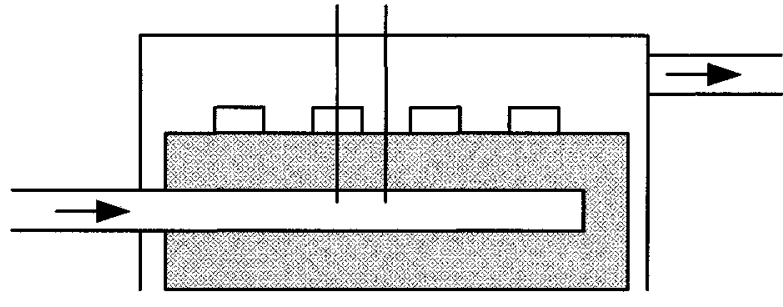

b)

Figure 3.1 Evaporator cross-sectional cut demonstrating computational domain: a) Tubular evaporator, b) Flat Plate evaporator

If the tubular evaporator were chosen, the Polar coordinates would be best suited for its evaluation. The Cartesian coordinates is well suited for the flat type evaporator. Seeing as the tubular evaporator domain can be approximated by a flat type evaporator with similar radial differences for ordinate dimensions and a mean width for the abscissa or can be directly simulated using the following coordinate transformation: 


$$
\begin{aligned}
& r=\sqrt{x^{2}+y^{2}} \\
& \theta=\tan ^{-1}\left(\frac{y}{x}\right)
\end{aligned}
$$

for Cartesian to Polar coordinates or

$$
\begin{aligned}
& x=r \cos \theta \\
& y=r \sin \theta
\end{aligned}
$$

for Polar to Cartesian coordinates

where:

$r \quad$ is the radial coordinate

$\theta \quad$ is the angular coordinate or polar angle

$x \quad$ is the abscissa

$y \quad$ is the ordinate

and that the use of Cartesian coordinates simplifies the governing equations as well as their solution, this thesis was entirely concluded in Cartesian coordinates simulating a flat plate evaporator.

Furthermore, some of the simplifications adopted by others as described in Chapter 2: were also adopted for this thesis to reduce the model complexity and render its development feasible. The first major simplification is that the vapour in the groove is not modeled since a computationally intensive full 3D Navier Stokes model is necessary to account for the axial addition and removal of mass at the evaporative surface. Furthermore, Cao [12] demonstrated that reasonable accuracy can be obtained without the vapour model especially when the vapour velocities are small, which is expected at 
low applied heat fluxes. However, to compensate for lack of numerical vapour groove results, a 1D mathematical loop heat model presented by Kaya and Hoang [3] was used to produce vapour pressure and saturation temperature results as a function of applied flux for boundary conditions as was done in [15]. Secondly, the boundaries are kept stationary on the wick. As such, the vapour remains outside of the computational domain. This latter restriction inhibits the modelling of boiling within the porous media and excessive heat input which would cause the liquid-vapour boundary to recess into the porous media. Thirdly, the wick was considered to be homogeneous and isotropic which simplified the governing equations by reducing their coefficients to a constant instead of a directionally sensitive tensor. When considering the porous media and the flow within it, Vafai and Tien investigated the importance of the boundary and inertial effects on the flow and heat transfer in porous media and have determined that they often have no significant effect on the overall flow field [17]. As such, the fourth simplification comprises of excluding the non-Darcian terms in the fluid flow governing equation. It was also assumed that the thermal field within the saturated porous wick is governed by local thermal equilibrium thereby enabling the use of a volume averaged macroscopic analysis. The effects of gravity were also considered to be negligible due to short path length of liquid traveling against gravity within the porous media. Other important assumptions included no radiation and constant material properties.

The two scenarios modelled in this thesis are presented in Figure 3.2 (the domain numbering and the perimeter location designation as illustrated in Figure 3.2 will be used for the remainder of this thesis). The first scenario (Figure 3.2-a) is that of a saturated 
wick and the casing which models the desired design operating condition. The second scenario (Figure 3.2-b) is the same as that of the first with the addition of excess fluid appearing in the vapour groove in the form of a meniscus as a result of low heat fluxes. The evaporator model developed to simulate both of these scenarios consists of four submodels and was programmed to determine pressure, velocity, and temperature profiles.

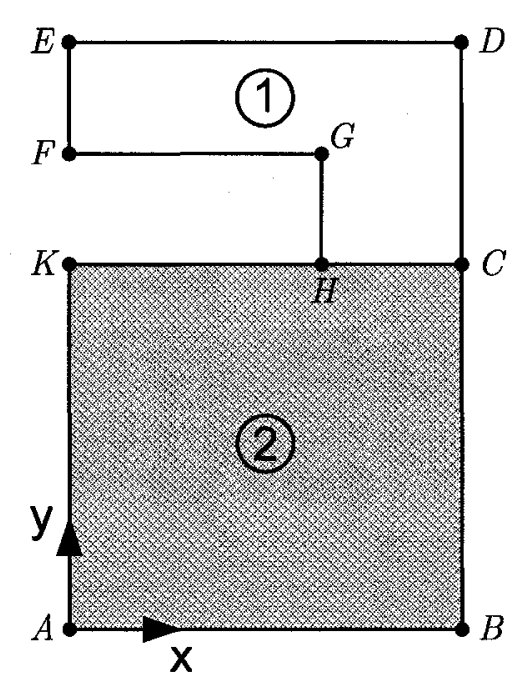

a)

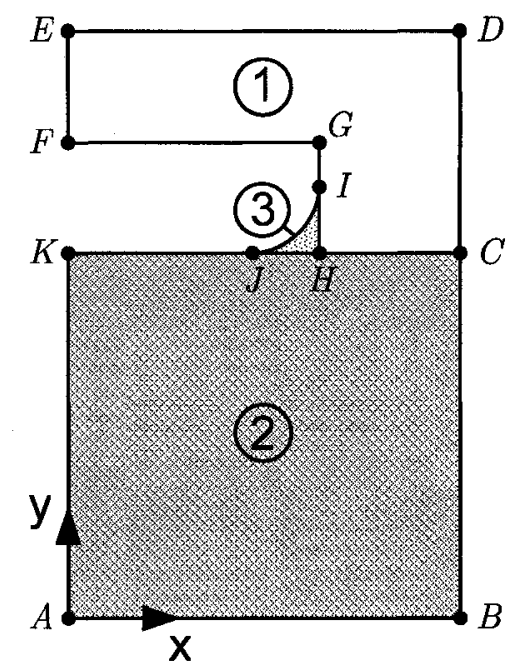

b)

Figure 3.2 Computational domains: a) Design operating condition, b) Low heat flux condition

\subsection{Diffusion Thermal Sub-Model}

The pure diffusion thermal sub-model is used to solve thermal conduction in the casing (component - 1) and the meniscus (component - 3) as well as a preliminary guess for the wick (component - 2).

\subsubsection{Equations}

The Eulerian equation for thermal diffusion is 


$$
\rho C \frac{\partial T}{\partial t}=\nabla \cdot \lambda \nabla T
$$

When this equation is used in the wick domain, the thermal conductivity coefficient, $\lambda$, is replaced with the effective thermal conductivity coefficient, $\lambda_{\mathrm{e}}$. Also, at steady state, Equation (3.1) is reduced to

$$
\nabla \cdot \lambda \nabla T=0 \text { or } \lambda \Delta T=0
$$

\subsubsection{Boundary Conditions}

Initially both the casing and the wick are modelled using the thermal diffusion model and are solved simultaneously. As such, at the boundary $\mathrm{HC}$, between the wick and the casing, both conservation of temperature and flux exist.

$$
\begin{gathered}
T_{\mathrm{c}}=T_{\mathrm{w}} \\
\lambda_{\mathrm{c}} \nabla T_{\mathrm{c}}=\lambda_{\mathrm{e}} \nabla T_{\mathrm{w}}
\end{gathered}
$$

where subscript:

c designates casing parameter

w designates wick parameter

The saturation temperature is prescribed as a Dirichlet temperature boundary condition at the fluid entrance, boundary $\mathrm{AB}$

$$
T=T_{\text {sat }}
$$

and the vapour temperature is prescribed at the evaporation surface, boundary $\mathrm{KH}$

$$
T=T_{\text {vap }}
$$


where:

$T_{\text {sat }} \quad$ is saturation temperature

$T_{\text {vap }} \quad$ is vapour temperature

On all vertical plane of symmetry, boundary $\mathrm{AK}, \mathrm{FE}, \mathrm{BC}$ and $\mathrm{CD}$, a Neumann boundary condition is prescribed

$$
q_{\mathrm{x}}=-\lambda \frac{\partial T}{\partial x}=0
$$

where:

$q_{\mathrm{x}} \quad$ is $\mathrm{x}$-component of thermal flux

On the interface between the vapour groove and the casing, boundary FG and GH, a convective heat loss is applied to simulate the casing superheating the vapour.

$$
\lambda \frac{\partial T}{\partial \hat{n}}=\alpha \cdot\left(T-T_{v a p}\right)
$$

where:

$\alpha \quad$ is thermal convective coefficient

The heat flux applied is a Neumann boundary condition prescribed to the top surface of the casing, boundary ED.

$$
q_{\mathrm{y}}=-\lambda \frac{\partial T}{\partial y}=q_{\mathrm{in}}
$$

where:

$q_{\mathrm{y}} \quad$ is y-component of thermal flux

$q_{\text {in }} \quad$ is thermal flux applied to casing 


\subsection{Convection-Diffusion Thermal Sub-Model}

The convection-diffusion thermal sub-model is used to solve the thermal field in the wick (component - 2) only. Here, a macroscopic volume-averaged approach with local thermal equilibrium is used. Due to the lower convection rates of the fluid in the loop heat pipe, especially in the range of heat fluxes investigated, the accuracy gained by a local non-equilibrium thermal model would be minimal and therefore does not warrant the greater difficulties of its implementation.

\subsubsection{Equations}

The Eulerian equation for thermal convection-diffusion is

$$
\left[\varepsilon\left(\rho C_{\mathrm{P}}\right)_{\mathrm{f}}+(1-\varepsilon)(\rho C)_{\mathrm{s}}\right] \frac{\partial\langle T\rangle}{\partial t}+\left(\rho C_{\mathrm{P}}\right)_{\mathrm{f}} u_{\mathrm{D}} \cdot \nabla\langle T\rangle=\nabla \cdot \lambda_{\mathrm{e}} \nabla\langle T\rangle
$$

The first term on the left hand side is the transient term with a volume-averaged thermal capacitance and the following term is the convective term. The right hand side is occupied by the conduction term. Again, since the steady state result is required, Equation (3.10) is reduced to

$$
\left(\rho C_{\mathrm{P}}\right)_{\mathrm{f}} u_{\mathrm{D}} \cdot \nabla\langle T\rangle=\nabla \cdot \lambda_{\mathrm{e}} \nabla\langle T\rangle
$$

The triangular brackets on the temperature variable represent the volume averaged value and should be carried through however for ease of explanation and since it is assumed that $\langle T\rangle=T$ in this case, the brackets will be dropped from this point on. 


\subsubsection{Boundary Conditions}

When the wick is modelled using the thermal convection-diffusion model, the casing is solved using the thermal diffusion model and both models are solved simultaneously. Once more, conservation of temperature and flux exist at the interface between the wick and the casing, boundary $\mathrm{HC}$.

$$
\begin{gathered}
T_{\mathrm{c}}=T_{\mathrm{w}} \\
\lambda_{\mathrm{c}} \nabla T_{\mathrm{c}}=\lambda_{\mathrm{e}} \nabla T_{\mathrm{w}}
\end{gathered}
$$

All other boundary conditions remain the same as that described in Section 3.1.2. Dirichlet temperature boundary conditions are prescribed at the fluid entrance, boundary $\mathrm{AB}$, and the evaporation surface, boundary $\mathrm{KH}$ (as demonstrated in Equation (3.5) and Equation (3.6) respectively). A Neumann boundary condition is prescribed on the vertical plane of symmetry, boundary AK and BC (as demonstrated in Equation (3.7)).

\subsection{Laplacian Pressure Sub-Model}

The fluid flow is only considered in the wick (component - 2).

\subsubsection{Equations}

Since the wick is considered fully saturated (no boiling) at all times and the working fluid is considered incompressible, conservation of mass dictates that the divergence of the velocity is zero

$$
\nabla \cdot u_{\mathrm{D}}=0
$$

Furthermore, since we are working with fluid flow within a porous media, the velocity field is dictated by Darcy's Law 


$$
-\nabla P=\frac{\mu}{K} u_{\mathrm{D}}
$$

By combining Equation (3.14) and Equation (3.15), the Laplace equation for pressure is obtained and is solved to determine pressure field throughout the wick.

$$
\Delta P=0
$$

\subsubsection{Boundary Conditions}

The corresponding saturation pressure to the imposed saturation temperature of the liquid entering the porous media is prescribed as a Dirichlet pressure boundary condition at the fluid entrance, boundary AB.

$$
P=P\left(T_{\text {sat }}\right)
$$

The normal velocity at the wick-fin interface, boundary $\mathrm{HC}$, must be zero. Using Darcy's Law, Equation (3.15) and the known normal velocity the Neumann pressure boundary condition is derived.

$$
\frac{\partial P}{\partial y}=0
$$

The symmetry Neumann boundary condition is prescribed on the vertical planes, boundary AK and BC.

$$
\frac{\partial P}{\partial x}=0
$$

At the liquid-vapour interface, boundary $\mathrm{KH}$, the liquid velocity can be determined from the evaporation mass flow rate which is a function of surface normal temperature gradient 


$$
\frac{\dot{m}}{A}=\frac{q_{\mathrm{y}}}{h_{\mathrm{fg}}}=-\frac{\lambda_{\mathrm{e}}}{h_{\mathrm{fg}}} \frac{\partial T}{\partial y}
$$

where:

$A \quad$ is surface area

$h_{\mathrm{fg}} \quad$ is latent heat of evaporation

and from the mass flow rate equation

$$
\frac{\dot{m}}{A}=\rho u
$$

Combining Equation (3.20) and Equation (3.21), the liquid velocity at boundary KH is

$$
u_{D}=\frac{\lambda_{\mathrm{e}}}{\rho h_{\mathrm{fg}}} \frac{\partial T}{\partial y}
$$

Combining Equation (3.22) with Darcy's Law, Equation (3.15), the Neumann boundary condition is obtained.

$$
\frac{\partial P}{\partial y}=-\frac{\mu}{K} \frac{\lambda_{\mathrm{e}}}{\rho h_{\mathrm{fg}}} \frac{\partial T}{\partial y}
$$

\subsection{Darcian Velocity Sub-Model}

Once the pressure field has been determined, Darcy's Law, Equation (3.15), is used to convert pressure gradients into velocity fields.

$$
\begin{aligned}
& \frac{\partial P}{\partial x}=-\frac{\mu}{K} u_{\mathrm{DX}} \\
& \frac{\partial P}{\partial y}=-\frac{\mu}{K} u_{\mathrm{DY}}
\end{aligned}
$$


where:

$u_{\mathrm{DX}} \quad$ is X-component of velocity

$u_{\mathrm{DY}} \quad$ is $\mathrm{y}$-component of velocity

\subsection{Addition of Fluid Meniscus}

To simulate the second scenario, the addition of excess fluid in the vapour groove (the meniscus, component - 3) must be implemented as a supplementary numerical domain. This requires that the model be capable of predicting the shape of the meniscus as well as solving the extra accompanying governing equation and boundary conditions.

\subsubsection{Domain Shape}

To obtain the shape of the fluid meniscus, the Young-Laplace equation (Equation (2.1)) has been reorganized to isolate the meniscus' radii as a function of vapour pressure, and liquid pressure where the fin meets with wick.

$$
R=\frac{2 \sigma}{P_{\text {cap }}} \cdot \cos \theta=\frac{2 \sigma}{P_{\infty}-P_{\mathrm{H}}} \cdot \cos \theta
$$

where:

$P_{\mathrm{H}} \quad$ is pressure at point $\mathrm{H}$ (point where fin meets wick)

$P_{\infty} \quad$ is vapour pressure leaving the wick

Since the physical wetting angle is unknown, this thesis will assume an angle of zero which represents the maximum possible capillary pressure as done in [11]. Therefore, Equation (3.26) will be reduced to 


$$
R=\frac{2 \sigma}{P_{\infty}-P_{\mathrm{H}}}
$$

\subsubsection{Boundary Condition Alterations}

In the thermal analysis, the meniscus is modeled using the thermal diffusion model as a simplifying assumption since the internal velocity field is unknown. Although solving the Navier-Stokes equations is the correct approach to determine velocity field within this domain, computational limitations and presumed negligible deviation from accuracy due to small domain size as well as low velocity led to this simplification; this simplification is implemented in [11]. However, if convection were taken into account, the meniscus temperature would slightly increase resulting in an increase in local evaporation rate while reducing its size therefore causing little change to the overall loop heat pipe operating response.

The thermal analysis of all domains was resolved simultaneously to conserve temperature and thermal flux at the fin-meniscus interface, boundary HI and the wickmeniscus interface, boundary $\mathrm{JH}$.

Boundary HI

$$
\begin{gathered}
T_{\mathrm{c}}=T_{\mathrm{m}} \\
\lambda_{\mathrm{c}} \nabla T_{\mathrm{c}}=\lambda_{\mathrm{m}} \nabla T_{\mathrm{m}}
\end{gathered}
$$

Boundary JH

$$
\begin{gathered}
T_{\mathrm{w}}=T_{\mathrm{m}} \\
\lambda_{\mathrm{e}} \nabla T_{\mathrm{w}}=\lambda_{\mathrm{m}} \nabla T_{\mathrm{m}}
\end{gathered}
$$


where subscript:

m designates meniscus parameter

At the evaporative surface of the meniscus, boundary $\mathrm{JI}$, the vapour temperature is prescribed.

$$
T=T_{\text {vap }}
$$

Though thermal diffusion is the only result obtained within the meniscus, its presence is also felt in the pressure model. When calculating the pressure field in the wick, the evaporation will remain unchanged on boundary KJ. However, evaporation no longer occurs directly on boundary $\mathrm{JH}$ with the meniscus present. To relate the evaporation rate that occurs on the meniscus surface, boundary JI, to the basal location of the meniscus (the surface of the wick underneath the meniscus), boundary $\mathrm{JH}$, conservation of mass is used

$$
\left.\dot{m}\right|_{\mathrm{JI}}=\left.\dot{m}\right|_{\mathrm{JH}}
$$

and rearranged to derive a relationship of the meniscus surface velocity to its basal velocity

$$
\left.u\right|_{\mathrm{JH}}=\left.\frac{\left.A\right|_{\mathrm{JI}}}{\left.A\right|_{\mathrm{JH}}} \cdot u\right|_{\mathrm{JI}}
$$

As seen in Equation (3.34) and demonstrated in Figure 3.3, due the geometry of the meniscus, the area relationship causes the average surface liquid velocity to be 1.57 times greater at the basal location. 


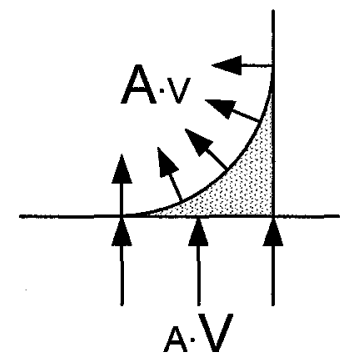

Figure 3.3 Mass conservation within meniscus

Similarly to Equation (3.20), the evaporation mass flow rate is dictated by the normal temperature gradient at the meniscus surface; here however the normal derivative cannot be simplified to the y-derivative.

$$
\dot{m}=-\left.\left.A\right|_{\mathrm{JI}} \frac{\lambda}{h_{\mathrm{fg}}} \frac{\partial T}{\partial \hat{n}}\right|_{\mathrm{JI}}=\left.\left.A\right|_{\mathrm{JI}} \cdot \rho \cdot u\right|_{\mathrm{JI}}
$$

Combining Equation (3.34) and Equation (3.35), a function for basal velocity in relation to normal surface temperature gradient is achieved

$$
\left.u\right|_{\mathrm{JH}}=\frac{-1}{\left.A\right|_{\mathrm{JH}}} \frac{\lambda}{\rho \cdot h_{\mathrm{fg}}}\left(\left.\left.A\right|_{\mathrm{JI}} \frac{\partial T}{\partial \hat{n}}\right|_{\mathrm{JI}}\right)_{\mathrm{AVG}}
$$

As done previously, Darcy's Law, Equation (3.15), is used to convert the liquid velocity to a Neumann pressure boundary condition

$$
\left.\frac{\partial P}{\partial \hat{n}}\right|_{\mathrm{JH}}=\frac{1}{\left.A\right|_{\mathrm{JH}}} \frac{\mu}{\rho \cdot K} \frac{\lambda}{h_{\mathrm{fg}}}\left(\left.\left.A\right|_{\mathrm{J}} \frac{\partial T}{\partial \hat{n}}\right|_{\mathrm{J}}\right)_{\mathrm{AVG}}
$$

The normal temperature gradients are straightforward to calculate as long as they are aligned with the abscissa or ordinate. However, along the meniscus, section JI, where the interface is curvilinear the calculation is much more involved. Since the x-component and y-component of the gradient are simple to calculate, the projection along the normal 
can then be determined knowing a few variables and using similar triangle theory as demonstrated in Figure 3.4.

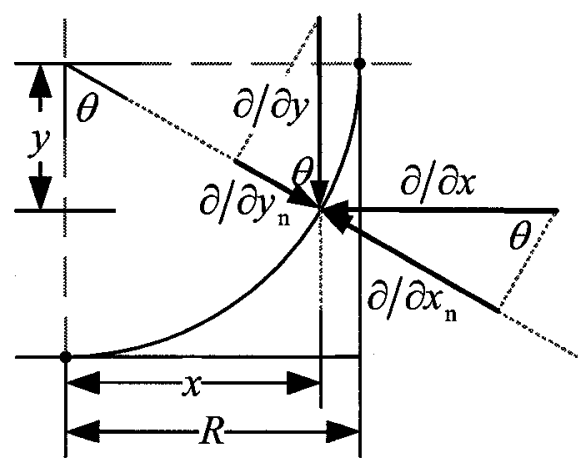

Figure 3.4 Surface normal gradient calculation schematic

The projection of the y-component of the gradient on the surface normal is

$$
\cos \theta=\frac{y}{R}=\frac{\partial y_{\mathrm{n}}}{\partial y} \Rightarrow \partial y_{\mathrm{n}}=\frac{y \cdot \partial y}{R}
$$

where:

$\theta \quad$ is the angle to the vertical axis

$\partial y \quad$ is the $y$-component of gradient

$\partial y_{n} \quad$ is the projection of the $y$-component of gradient

Similarly for the normal projection of the $\mathrm{x}$-component is

$$
\sin \theta=\frac{x}{R}=\frac{\partial x_{\mathrm{n}}}{\partial x} \Rightarrow \partial x_{\mathrm{n}}=\frac{x \cdot \partial x}{R}
$$

where:

$\partial x \quad$ is the $\mathrm{x}$-component of gradient

$\partial x_{\mathrm{n}} \quad$ is the projection of the $\mathrm{x}$-component of gradient 
The normal gradient of temperature on the meniscus surface is the summation of the projected $\mathrm{x}$ - and $\mathrm{y}$-components of thermal gradient onto the normal vector. Taking in consideration the direction of the normal in the investigated geometry, this results to

$$
\frac{\partial T}{\partial \hat{n}}=\left.\frac{\partial T}{\partial y}\right|_{\hat{\mathfrak{n}}}-\left.\frac{\partial T}{\partial x}\right|_{\hat{\mathrm{n}}}
$$

\subsection{Method of Solution}

The steady state $2 \mathrm{D}$ evaporator model developed here solves the diffusive thermal equation in the casing and meniscus, the convective-diffusive thermal equation, the Laplace equation and Darcy's flow equation in the wick. As described in Section 3.1-3.5, the energy conservation condition couples the domains at their respective interfaces. To couple the fluid flow to the convective-diffusive thermal equation, an iterative procedure is necessary. The iterative procedure selected increased the computational time and storage requirements but decreased the complexity of programming. Furthermore, within the sub-models, a Finite Element Method (FEM) solver was used to solve the governing equations with their appropriate boundary conditions.

\subsubsection{FEM Solver}

Several requirements are necessary in choosing an appropriate Finite Element Method solver: the ability to generate a mesh with multiple domains, the ability to discretize governing equations and the ability to independently define suitable boundary conditions. Given that the commercially available code MATLAB 7.0.1 has a built in partial differential equation toolbox, the PDETool, capable of creating a mesh consisting of 3- 
node triangles that approximates the domain with piecewise linear continuous basis functions as well as contains a non-linear solver using a damped Newton iteration with the Armijo-Goldstein line search strategy, this toolbox satisfies most of the requirements and therefore was used as a base for the evaporator model. Further benefits exist in using the MATLAB code: capabilities with matrix manipulation within the Finite Element algorithms as it is fundamentally a matrix solver based software, built-in functions for data visualization as well as a wide array of numerical algorithms for result analysis, and it is modular therefore can be integrated with existing and upcoming MATLAB models of other loop heat pipe components to complete the loop heat pipe model if needed.

The PDETool discretizes the diffusive elliptic equation, Equation (3.41), as well as the Dirichlet and Neumann boundary conditions, Equation (3.42) and (3.43) respectively. Subsequently, the discretized elliptic equation can be reformulated into a linear system as typically done in Finite Element theory.

$$
\begin{gathered}
-\nabla \cdot(c \nabla u)+a u=f \\
h \cdot u=r \\
\hat{n} \cdot c \cdot \nabla u+q \cdot u=g
\end{gathered}
$$

where:

$u \quad$ is the unknown solution (PDETool)

c is the conductive coefficient (PDETool)

$a, f \quad$ are the elliptic equation coefficients (PDETool)

$h, r$ are the Dirichlet boundary condition coefficients (PDETool)

$q, g$ are the Neumann boundary condition coefficients (PDETool) 


\subsubsection{PDETool Workarounds}

Though the availability of the MATLAB PDETool has greatly helped in the development of the sub-models described above, Section 3.1 through 3.4 , it was a base code that required many workarounds to enable its use for the development of the evaporator model. The major workarounds are presented in the following subsections.

\section{Lack of Communication}

The greatest challenge to the use of the PDETool, is that it does not communicate directly with the command line and therefore does not recognise all variables introduced into the MATLAB workspace. This is not a difficulty if the computational problem being solved is directly realizable in a single iteration. However, since the evaporator model requires a solution to equations where temperature and pressure are implicitly coupled, iteration between the governing equations and boundary conditions is problematic.

To ensure sufficient convergence, the functions for pressure and temperature that were separately created and independently solved using the algorithms within the PDETool box, were at each iteration updated with values being passed into the next loop. This passing of information required the creation of multiple supplementary functions that used text-based write/read information to circumvent the lack of communication. Nevertheless, the implementation of these functions rendered the code more modular which had the benefit of easier organization and debugging. 


\section{Convective Term}

Another great challenge with the PDETool was the solution of the convection term in the thermal governing equation for the wick. The PDETool is not equipped to solve convection terms with first order space derivatives [18]. Therefore, a sub-routine was written to use a known velocity field from the previous iteration (through the use of functions as described in Lack of Communication Section), multiplying them by their respective thermal first order space derivative of temperature, see Equation (3.44), and applying the product to the $f$ coefficient of Equation (3.41). These values were updated at every internal iteration and could only be solved using the non-linear solver.

$$
f=\left(\rho C_{\mathrm{P}}\right)_{\mathrm{f}} u_{\mathrm{D}} \cdot \nabla T=\left(\rho C_{\mathrm{P}}\right)_{\mathrm{f}}\left[u_{\mathrm{DX}} \cdot \frac{\partial T}{\partial x}+u_{\mathrm{DY}} \cdot \frac{\partial T}{\partial y}\right]
$$

However, due to the non-linear solver used, the damped Newton iteration with the Armijo-Golstein line search, the formation of this workaround presented other challenges including a reduction to the damping factor in the line search beyond the allotted size of

$0.5^{16}$ therefore halting the program. This was resolved by refining the mesh and by using lumped Jacobian instead of the fixed Jacobian which is defaulted by PDETool at the cost of computational power and time.

\section{Varying Boundary Conditions}

Specifying boundary conditions using PDETool is done by entering a constant value or a known polynomial as a function of space into the coefficients in the Dirichlet and Neumann boundary conditions, Equation (3.42) and (3.43) respectively. However, in the case of the evaporative boundary condition, the pressure Neumann boundary condition is 
a function of thermal gradient. Since the final thermal gradient is unknown until solution convergence, it varies in space and at each iteration. To accommodate this variation at the evaporative boundary (updated value at each iteration), a function was created to generate boundary conditions requiring only boundary coordinates given by PDETool algorithm as an input. This function used the temperature field from the previous iteration (through the use of functions as described in Lack of Communication Section) as well as a surface interpolation renewing the boundary condition at every iteration step.

\section{Convection Boundary Condition}

To properly model an evaporative surface in a temperature field, a convection boundary condition should be applied since this will allow for an interfacial resistance [15].

$$
\lambda_{\mathrm{e}} \frac{\partial T}{\partial \hat{n}}=\alpha_{\text {evap }}\left(T-T_{\text {vap }}\right)
$$

where:

$\alpha_{\text {evap }}$ is thermal convection coefficient of evaporation

In phase change, the thermal convection coefficient is very high and has been assumed to be in the range of $3.0 \mathrm{E} 5 \mathrm{~W} / \mathrm{m}^{2}-\mathrm{K}$ by [11] and [15]. However, this boundary condition results in a nearly constant surface temperature with a near infinite heat transfer rate in a small region adjacent to the heating fin as demonstrated qualitatively in Figure 3.5. 


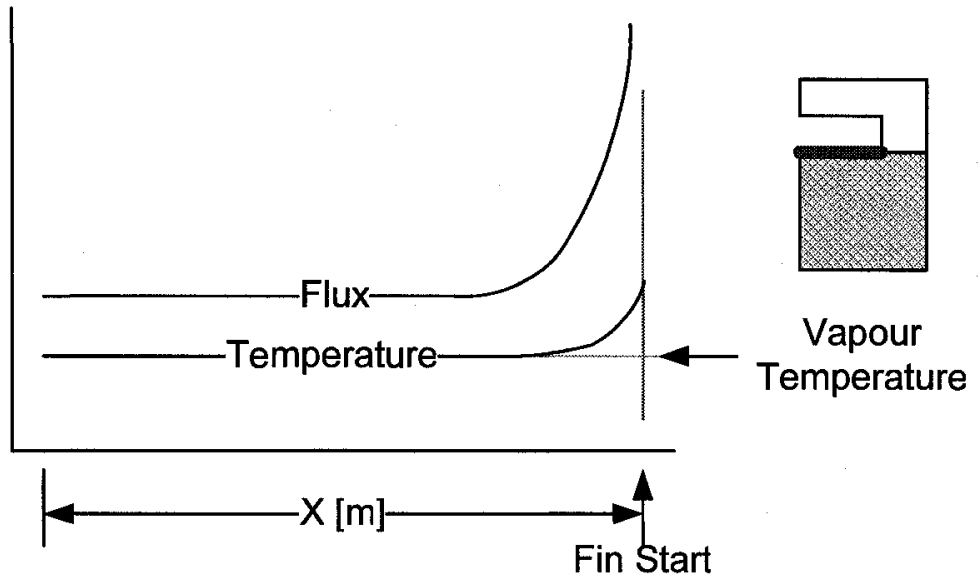

Figure 3.5 Temperature and flux trend on an evaporative surface using a convective boundary condition

Subsequently, the damped Newton iteration with the Armijo-Goldstein line search strategy sees this localized exponential growth in flux rate as an ill-conditioned boundary which causes very little change in the residuals between iterations therefore halting the program. If the mesh could be further reduced in the region of large gradients this challenge would be resolved though greatly increasing computational time; however, available computational strength was insufficient to apply this solution. To avoid this problem altogether, the vapour temperature was applied as the evaporative boundary condition. This boundary condition can be justified since there is very little change in vapour temperature at the evaporative surface. The disadvantage to this workaround is that it may cause localised numerical artefacts which results in underestimation of the evaporation intensity where the fin meets the wick due to possible abrupt change in temperature. 


\section{Post Processing}

When analysing the temperature and pressure field, a linear surfacing algorithm is used to sample values at specific $x-y$ coordinates since the solution only contains values at the mesh nodes. The griddata algorithm available in MATLAB was the tool of choice due to the rapidity and accuracy of the solution. It forces a surface as a function of $\mathrm{x}$ and $\mathrm{y}$ to the original mesh domain and solution using a Delaunay algorithm. However, the use of this surfacing tool can pose challenges when surfacing an area where the mesh is greatly skewed or when the location is subject to large gradients such as the contact point of the meniscus to the fin. At this location, the griddata algorithm will sample the solution to a value nearing that of the closest node. This effect can be seen when sampling values of thermal flux at the meniscus surface or fin-meniscus interface, Figure 3.6 and Figure 3.7 respectively. A solution to this challenge requires the sampling number to equal the node count in such regions as also demonstrated in Figure 3.6 and Figure 3.7. 


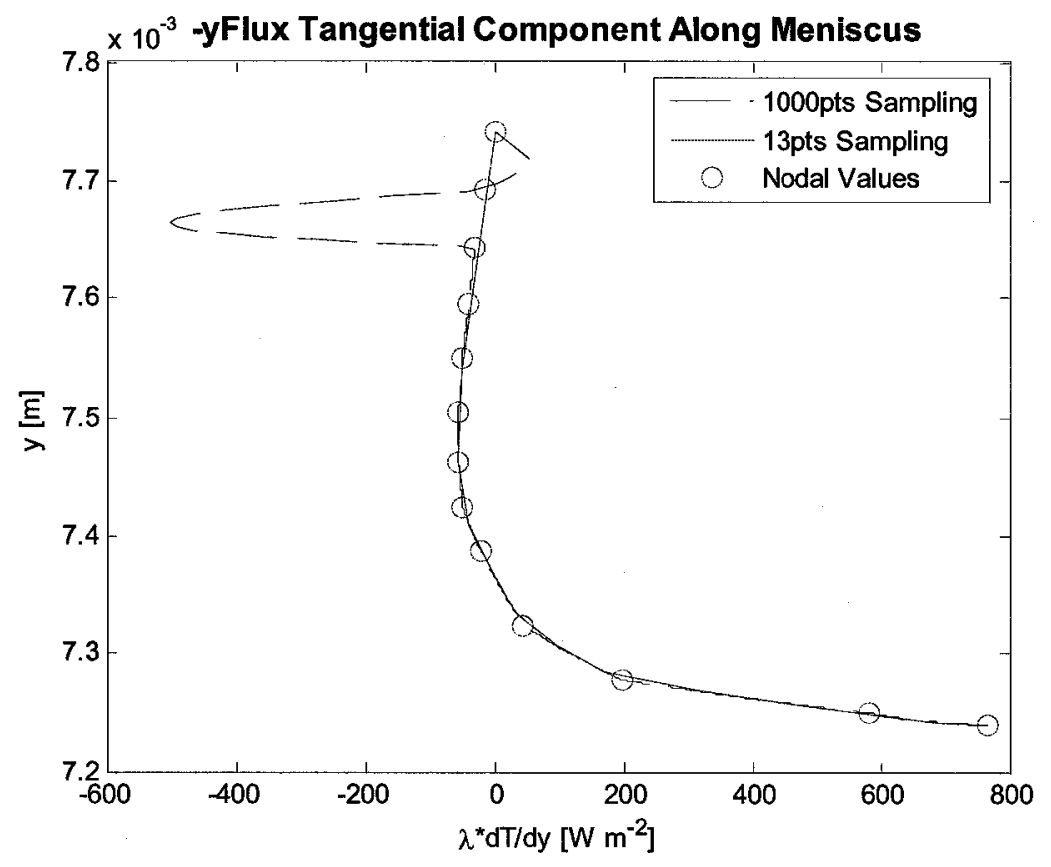

Figure 3.6 Y-component of thermal flux along meniscus surface

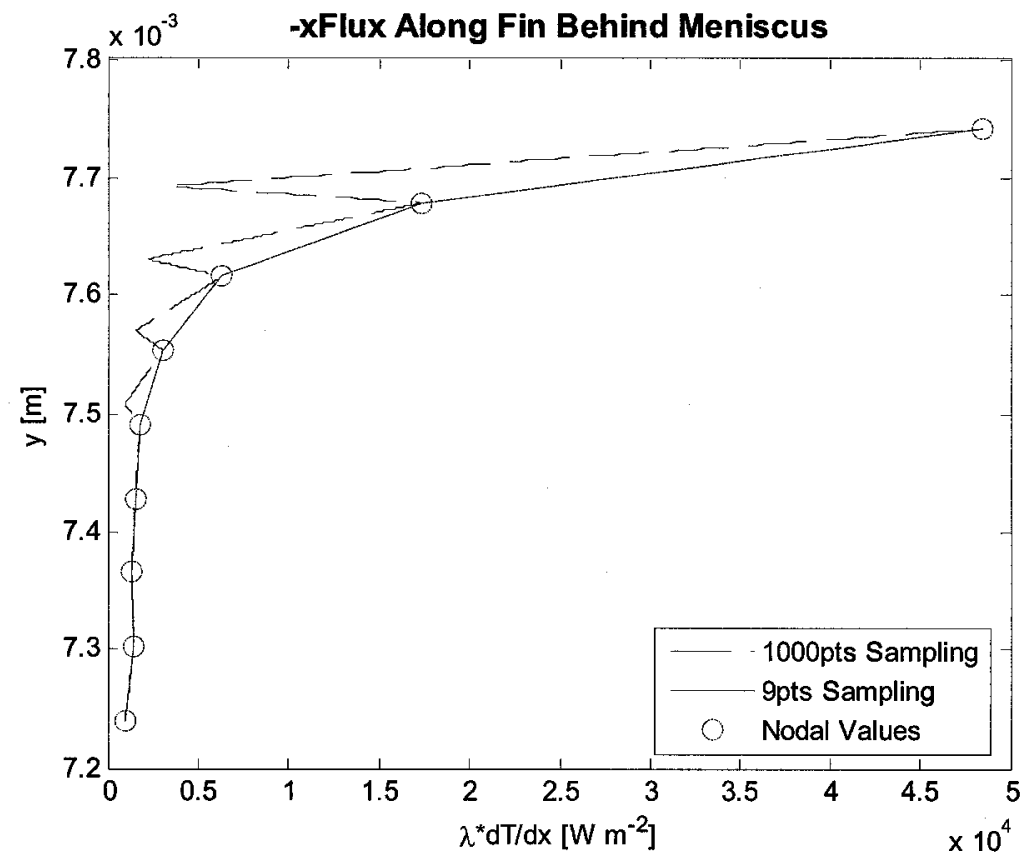

Figure 3.7 X-component of flux along meniscus-fin interface 


\subsubsection{Discretization of Domain}

A triangular mesh is generated on the computational domain using a Delaunay triangulation algorithm available as part of the PDETool. This algorithm computes the mesh by first creating a set of nodes along the boundary of the $2 \mathrm{D}$ domain to first triangulate the edges so that "sliver" triangles can be avoided or minimize. Nodes are then inserted into the centers of the edges of the bounded triangles, thus dividing every original triangle into four, see Figure 3.8. This division is repeated until the edge lengths are reduced below the maximum allowable length. Refining the mesh is also conducted in this fashion.

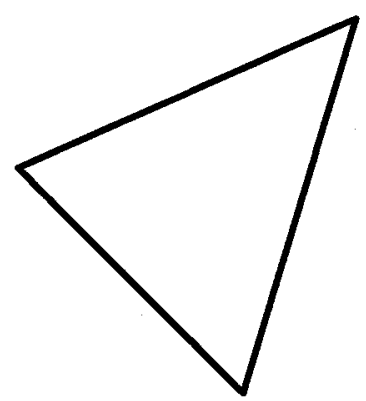

a)

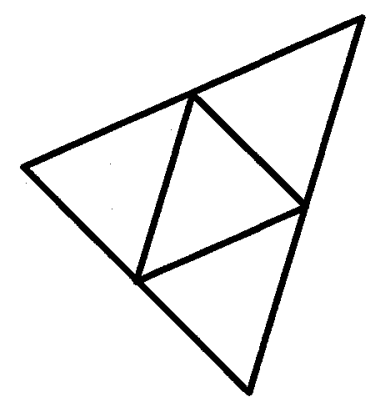

b)

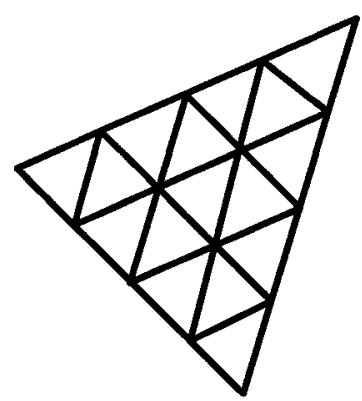

c)

Figure 3.8 Triangular mesh division: a) Original triangle, b) First division, c) Second division

\subsubsection{Solving Sequence}

The temperature and pressure field were solved separately; however, the solutions were coupled through an iterative sequence converging to less then $10^{-6}$ as identified in Section 3.6.2. The initial sequence followed for the first scenario (when the meniscus is non existent) follows the following steps: 
1. Create two meshes, one for the thermal computation and the other for the pressure computations;

2. Initialize the problem by solving the thermal diffusion equation in all domains;

3. Calculate the normal thermal gradient along the evaporation surface to derive the Neumann boundary condition for the Laplacian pressure equation;

4. Solve the Laplacian pressure equation;

5. Calculate the Darcian velocity;

6. Solve the diffusion equation in the casing and the convective-diffusion equation in the wick with the Darcian velocity;

7. Return to step 3 until all governing equations and boundary conditions are resolved to an RMS residual less then $10^{-6}$.

When this solution is achieved the second scenario (with the addition of the meniscus) can be resolved with the following sequence:

8. Calculate the pressure where the fin meets the wick to determine the geometry of the meniscus;

9. Re-mesh all domains (casing, wick and meniscus) for thermal computations;

10. Re-initialize the problem by solving the thermal diffusion equation in all domains;

11. Calculate the normal thermal gradient along the horizontal evaporation surface to derive the Neumann boundary condition for the Laplacian pressure equation;

12. Calculate the normal thermal gradient along the meniscus surface and with continuity derive the meniscus basal Neumann boundary condition for the Laplacian pressure equation; 
13. Solve the Laplacian pressure equation;

14. Calculate the Darcian velocity;

15. Solve the diffusion equation in the casing and the meniscus as well as the convective-diffusion equation in the wick with the Darcian velocity simultaneously;

16. Return to step 11 until all governing equations and boundary conditions are resolved to an RMS residual less then $10^{-6}$;

17. Return to step 8 until the radius of the meniscus is resolved to a difference less then $10^{-6}$.

The numerical solution sequence can be visualized in Figure 3.9 and Figure 3.10. 
Code Flow Chart (Without Meniscus)

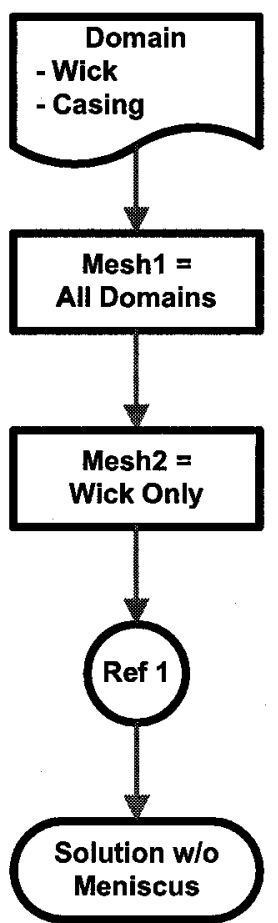

Code Flow Chart (With Meniscus)

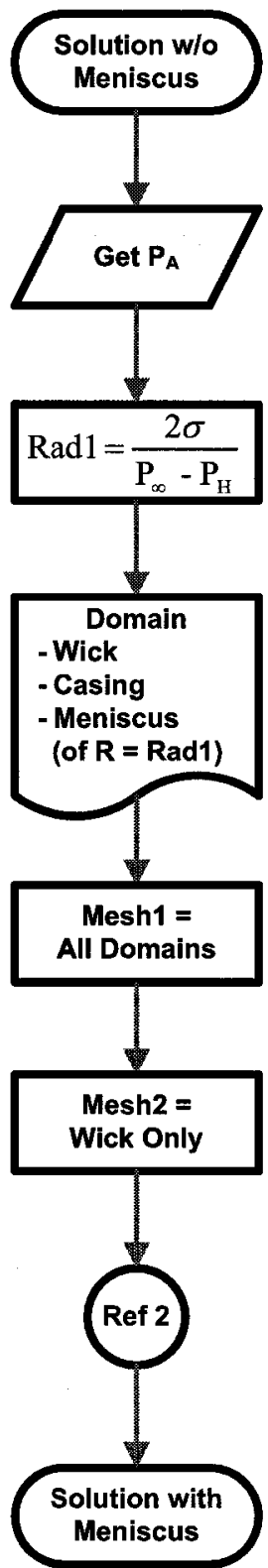

Figure 3.9 Numerical solution sequence flow charts (Part 1 of 2) 

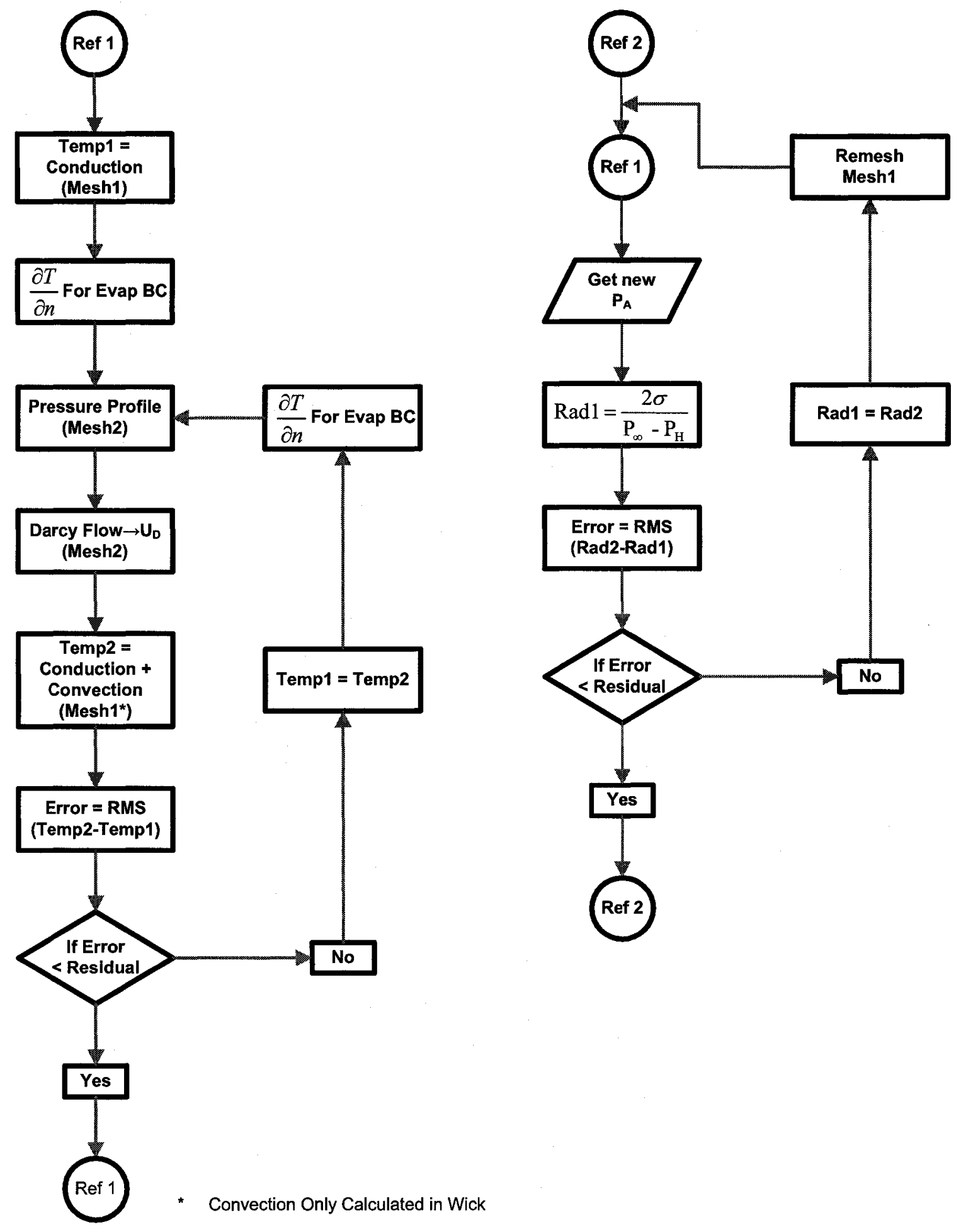

Figure 3.10 Numerical solution sequence flow charts (Part 2 of 2) 


\section{Chapter 4: Benchmark of Model}

As part of the validation of the modelling approach, it is always preferable to compare the model's results against experimental data. However, as stated previously Section 1.3, there is a lack of available experimental results in literature due to the difficulty in generating experimental data. Therefore, some aspects of the model are verified against the results of a commercial code, CFX.

\subsection{Porous Channel Flow with Partial Heated Surface}

This numerical problem is based on the numerical experimentation conducted by Jiang published in 2006 [9]. Jiang [9] numerically investigated the temperature profile within a porous channel comprised of sintered particles with a partially heated surface. Multiple particle and domain sizes were simulated with different applied heat loads and entrance velocities by means of conjugate heat transfer using a local non-equilibrium model of which the parameters and applied conditions are summarized in Table 4-1 and Table 4-2.

Table 4-1 Jiang's [9] geometrical parameters

\begin{tabular}{|c|c|c|}
\hline$[\mathrm{mm}]$ & Porosity & $\begin{array}{c}\text { Domain Size } \\
\text { [LXHXW] } \\
\text { [Dp] }\end{array}$ \\
\hline 0.6 & 0.402 & $10 \times 18 \times 1$ \\
\hline 1.2 & 0.444 & $5 \times 8 \times 1$ \\
\hline 1.7 & 0.463 & $5 \times 5 \times 2$ \\
\hline
\end{tabular}


Table 4-2 Jiang's [9] applied conditions

\begin{tabular}{ccccc}
\hline TRIAL & $\begin{array}{c}\mathbf{D p} \\
{[\mathrm{mm}]}\end{array}$ & $\begin{array}{c}\mathbf{T}_{\text {in }} \\
{[\mathrm{K}]}\end{array}$ & $\begin{array}{c}\mathbf{q}_{\text {in }} \\
{\left[\mathbf{W} / \mathbf{m}^{2}\right]}\end{array}$ & $\begin{array}{c}\mathbf{U}_{\text {in }} \\
{[\mathrm{mm} / \mathbf{s}]}\end{array}$ \\
\hline 1 & 1.7 & 300.00 & 31370 & 11.40 \\
2 & 1.7 & 300.00 & 132900 & 136.00 \\
3 & 1.2 & 289.17 & 14650 & 6.55 \\
4 & 1.2 & 289.74 & 138500 & 107.00 \\
5 & 0.6 & 290.66 & 19810 & 7.62 \\
6 & 0.6 & 289.93 & 105600 & 109.00 \\
\hline
\end{tabular}

Although the basic geometry and operating conditions from Jiang [9] are useful, the results could not be compared because of difference in modelling approach. The temperature range of Jiang's results compare well with the evaporator model's results but show discrepancies in the trends. The variation in trends can be explained by the fact that a laminar flow with an effective porous media and an equilibrium thermal model were used instead of Jiang's microscopic approach for modelling both the solid particles and fluid flow with a temperature slip condition at the solid-liquid interface of the porous structure. However, Jiang demonstrated that these discrepancies become negligible as the thermal conductivity of the solid particles and working fluid become similar in range. In contrast, the CFX solution was directly comparable with the modelling results as the same flow and boundary conditions were utilized.

For the numerical simulation in both the evaporator model and CFX, Jiang's geometry was lengthened as displayed in Figure 4.1 to ensure that the thermal convection was well developed and to prevent internal residual numerical artefact from the boundary condition. 


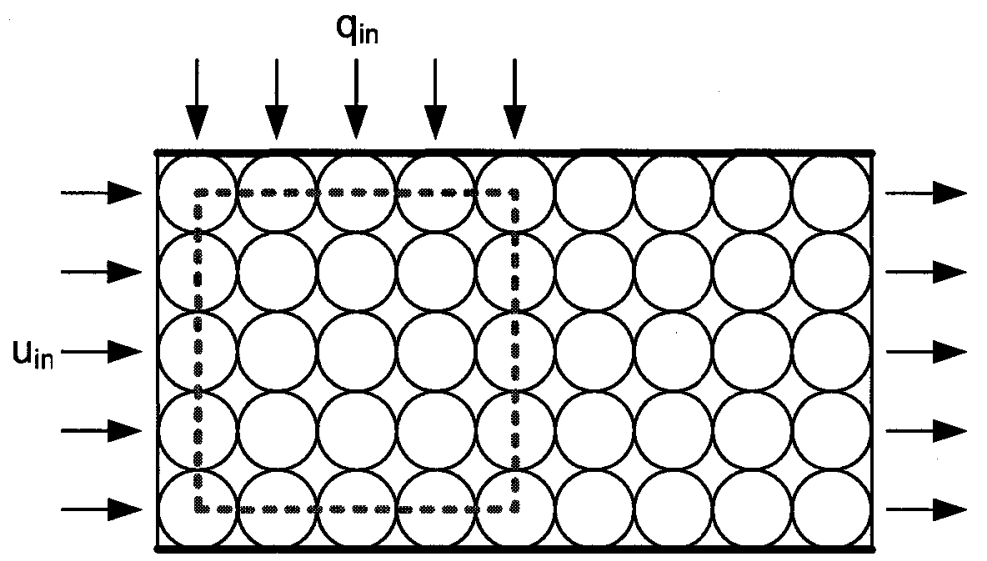

Figure 4.1 Schematic of lengthened porous channel domain: Dashed lines demonstrate Jiang's [9] numerical domain

Furthermore, with the particles and fluid being Tin-Bronze (CDA970) and water whose thermal conductivity values are $71 \mathrm{~W} / \mathrm{m}-\mathrm{K}$ [19] and $0.609 \mathrm{~W} / \mathrm{m}-\mathrm{K}$ [19] respectively, the values of effective thermal conductivity are calculated using Zehner and Schlunder's proposed correlation [8], Equation (2.9), as suggested by Jiang [9] and are summarized in Table 4-3.

Table 4-3 Effective thermal conductivity for Jiang's problem

\begin{tabular}{cc}
\hline Dp & $\begin{array}{c}\text { Effective Thermal } \\
\text { Conductivity, } \lambda_{\mathrm{e}} \\
\text { [W/m-K] }\end{array}$ \\
\hline \hline 0.6 & 5.624 \\
1.2 & 4.847 \\
1.7 & 4.533 \\
\hline
\end{tabular}

All trials in Table 4-2 were simulated. Trials 1,3 and 5 yielded very similar thermal fields (the solution of the third trial is presented in Figure 4.2). With the slower flow rates, conduction occurred which could be seen by the heat load penetrating into the 
porous domain. Convective dissipation is also seen in the longitudinal stretching of the applied heat load in space. When the flow rates were higher such as that of trials 2,4 and 6 (the solution of the fourth trial is presented in Figure 4.3), the convection overshadowed the diffusion and the heat load would barely penetrate the porous domain.
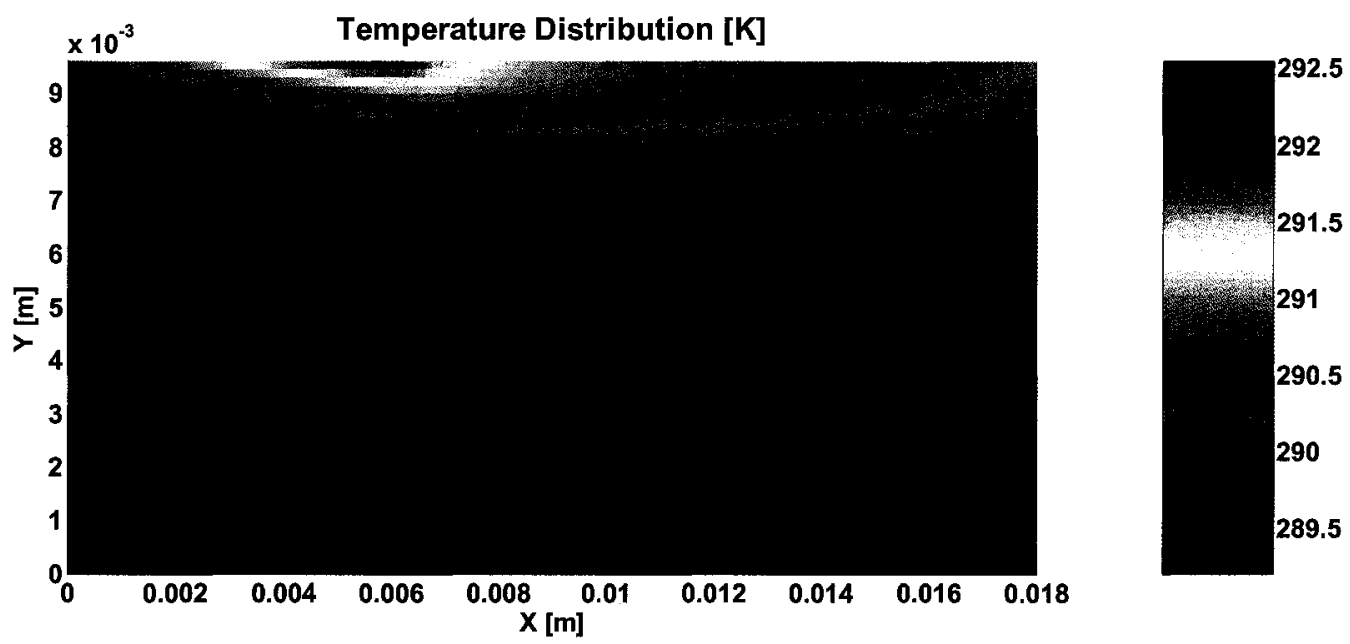

Figure 4.2 Temperature field of Jiang's trial 3 as simulated by the evaporator model
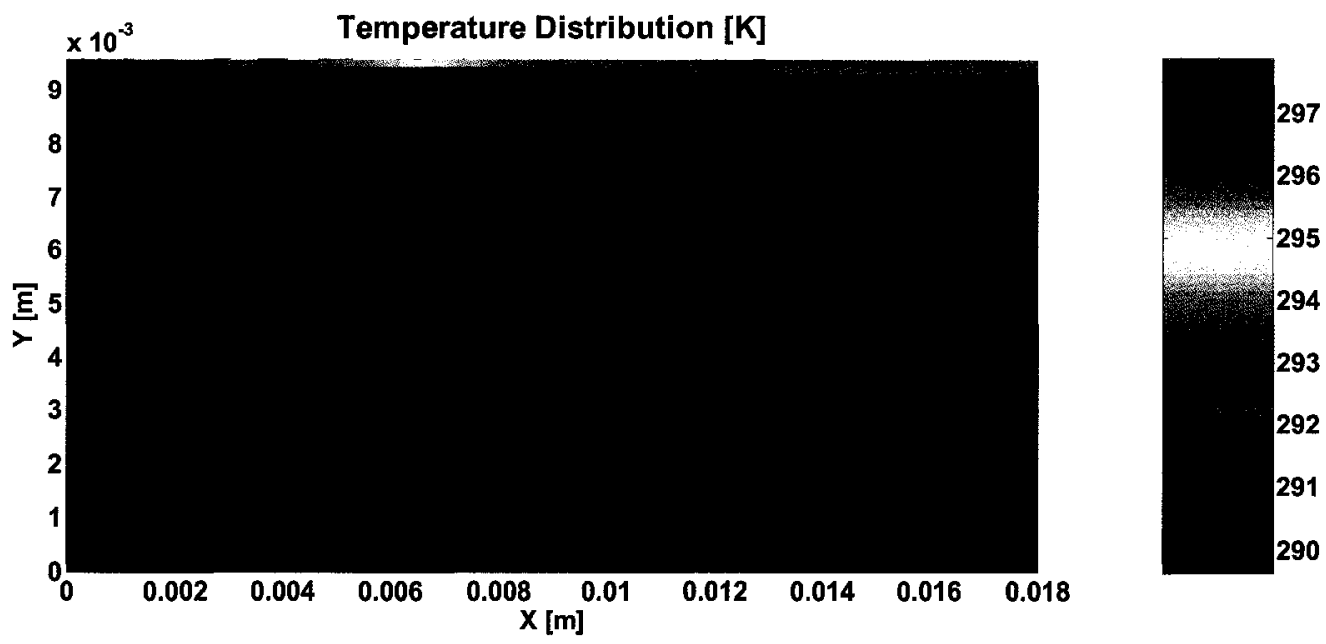

Figure 4.3 Temperature field of Jiang's trial 4 as simulated by the evaporator model

The temperature profile of the second row of particles obtained by CFX and the evaporator model are presented in Figure 4.4 demonstrating that the evaporator model 
produces results that are in very good agreement with that of CFX. This level of agreement was seen in all trials; therefore, it is assumed that the modelling process concerning the convective term has been well implemented and is valid.

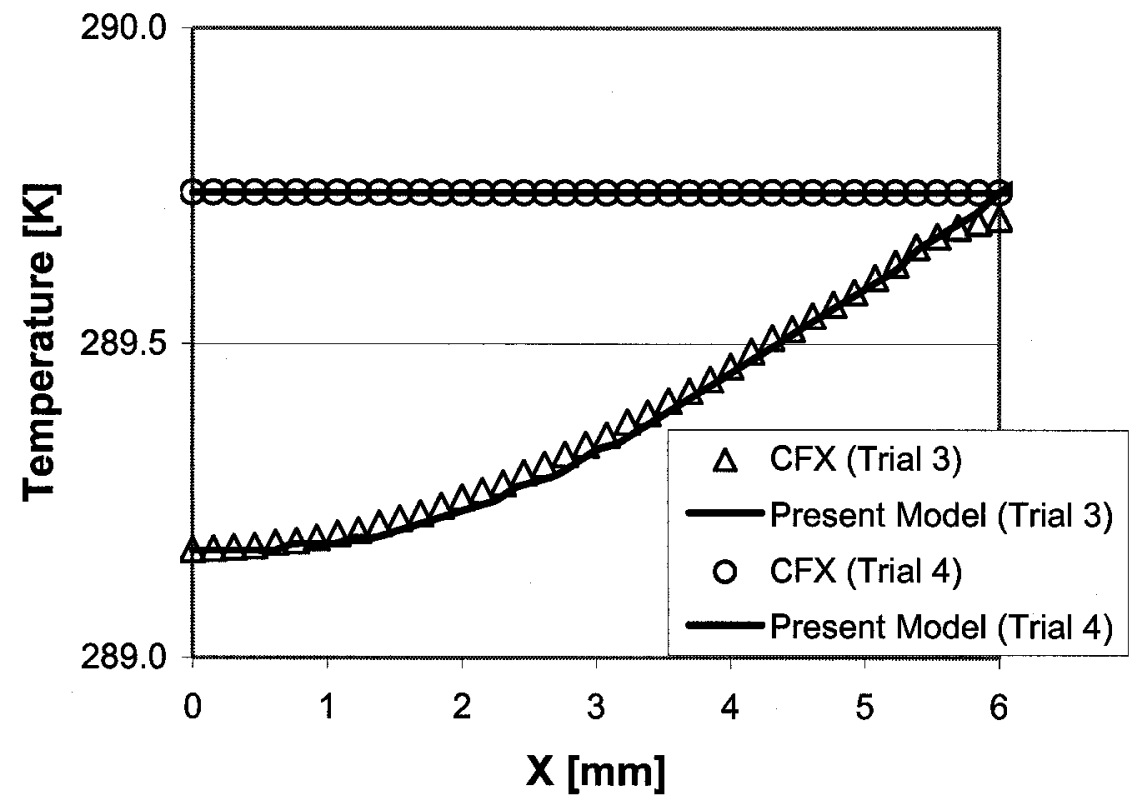

Figure 4.4 CFX and evaporator model temperature trend comparison (horizontal cut at second row of solid particles)

\subsection{Qualitative Verification of the Evaporative Surface}

Verification of the evaporation modelling process is essential since the loop heat pipe is exclusively driven by it. To verify if fluid flow driven by evaporation could be predicted, a test case was created. The test domain identified in Figure 4.5 consists of a tank with a heated feed at the bottom to keep the liquid level constant and an opening top surface for evaporation. The major assumptions applied are that boiling does not occur within the porous media and that it remains fully saturated at all times. The latter implies that the capillary forces compensate for the amount of liquid evaporating. 


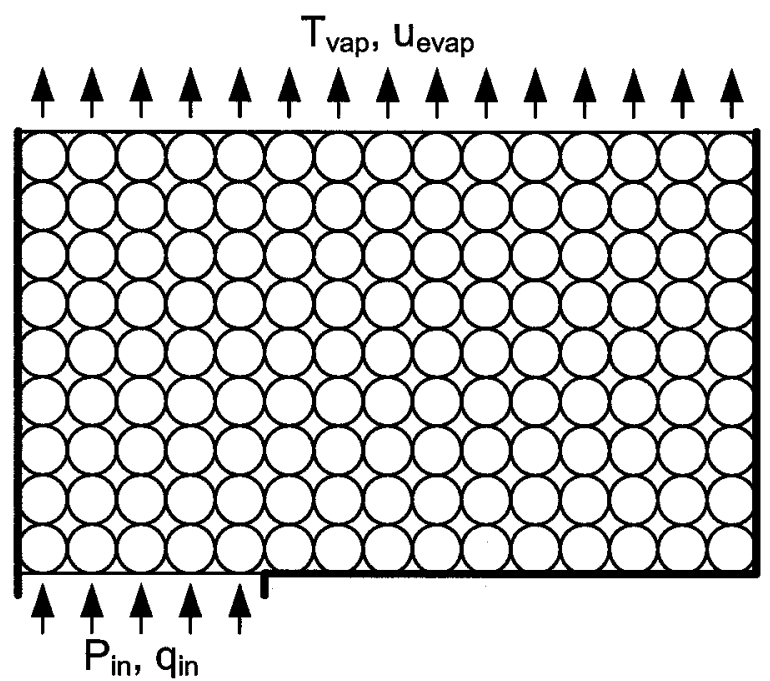

Figure 4.5 Schematic of evaporation test

Using the complete iterative process as described for the first scenario in Section 3.6.4, the same porous media parameters as trials 5 and 6 of Section 4.1 and the applied boundary condition as summarized in Table 4-4, a solution converged.

Table 4-4 Boundary condition for evaporation test

\begin{tabular}{ccccc}
\hline $\begin{array}{c}D p \\
{[\mathrm{~mm}]}\end{array}$ & $\varepsilon$ & $\begin{array}{c}\mathbf{P}_{\text {in }} \\
{[\mathrm{Pa}]}\end{array}$ & $\begin{array}{c}\mathbf{q}_{\text {in }} \\
{\left[\mathbf{W} / \mathbf{m}^{2}\right]}\end{array}$ & $\begin{array}{c}\mathbf{T}_{\text {vap }} \\
{[\mathrm{K}]}\end{array}$ \\
\hline 0.6 & 0.402 & 0 & 26630 & 377 \\
\hline
\end{tabular}

The temperature profile for the evaporation verification presented in Figure 4.6 demonstrates that the heat load diffused into the porous media from the open feed on the left towards the top surface. As such, the right side of the domain remains cooler. 


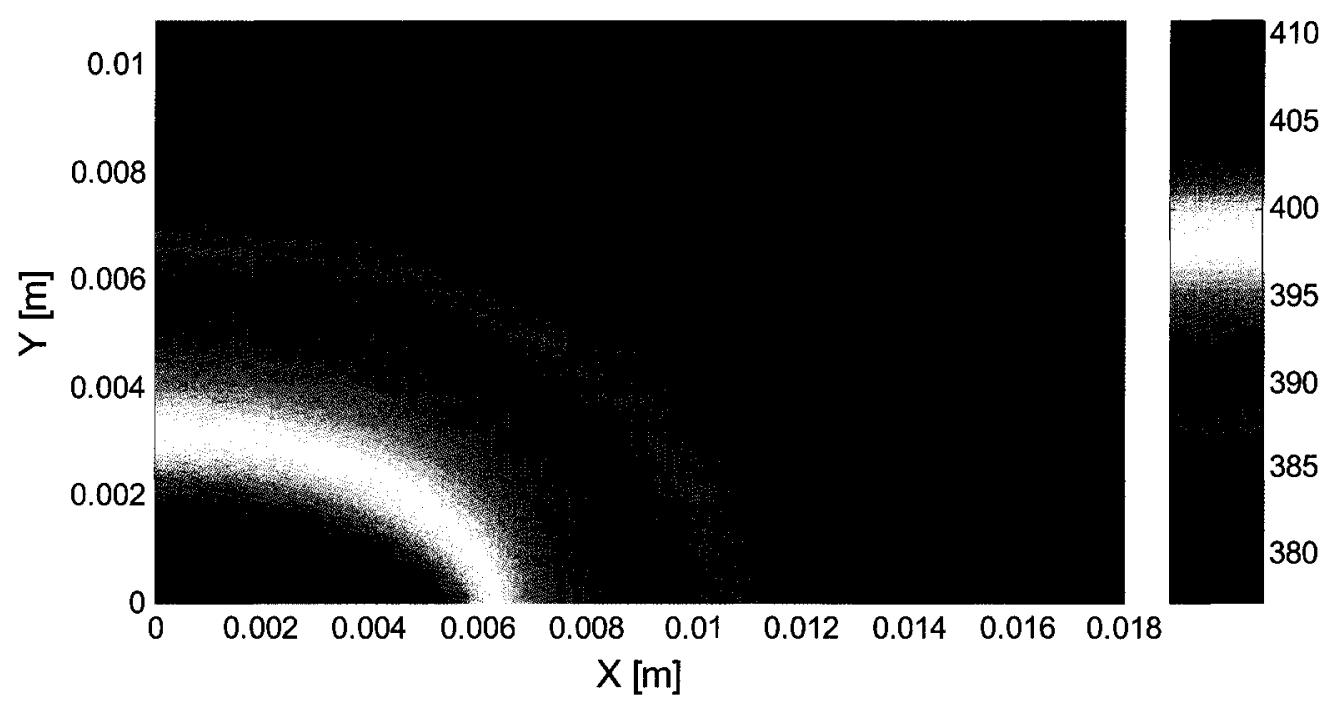

Figure 4.6 Temperature distribution [K] of evaporation test simulated by the evaporator model

The resulting flux distribution along the evaporative surface should give rise to an equivalent trend in liquid velocity due to their direct correlation as seen in Equation (3.20). As demonstrated in Figure 4.7, the evaporator model was indeed able to predict evaporation at the open surface with corresponding trends in agreement with the expected physical behaviour. Therefore it is assumed that the modelling process concerning evaporation has been well implemented. 


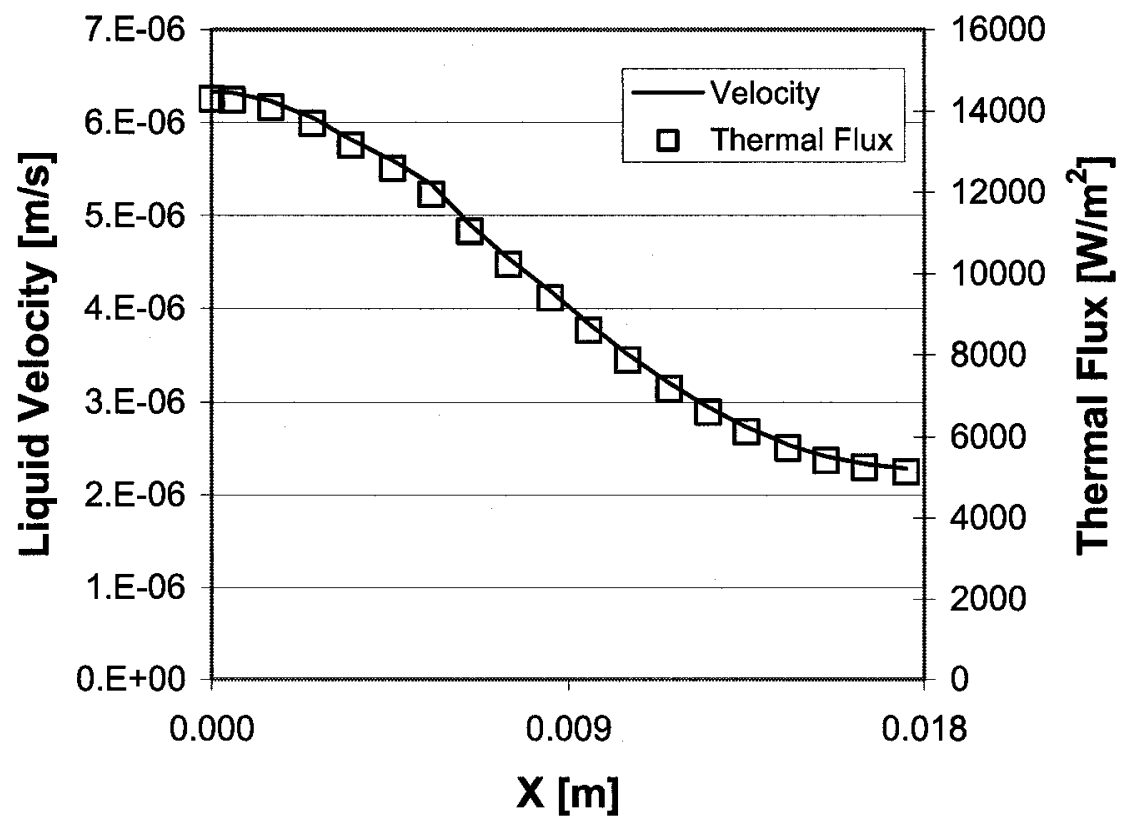

Figure 4.7 Liquid velocity and thermal flux at the evaporative surface of the evaporation test as simulated by the evaporator model 


\section{Chapter 5: Loop Heat Pipe Evaporator Model and Results}

\subsection{Preliminary Model Setup}

The evaporator model was written in a modular fashion. This simplified its use as it can be quickly modified to simulate different geometrical shapes or changes in physical parameters. However, for this investigation, a geometry and parameters had to be set. These are discussed in the following subsection.

\subsubsection{Model Dimensions}

For the flat type evaporator, three components being considered are the casing, the wick and the meniscus. These components are implemented as numerical domains by prescribing their shapes into the model. The casing is entered as a polygon with $(\mathrm{x}, \mathrm{y})$ coordinates at every corner. The wick is entered as a rectangle with points $\left(\mathrm{x}_{\min }, \mathrm{y}_{\min }\right)$ and $\left(\mathrm{x}_{\max }, \mathrm{y}_{\max }\right)$ specified. Care must be taken to ensure that the two components are such that they align perfectly to be considered two parts of one model. When the meniscus is added to the set of domains, a combination of a rectangle and circle is utilized. This is accomplished by adding a rectangle with all sides equal the radius length and subtracting a circle with the center coordinate $(\mathrm{x}, \mathrm{y})$ and radius equal to that of the meniscus from its top corner thus leaving the meniscus domain as demonstrated in Figure 5.1. 


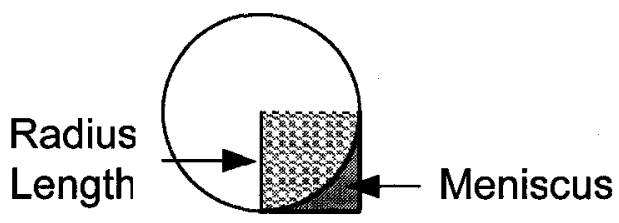

Figure 5.1 Creating a meniscus domain

The dimensions of the evaporator studied are summarized in Table 5-1.

Table 5-1 Dimensions of numerically investigated domain

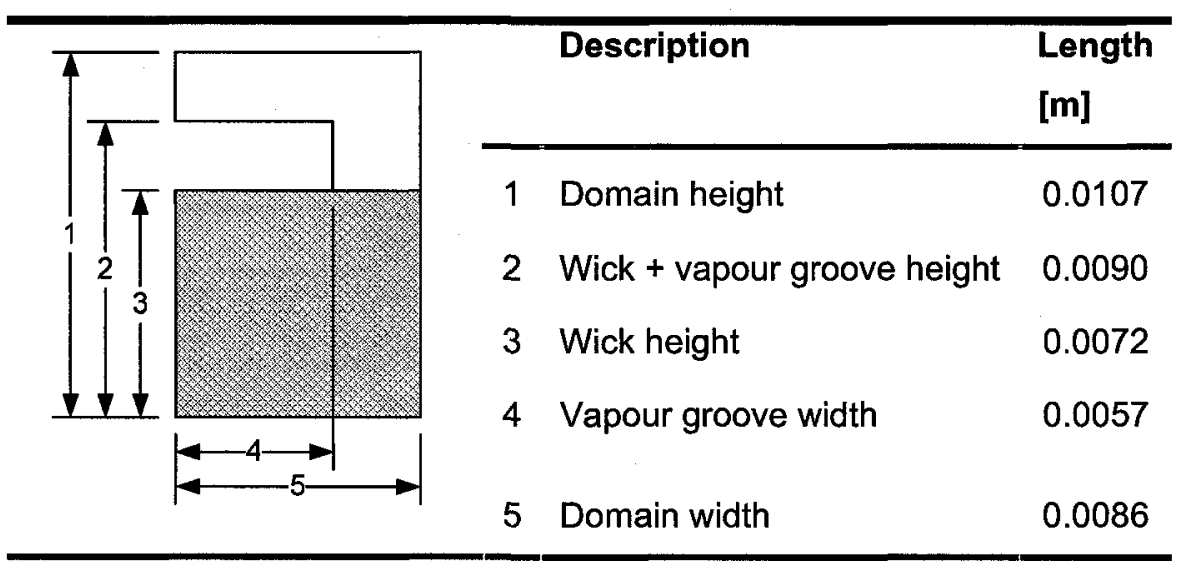

\subsubsection{Material Properties}

The chosen materials for this investigation were copper for the casing as well as the wick's solid matrix and water for the working fluid. Their constant parameters are entered directly into the forefront of main code as variables that can be called upon throughout the procedures. The parameters are summarized in Table 5-2. 
Table 5-2 Material properties

\begin{tabular}{lcc}
\hline Material Property & $\begin{array}{c}\text { Liquid } \\
\text { Water [19] }\end{array}$ & $\begin{array}{c}\text { Solid } \\
\text { Copper [19] }\end{array}$ \\
\hline Density, $\rho,\left[\mathrm{kg} / \mathrm{m}^{3}\right]$ & 998.23 & 8960 \\
Thermal Conductivity Coefficient, $\lambda,[\mathrm{W} / \mathrm{m}-\mathrm{K}]$ & 0.609 & 385 \\
Specific Heat Capacity, $C,[\mathrm{~J} / \mathrm{kg}-\mathrm{K}]$ & 4181.9 & 385 \\
Latent Heat of Vaporization, $h_{\mathrm{fg}},[\mathrm{J} / \mathrm{kg}]$ & 2258000 & $\mathrm{~N} / \mathrm{A}$ \\
Viscosity, $\mu,[\mathrm{kg} / \mathrm{m}-\mathrm{s}]$ & 0.001002 & $\mathrm{~N} / \mathrm{A}$ \\
Surface Tension, $\sigma,[\mathrm{N} / \mathrm{m}]$ & 0.0580 & N/A \\
\hline
\end{tabular}

\subsubsection{Wick Properties}

The wick properties were chosen to be the same as that of [14] as it facilitated the use of the one dimensional loop heat pipe model to derive the vapour parameters. As such, permeability and porosity are prescribed as $K=4 E-14 \mathrm{~m}^{2}$ and $\varepsilon=0.6$, respectively. Furthermore, the effective thermal conductivity was calculated to be $\lambda_{\mathrm{e}}=4.1619 \mathrm{~W} / \mathrm{m}-\mathrm{K}$ using the correlation proposed by Zehner and Schlunder [8], Equation (2.9).

\subsubsection{Fluid Parameters}

Using the one dimensional model reported by Kaya and Hoang [3], the vapour pressure as well as the liquid saturation temperature were determined as a function of applied heat flux which is demonstrated in Figure 5.2. The saturation temperature curve indicates that the investigated range of the applied heat flux is in the variable conductance mode of the loop heat pipe. In this mode the temperature decreases as the applied heat flux is increased due to the cold temperature of the returning fluid from the partially activated 
condenser [3]. At higher applied heat fluxes, when the condenser would be fully activated, the saturation temperature would start to increase.

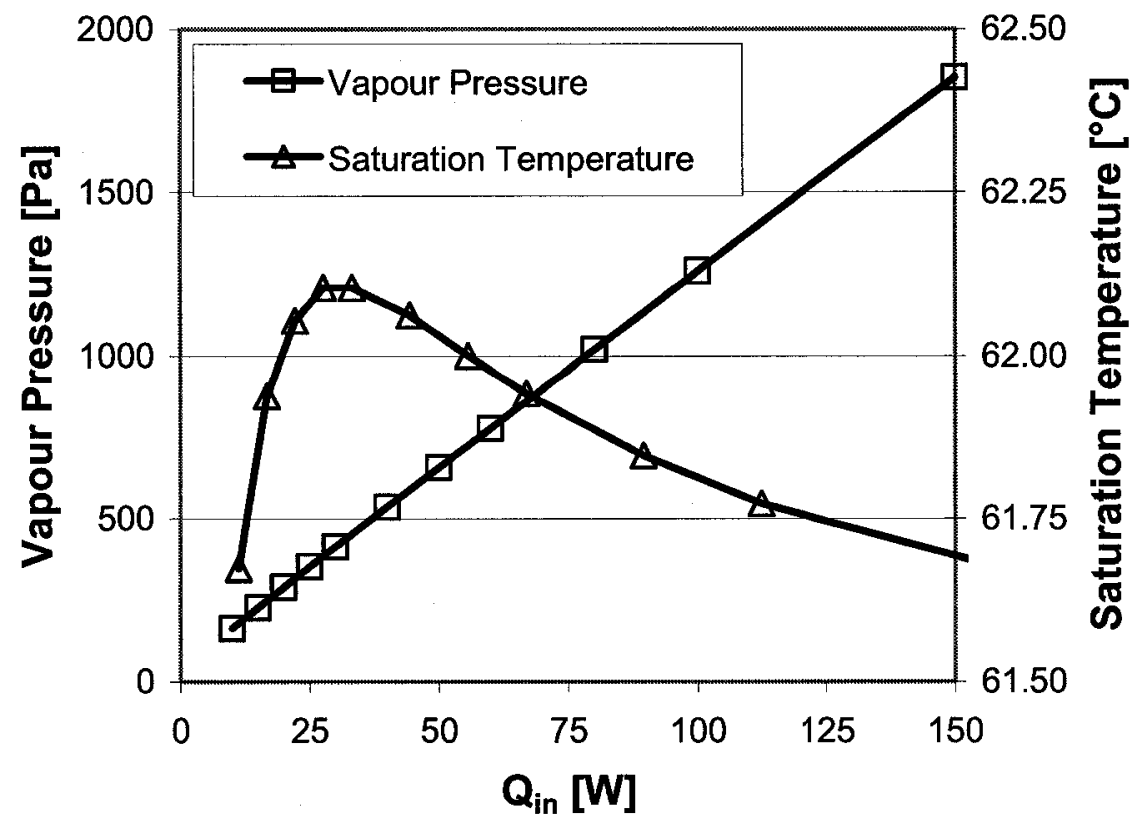

Figure 5.2 Prescribed vapour pressure and saturation temperature as determined by $1 D$ model reported in [3]

Since the liquid entering the evaporator is saturated, the saturation pressure was interpolated from water property tables using the saturation temperature, see Table 5-3. Table 5-3 Saturated water temperature and pressure (adapted from [20])

\begin{tabular}{cc}
\hline $\begin{array}{c}\text { Saturation Temperature } \\
{\left[{ }^{\circ} \mathrm{C}\right]}\end{array}$ & $\begin{array}{c}\text { Saturation Pressure } \\
{[\mathrm{kPa}]}\end{array}$ \\
\hline 40 & 7.384 \\
50 & 12.35 \\
60 & 19.94 \\
70 & 31.19 \\
80 & 47.39 \\
\hline
\end{tabular}


The vapour temperature at the evaporative surface will be superheated as it travels along the vapour groove. Since any amount of superheat can be realistically achieved with changes in evaporator length and evaluated cross sectional location, a superheat temperature profile ranging from $1.5^{\circ} \mathrm{C}$ to $2.5^{\circ} \mathrm{C}$ as demonstrated in Figure 5.3 was prescribed. This range is lower then that used in [15] of $3^{\circ} \mathrm{C}$ as a result of applied flux ranging from $15 \mathrm{~W}$ to $100 \mathrm{~W}$ as opposed to $100 \mathrm{~W}$ to $300 \mathrm{~W}$. Furthermore, this profile generated using a shooting method, generates similar results to that reported in literature.

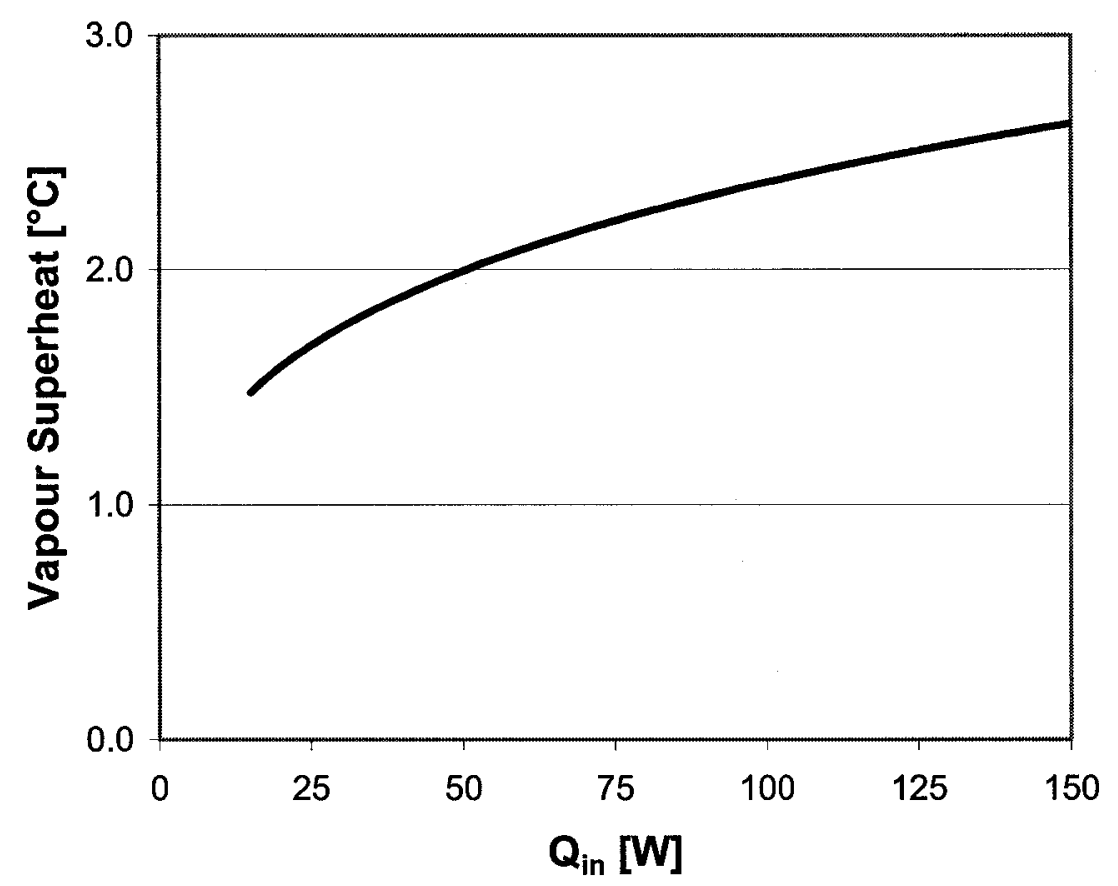

Figure 5.3 Prescribed superheat profile

\subsection{Grid Sensitivity}

To conduct the grid sensitivity analysis, a base mesh as well as three supplemental meshes having incremental levels of refinements were developed. Using the solution of the most refined mesh as the assumed true value, the percent difference of root-mean- 
square (RMS) values of the other coarser mesh solutions were calculated. This approach demonstrated that grid independence was reached with the crudest grid. Further investigation of localized changes shown in Figure 5.4, established that a nodal number nearing 2500 was necessary to achieve less than $3.0 \%$ local change with additional refinement when considering thermal flux as the examined parameter.
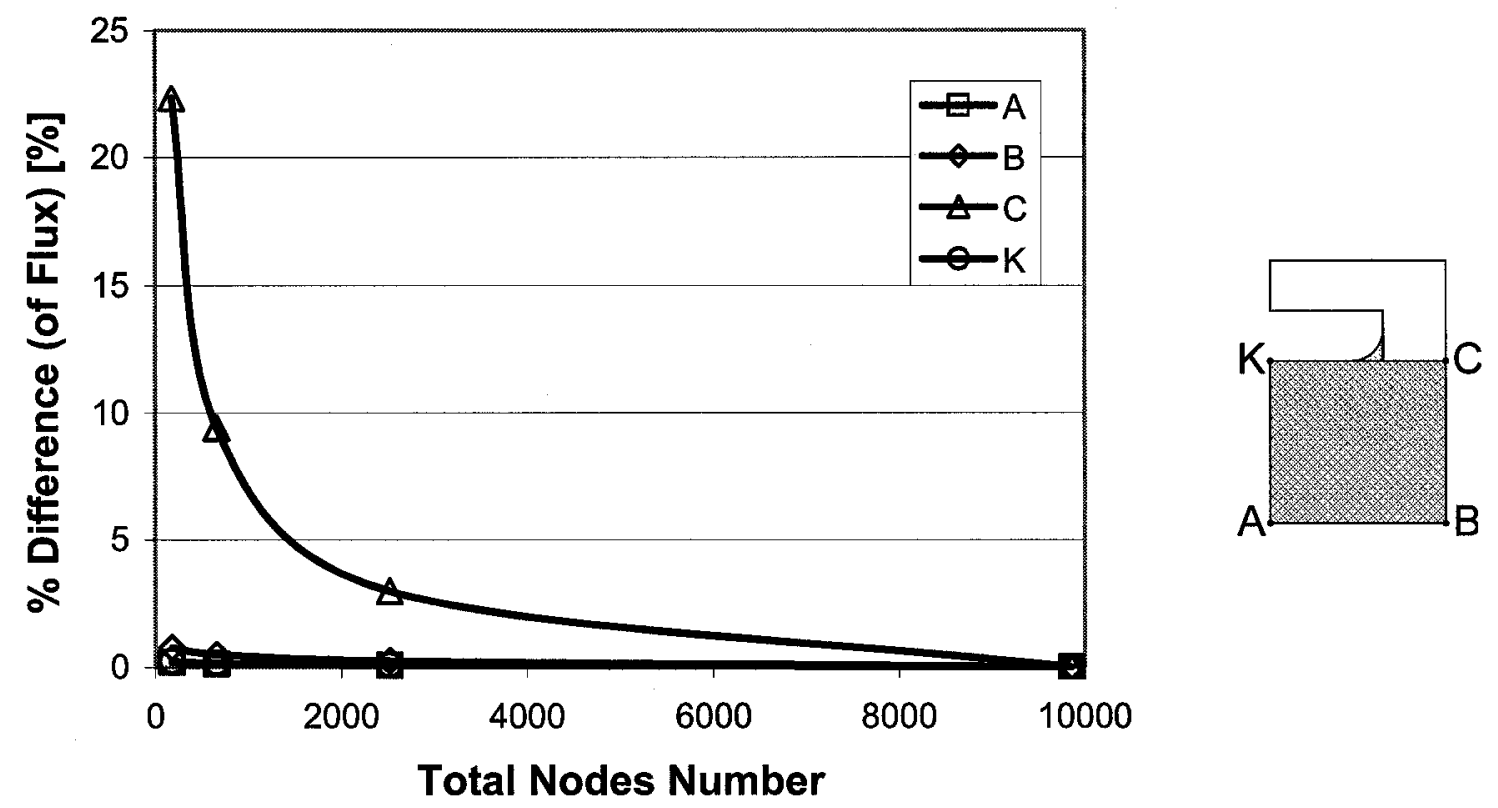

Figure 5.4 Grid independence study

As such, the second level of refinement which ranged from $\sim 2500$ to $\sim 4200$ nodes for applied heat fluxes of $15 \mathrm{~W}$ to $100 \mathrm{~W}$ respectively was deemed adequate for this investigation. To achieve any higher result of grid independence and solution accuracy would exceed the computational strength of the hardware used (Toshiba - Intel Centrino Duo-T2400@1.83GHz, 993 MHz, 1.00 GB RAM). The meshes utilized for modelling both scenarios with a $30 \mathrm{~W}$ applied heat flux are illustrated in Figure 5.5 and 5.6. 


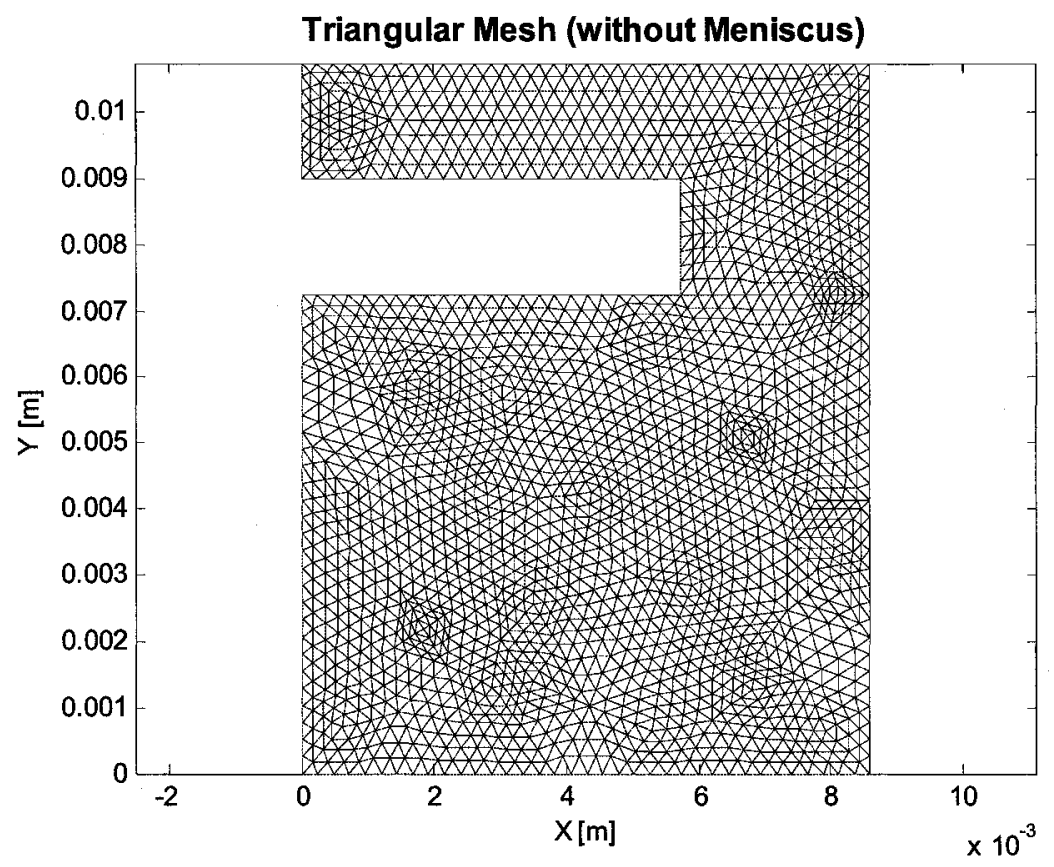

Figure 5.5 Mesh for $30 \mathrm{~W}$ applied heat flux without meniscus (2169 Nodes)

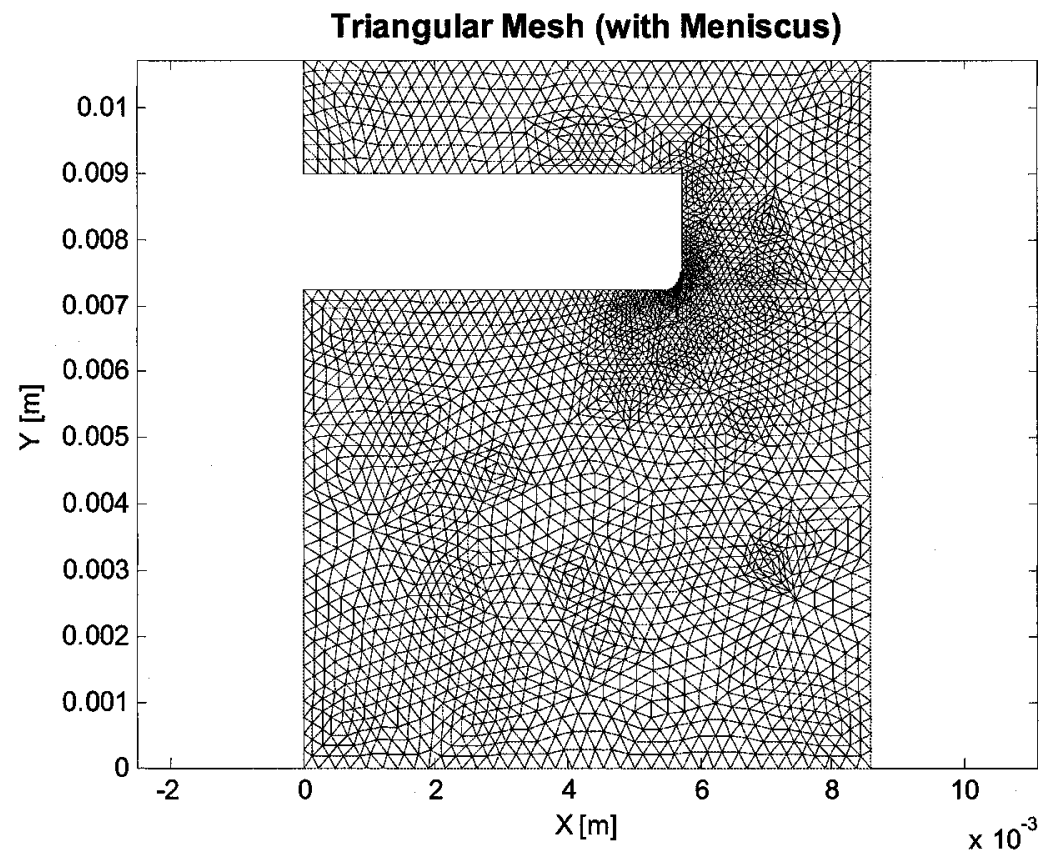

Figure 5.6 Mesh for $30 \mathrm{~W}$ applied heat flux with meniscus (2983 Nodes) 
Further localized refinements were necessary in the region of the meniscus to retain accuracy as the heat flux was increased.

\subsection{Scenario 1 (Evaporator with Saturated Wick)}

\subsubsection{Pressure Field}

The pressure field within the saturated wick is entirely determined by liquid evaporating at the liquid-vapour interface. As the evaporation rate increases, the pressure will drop accordingly to accept the changes in the internal fluid flow rates. However, once the pressure field within the wick is determined, a safety check against the Young-Laplace equation is conducted to ensure that the maximum capillary pressure has not been surpassed. It was observed that with low applied heat fluxes as investigated in this thesis, the maximum capillary pressure was never surpassed.

The pressure drop across the wick for all tested values of applied heat flux gave similar contour plots to that of Figure 5.7 where the lowest drop occurs as expected at the evaporation surface but with different magnitudes which is congruent to the trends of [15] with that of the fully saturated wick. These trends differ from that of [16] which assumes constant thin film evaporation under the heating fin therefore being the location of lowest pressure drop. 


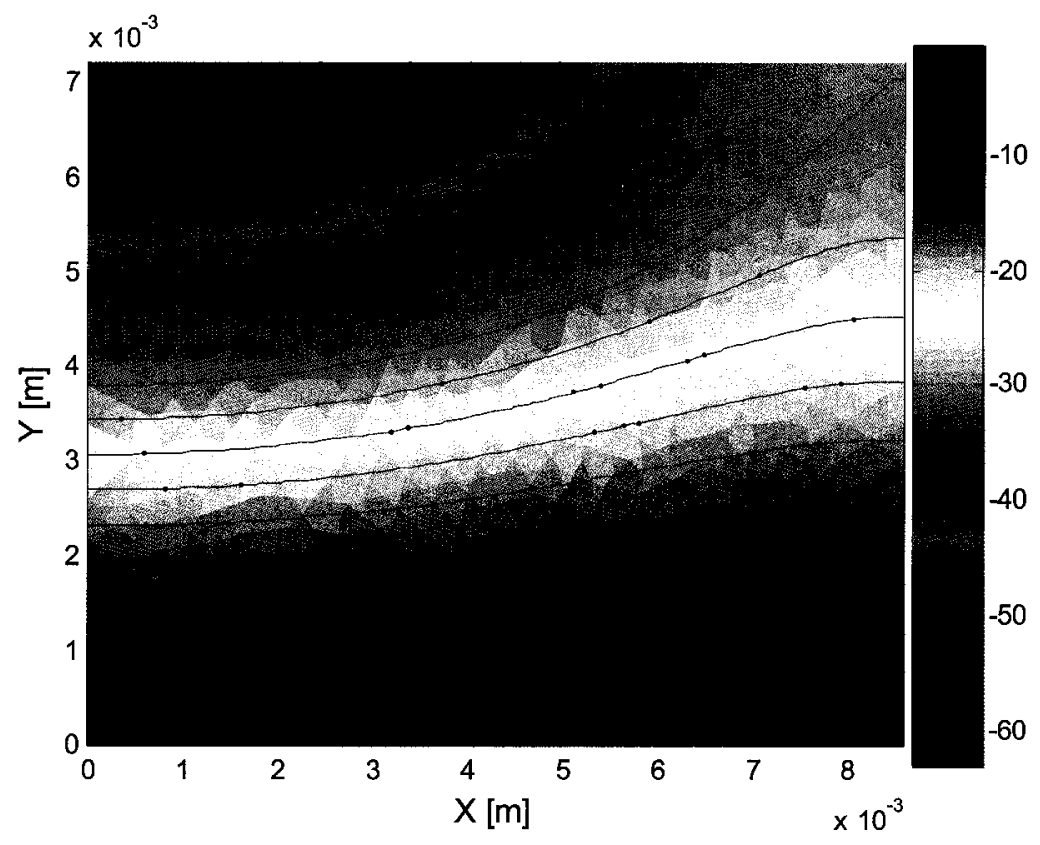

Figure 5.7 Pressure drop [Pa] in the wick for the saturated wick (30 $\mathrm{W}$ applied heat flux heating fin lies on $x=0.0057 \mathrm{~m}-0.0086 \mathrm{~m}$ at $\mathrm{y}=0.0072 \mathrm{~m}$ )

From the pressure field, the fluid flow can be directly established keeping in mind that the streamlines are perpendicular to isobars and it is the distance between the isobars that dictate the magnitude of velocity as described by Darcy's Law, Equation(2.2). Therefore from Figure 5.7, it can be seen that fluid enters the wick at a nearly constant velocity. As the fluid penetrates deeper into the wick, the fluid flow starts to increasingly curve towards the liquid-vapour interface where evaporation occurs.

The source of heat which induces evaporation at this location is twofold: heat transferred from the heating fin into the wick therefore heating the interface from below and causing higher evaporation intensity nearest the fin and heat transferred from the superheated vapour to the interface from above. At low heat flux with the present 
assumptions and boundary conditions, the results demonstrate that the evaporative intensity is largest nearest the center of the vapour groove indicating evaporation caused by the vapour supersedes that caused by the heating fin. This effect is reversed when simulating increasingly larger applied fluxes where at $100 \mathrm{~W}$ a balance is nearly achieved. However, this trend is directly related to the prescribed vapour temperature as a boundary condition. As the vapour temperature is reduced in the model, evaporation caused by the fin becomes more significant though little change is experienced by the overall pressure field. Therefore, the uncertainty in the exact vapour superheat temperature at one specific cross sectional location renders it impossible to discern whether this trend is an artefact of the boundary condition or is an actuality of the physical problem.

Velocity component results directly fed into the thermal convection-diffusion submodel are displayed in Figure 5.8.

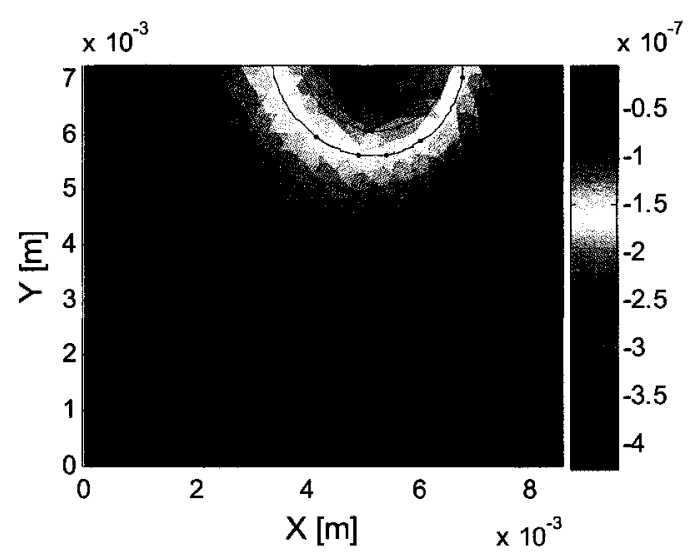

a)

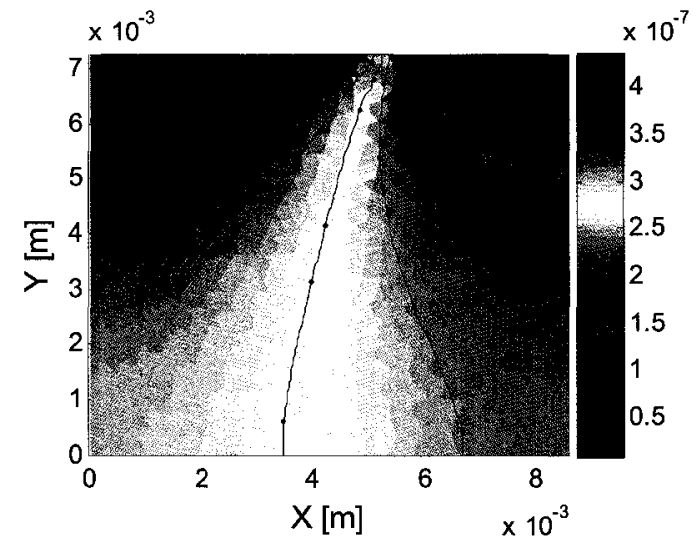

b)

Figure 5.8 Velocity field as determined from pressure drop (30 $\mathrm{W}$ applied heat flux heating fin lies on $x=0.0057 \mathrm{~m}-\mathbf{0 . 0 0 8 6} \mathrm{m}$ at $\mathrm{y}=0.0072 \mathrm{~m}$ ): a) $\mathrm{x}$-component, b) $\mathrm{y}$ component 
These contour plots indicate an average magnitude of velocity of $2.8 \mathrm{E}-7 \mathrm{~m} / \mathrm{s}$ for a $30 \mathrm{~W}$ applied heat flux which seems to be in the appropriate range when compared with [12] and [15] and taking in consideration the different working fluid and lower applied flux. Once again, due to the built-in assumptions of [16], the results displayed here differ by a couple orders of magnitude.

\subsubsection{Temperature Field}

The temperature field is much more complex to understand as it is subject to multiple effects. Evaporation at the evaporating surface causes the release of latent heat of vaporization therefore acting as a thermal sink. Furthermore, evaporation causes a fluid flow within the porous media therefore convecting the heat from both the heating fin as well as the superheated vapour towards the liquid-vapour interface increasing the effectiveness of the evaporator and also forcing the fluid entering the porous media to retain its cooler temperature further into the wick. Though the range of applied heat flux investigated in this thesis did not create sufficient fluid flow to observe convection effects (there was an RMS difference of $0.00038^{\circ} \mathrm{C}$ to $0.0055^{\circ} \mathrm{C}$ between numerical solution of temperature fields resulting from pure conduction to that of convective analysis for the first scenario and $0.027^{\circ} \mathrm{C}$ to $0.278{ }^{\circ} \mathrm{C}$ RMS difference for the second scenario for a range of applied heat flux of $15 \mathrm{~W}$ to $100 \mathrm{~W}),[16]$ suggested that as the applied heat flux increased, the heated region of the wick would be intensified near the evaporative surface. Finally, the thermal conductivity of the wick dictates the penetration depth of the thermal flux. As the thermal conductivity increases, the thermal flux of the heating fin and the superheated vapour will penetrate deeper into the wick therefore lowering the 
overall temperature of the evaporator as well as lowering the evaporation rate at the evaporative surface.

The temperature field for all tested values of applied heat flux gave similar contour plots to that of Figure 5.9 with different magnitudes. The wick temperature gradually increases almost linearly from saturation temperature of the incoming fluid to the vapour temperature at the evaporative surface due to low convection except in the region directly under the heating fin where the two-dimensional thermal penetration and flux curvature effects summarized in Section 2.2.1 are seen. These described trends strongly agree with those of $[11][12][15]$ and [16]. The only deviation from the latter three reported trends comes from the horizontal isotherms at the evaporative surface due to the constant vapour temperature boundary condition.

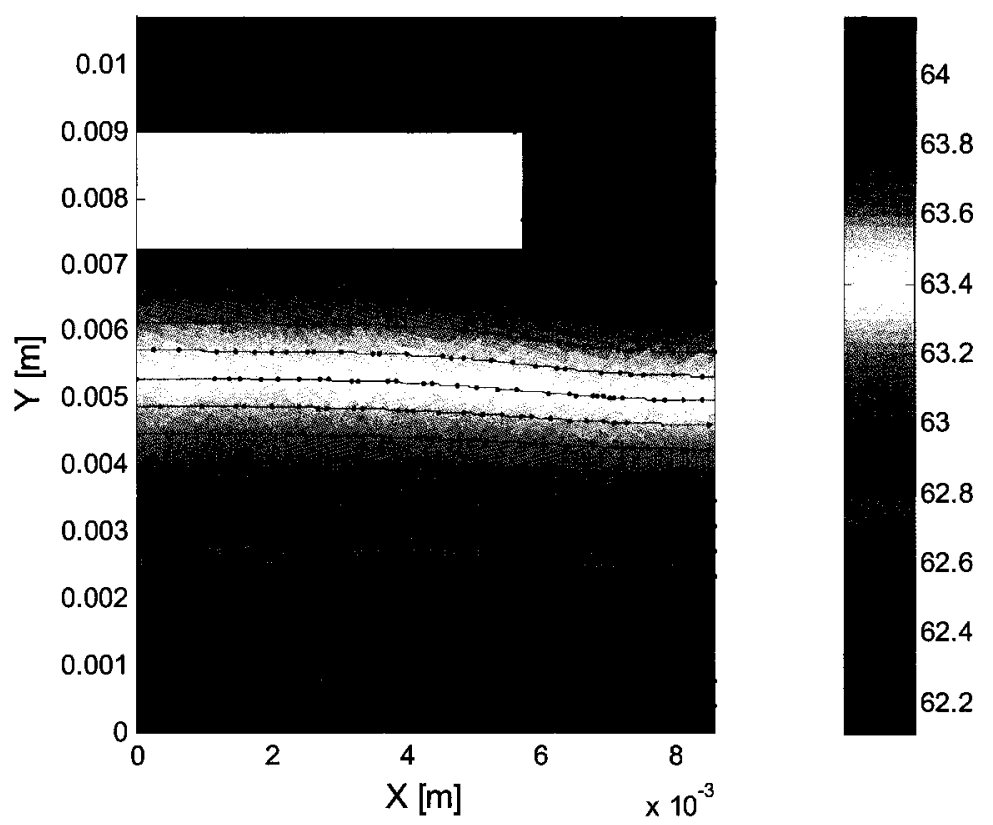

Figure 5.9 Temperature field $\left[{ }^{\circ} \mathrm{C}\right]$ for the saturated wick case ( $30 \mathrm{~W}$ applied heat flux) 
The temperature profile of the casing demonstrates that an increase in applied flux will increase the overall temperature of the casing. However, the region over the vapour groove will experience a higher temperature, as demonstrated in Figure 5.10, as the vapour groove offers a high thermal resistance comparatively to the fin. These trends are in agreement with [15].

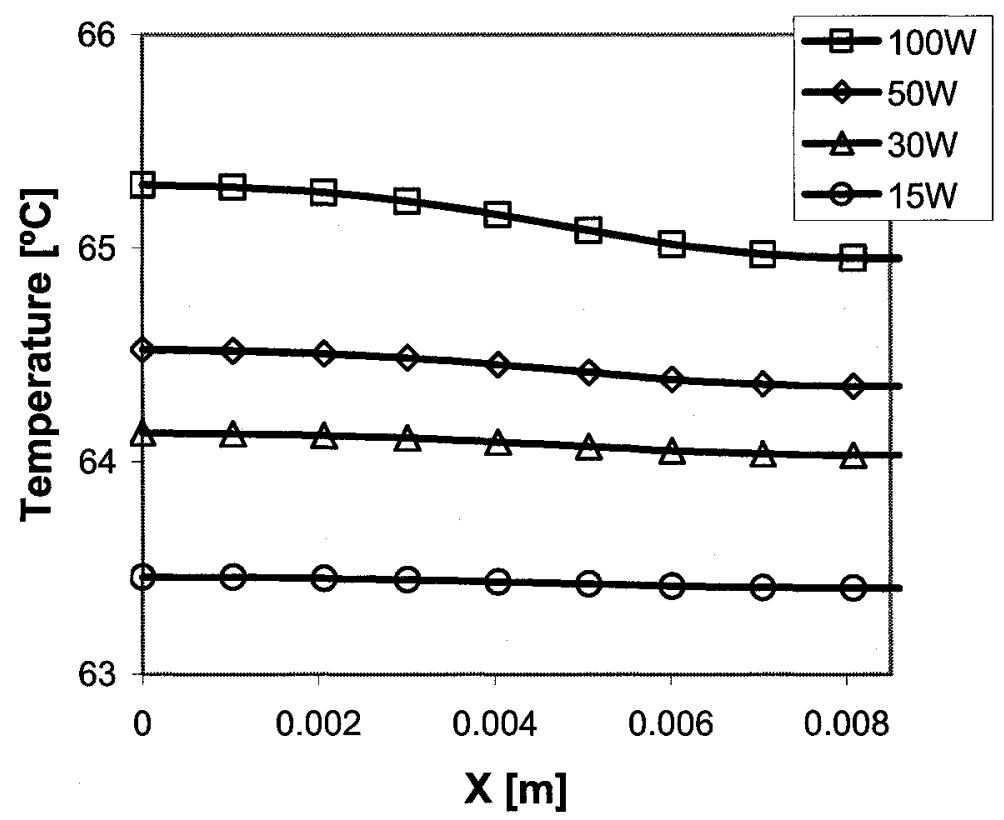

Figure 5.10 Casing temperature profile

\subsection{Scenario 2 (Evaporator with Meniscus)}

\subsubsection{Pressure Field}

The pressure field within the saturated wick with the presence of the meniscus is very different than that without meniscus. It can be seen in Figure 5.11, that the basal location of the meniscus exhibits the largest pressure drop, therefore, it is also the area of largest evaporation. As the applied heat flux is increased the meniscus diminishes in size 
therefore focusing and intensifying this evaporation effect which is similar to the findings of [11].

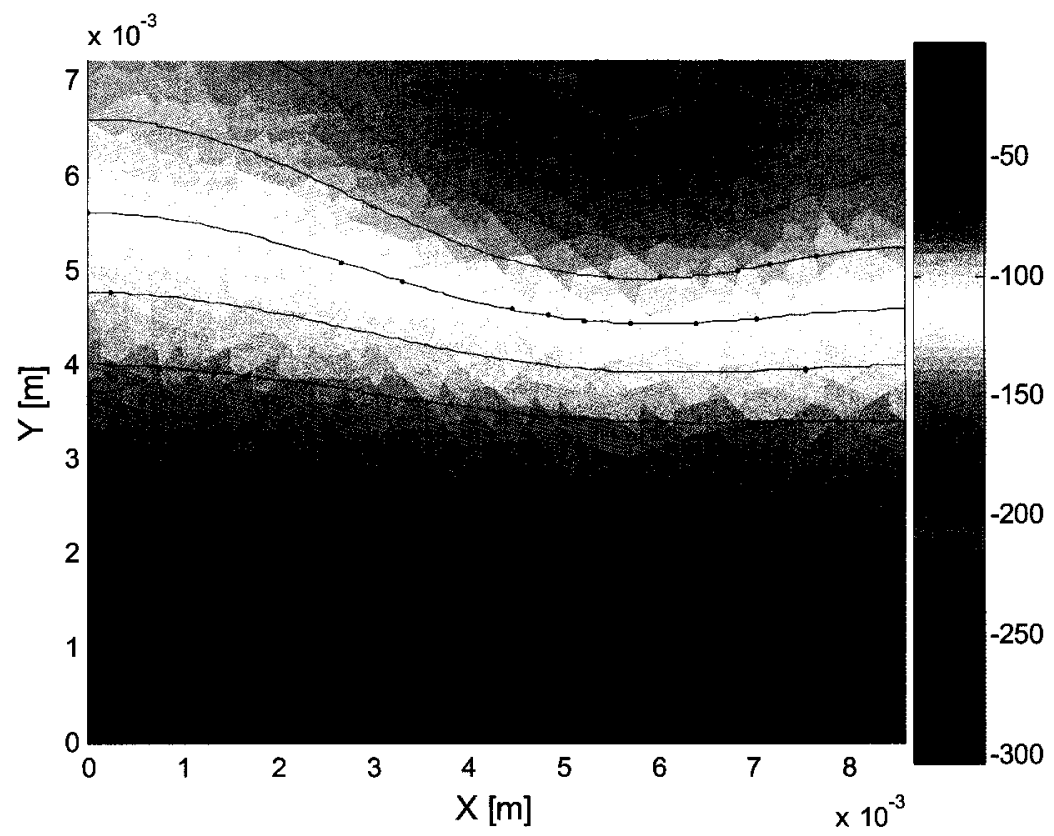

Figure 5.11 Pressure field [Pa] with presence of meniscus (30 W applied heat flux - heating fin lies on $\mathrm{x}=0.0057 \mathrm{~m}-0.0086 \mathrm{~m}$ at $\mathrm{y}=0.0072 \mathrm{~m}$ )

From this pressure field, the isobars indicate that fluid enters the wick at a nearly constant velocity similarly to that of Section 5.3.1. However, as the fluid penetrates deeper into the wick, the fluid flow starts to veer towards the basal location of the meniscus which is similar to the findings of [11]. Figure 5.12, demonstrates the velocity components of which a definite overall velocity increase can be seen. Furthermore, a direct comparison of the liquid velocity at the evaporative surface between results including and excluding the meniscus is shown in Figure 5.13 which establishes that the only change in evaporation rate occurs at the localized region of the meniscus. 


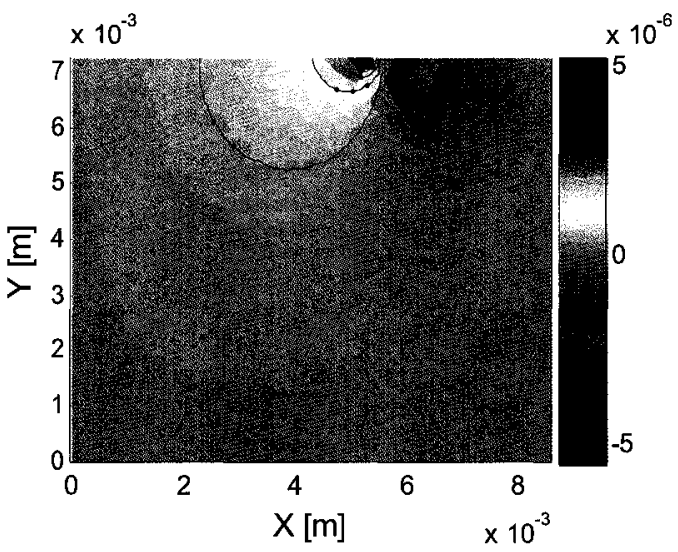

a)

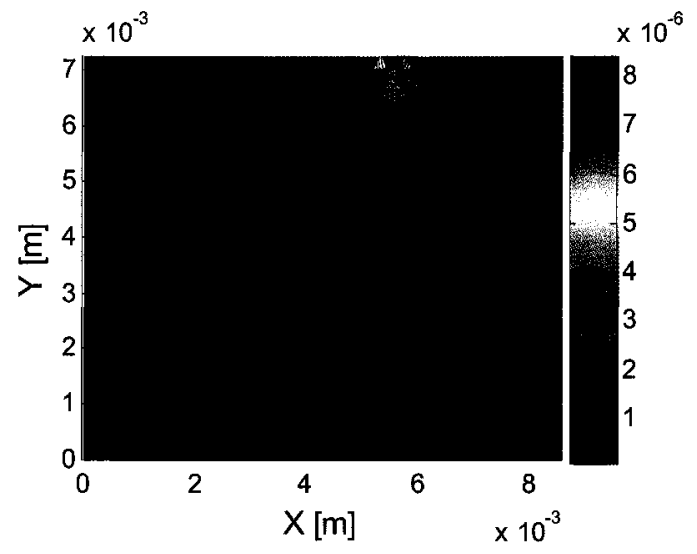

b)

Figure 5.12 Velocity field with presence of meniscus ( $30 \mathrm{~W}$ applied heat flux - heating fin lies on $x=0.0057 \mathrm{~m}-0.0086 \mathrm{~m}$ at $\mathrm{y}=0.0072 \mathrm{~m}$ ): a) $\mathrm{x}$-component, b) y-component

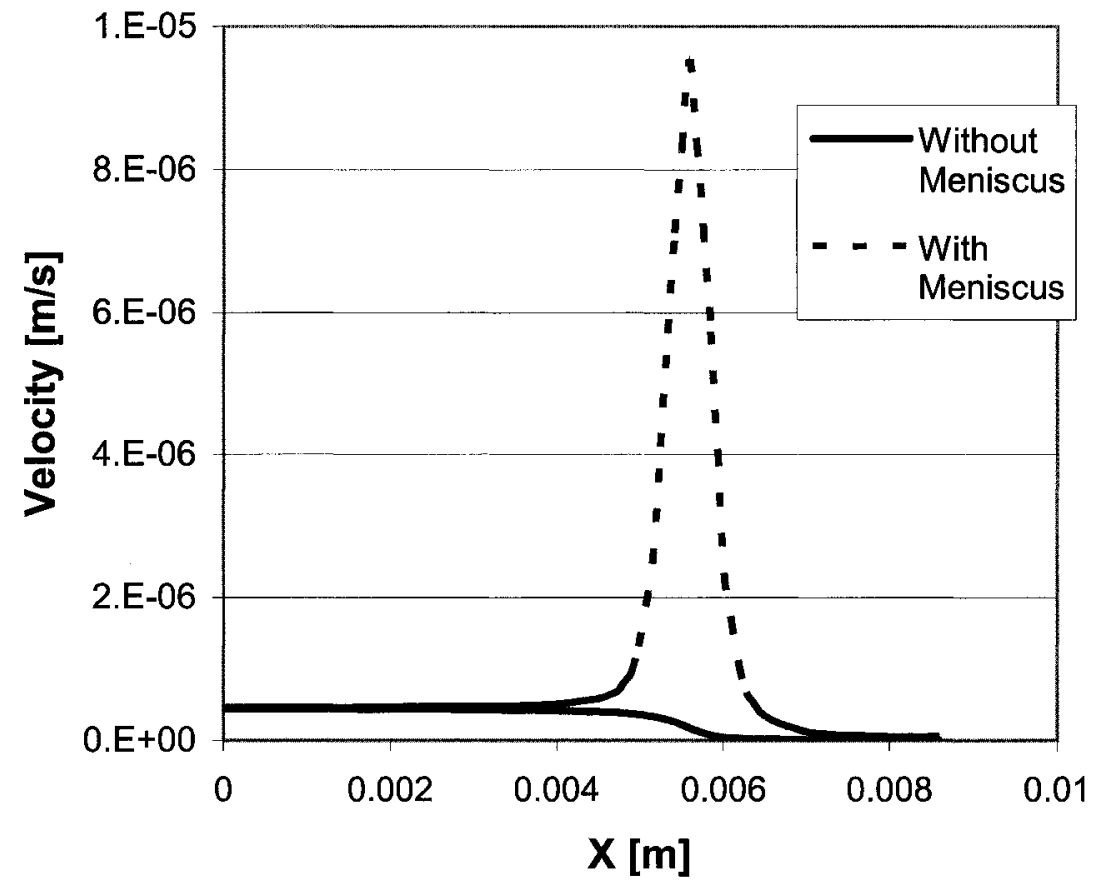

Figure 5.13 Liquid velocity at evaporative surface comparison (30 $\mathrm{W}$ applied heat flux heating fin lies on $x=0.0057 \mathrm{~m}-0.0086 \mathrm{~m}$ at $\mathrm{y}=0.0072 \mathrm{~m}$ ) 


\subsubsection{Temperature Field}

The temperature profile of the evaporator when modelling with the addition of the meniscus greatly resembles that without meniscus as described in Section 5.3.2.

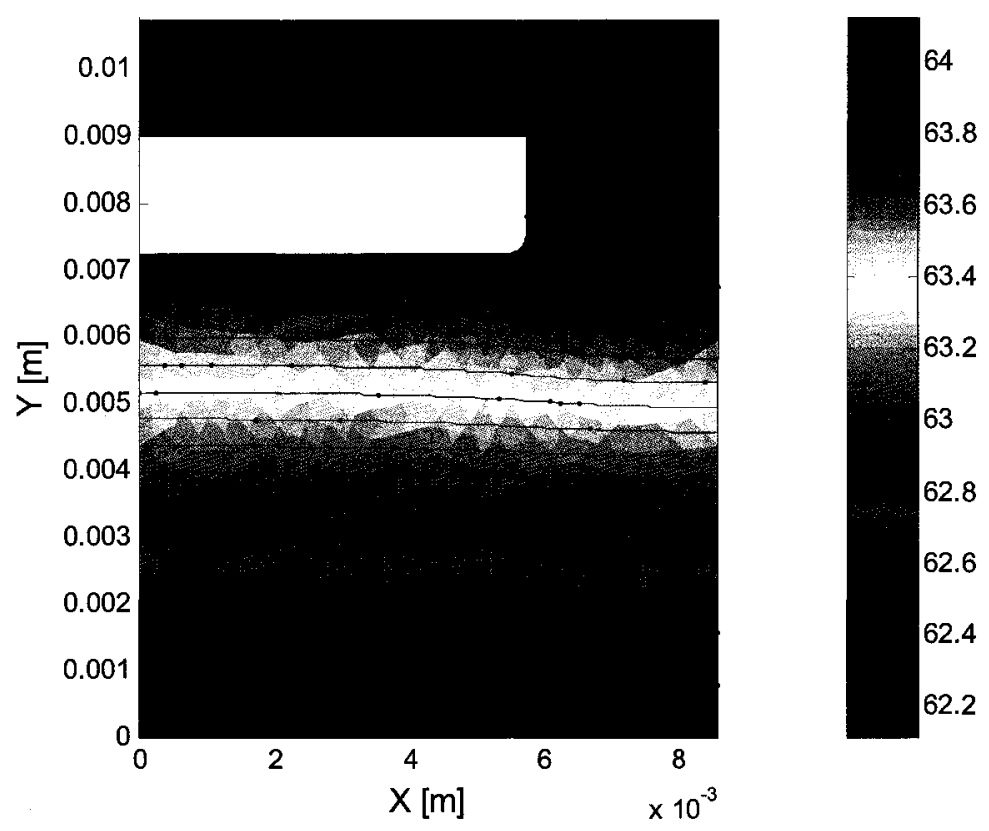

Figure 5.14 Temperature field $\left[{ }^{\circ} \mathrm{C}\right]$ with meniscus (30 W applied heat flux)

However, there are some subtle differences that affect the overall loop heat pipe operation. The vectors on Figure 5.15 demonstrate that the meniscus will not only accept heating from the saturated wick but will also be directly heated by the heating fin. This effect has two results. Firstly, it increases the incoming thermal flux to the already larger surface area of the evaporative surface therefore inducing greater evaporation rates as also suggested by [11]. The increase in evaporation has tendencies to reduce the casing temperature through the increased amount of energy release, though this effect is minimal. Secondly, the presence of the meniscus increases the thermal resistance at the surface of the wick at its basal location because of its lower thermal conductivity (pure 
fluid) and its higher temperature from fin heating. This effect forces the horizontal extension of the thermal path for the flux entering at the fin-wick interface to reach the wick's unblocked surface. This latter effect can be seen through the isotherms of Figure 5.15 and has little effect to the operation of the loop heat pipe.

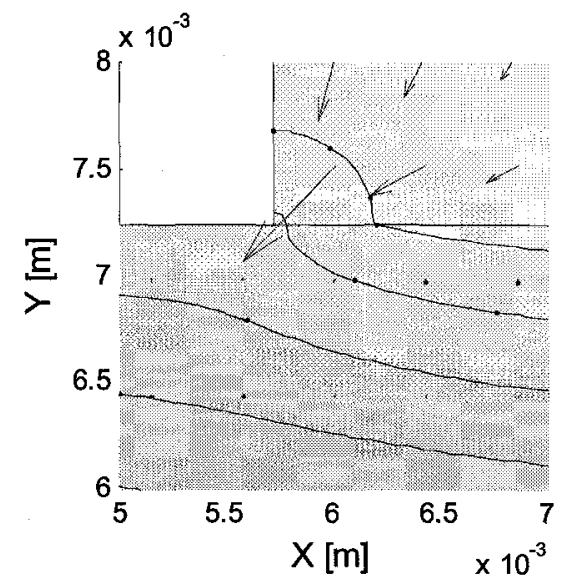

a)

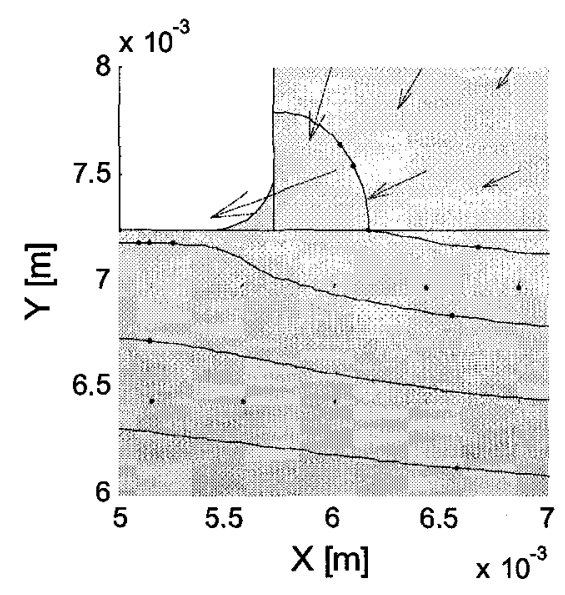

b)

Figure 5.15 Investigation of thermal flux in region meniscus: a) Meniscus absent, b) Meniscus present

\subsection{Choice of Vapour Superheat}

It was observed that the prescribed superheat trend effectively adjusts the evaporator's overall temperature to the same trend as shown in Figure 5.16 for both scenarios examined. Furthermore, when the vapour temperature is prescribed as the evaporative boundary condition, as mentioned in Section 5.3.1, the amount of superheat dictates the surface evaporation intensity trend. A decrease in superheat will increase the evaporation intensity bias towards the region nearest the fin. These effects emphasizes that special attention should be taken to ensure that the prescribed superheat trend reasonably predicts that of a localized evaporator cross section. 


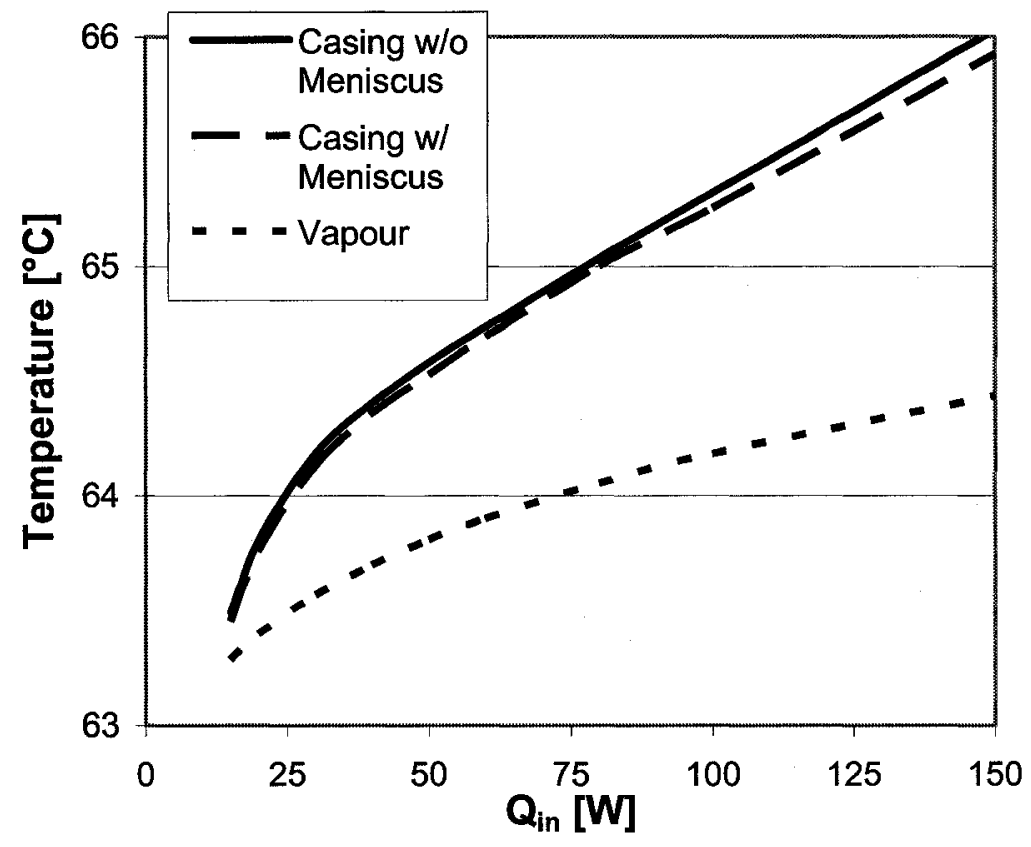

Figure 5.16 Effect of superheat trend on casing temperature 


\section{Chapter 6: Conclusions}

The work presented in this thesis achieves the objective of developing a two-dimensional steady state evaporator model. This evaporator model is intended to investigate flow properties of two speculated internal scenarios for operation at low heat fluxes: 1) a fully saturated wick and casing and 2) a fully saturated wick and casing with the addition of excess fluid accumulated in the vapour groove causing the formation of a meniscus where the heating fin makes contact with the wick. The geometry being evaluated is that of a flat type evaporator.

The evaporator model consisting of four sub-models was programmed to determine pressure, velocity, and temperature profiles. The four sub-models were independently designed to address specific evaporator parameters: a diffusive thermal model for the casing and meniscus, a convective-diffusive thermal model for the wick, a Laplace solver to evaluate the wick's pressure field and a Darcy flow solver to convert pressure field to velocity field in the wick. Conservation of temperature and flux at the casing-wick, casing-meniscus, and wick-meniscus interfaces was achieved through simultaneous solutions of the thermal sub-models thereby solving all domains as a continuous thermal field. The Laplace and Darcy sub-models were solved independently and related back to the convective-diffusive thermal sub-model through a developed iterative procedure. 
Within these four sub-models, governing equations with their appropriate boundary conditions were solved using a finite element solver (MATLAB - PDETool) requiring modification and workarounds which are described within the thesis. A porous media channel test validated the convection and pressure fields of the evaporator model with CFX results. Additionally, a qualitative analysis on a tank with an open face demonstrated that the model results are in agreement with the expected evaporation physical behaviour.

Results from the evaporator model for both scenarios 1 and 2 qualitatively agree to reported trends in literature. From the results the following was concluded:

\section{Scenario 1:}

1. The pressure field within the saturated wick is entirely determined by evaporation occurring at the liquid-vapour interface.

2. There are two sources of heat which induce evaporation at the liquid-vapour interface: heat transferred from the heating fin into the wick and heat transferred from the superheated vapour to the interface.

3. The convection term within the thermal equation for the wick can be neglected with little loss of accuracy because of low Peclet number $\left(P e=q_{\mathrm{in}} H_{\mathrm{w}} C_{\mathrm{p}} / h_{\mathrm{fg}} \lambda_{\mathrm{c}}\right)$.

4. The thermal flux entering the wick from the fin undergoes curvature therefore demonstrates a sign of two-dimensionality. This emphasises the need to model the casing with the wick to adequately model the temperature field. 
Scenario 2:

1. The addition of the meniscus increases the localized evaporation rate by two means: increasing the evaporative surface and directly accepting thermal flux from the heating fin.

2. The increase in evaporation caused by the meniscus will slightly reduce the casing temperature through the increased amount of energy absorbed.

\subsection{Recommendations}

The development of the evaporator model as presented in this thesis is part of an ongoing project. Eventually, this model will be amalgamated with the rest of the loop heat pipe geometry which includes the condenser, the liquid line and compensation chamber. As experimental data is made available, through the process of validation, this model will undergo some refinement and optimization to better represent physical reality. Experimental results which would benefit this model includes experimentation on vapour temperature and pressure along the vapour groove as well as experimentation on the effects of heat flux and low pressure on the meniscus' wetting angle to a vertical wall.

Meanwhile, there are some additions that can be made to this model. The first recommended addition is the capability of modelling the vapour pocket within the wick thereby enabling the prediction of flow properties with high applied fluxes. This would require the addition of interface location prediction capability within the wick using an additional iterative process. Though difficult, the second recommendation is the addition of time dependency. This would be beneficial in investigating the changes from one applied heat load to another. 


\section{References}

[1] S.W. Chi, "Heat Pipe Theory and Practice: A Sourcebook", New York: McGrawHill, (1976).

[2] Amir Faghri, "Heat Pipe Science and Technology", New York: Taylor \& Francis, (1995).

[3] T. Kaya, T.T. Hoang, "Mathematical Modeling of Loop Heat Pipes and Experimental Validation", J. Thermophys. Heat Transfer 13 (3) (1999) 314-320.

[4] Y. F. Maidanik, S. Vershinin, V. Kholodov, and J. Dolggirev, "Heat Transfer Apparatus," U.S. Patent 4515209, May 1985.

[5] M. Kaviany, "Mechanical Engineering Series: Principles of Heat Transfer in Porous Media", New York: Springer-Verlag, (1991).

[6] I.H. Tavman, "Effective Thermal Conductivity of Granular Porous Materials", Int. Comm. Heat Mass Transfer 23 (2) (1996) 169-176.

[7] J.C. Maxwell, "A Treatise on Electricity and Magnetism: $3^{\text {rd }}$ Ed.", New York: Dover, (1954).

[8] P.Zehner, E.U. Schlunder, "Waermeleitfahigkeit won Schuettungen be Massigen Temperaturea", Chemie-Ingr-Techn 42 (1970) 933-941. 
[9] P.X. Jiang, X.C. Lu, "Numerical Simulation of Fluid Flow and Convection Heat Transfer In Sintered Porous Plate Channels", Int. J. Heat Mass Transfer 49 (9-10) (2006) 1685-1695.

[10] M. Sahraoui, M. Kaviany, "Slip and No-Slip Boundary Conditions at the Interface of Porous, Plain Media: Convection", Int. J. Heat Mass Transfer 37 (6) (1994) 10291044.

[11] A.S. Demidov, E.S. Yatsenko, "Investigation of Heat and Mass Transfer in the Evaporation Zone of a Heat Pipe Operating by the 'Inverted Meniscus' Principle”, Int. J. Heat Mass Transfer 37 (14) (1994) 2155-2163.

[12] Y. Cao, A. Faghri, "Conjugate Analysis of a Flat-Plate Type Evaporator for Capillary Pumped Loops with Three-Dimensional Vapor Flow in the Groove", Int. J. Heat Mass Transfer 37 (3) (1994) 401-409.

[13] D. Khrustalev, A. Faghri, "Heat Transfer in the Inverted Meniscus Type Evaporator at High Heat Fluxes", Int. J. Heat Mass Transfer 38 (16) (1995) 3091-3101.

[14] C. Figus, Y. Le Bray, S. Bories, M. Prat, "Heat and Mass Transfer with Phase Change in a Porous Structure Partially Heated: Continuum Model and Pore Network Simulation", Int. J. Heat Mass Transfer 42 (1999) 2557-2569.

[15] T. Kaya, J. Goldak, "Numerical Analysis of Heat and Mass Transfer in the Capillary Structure of a Loop Heat Pipe", Int. J. Heat Mass Transfer 49 (2006) 32113220 . 
[16] C. Ren, Q.-S. Wu, M.-B. Hu, "Heat Transfer with Flow and Evaporation in Loop Heat Pipe's Wick at Low or Moderate Heat Fluxes", Int. J. Heat Mass Transfer 50 (2007) 2296-2308.

[17] K. Vafai, C.L. Tien, "Boundary and Inertial Effects on Flow and Heat Transfer in Porous Media", Int. J. Heat Mass Transfer 24 (1981) 195-203.

[18] The MathWorks, Inc-MatLab, "Electronic communication - Technical Solutions", Feb 2007; http://www.mathworks.com/support/solutions/data/1-15QRS.html ?product=PD\&solution=1-15QRS, \& http://www.mathworks.com/support/solutions/ data/1-15SJV.html?product=PD\&solution=1-15SJV.

[19] MatWeb: Material Property Data, Aug 2006; "Tin Bronze", http://www.matweb.com/ search/SpecificMaterial.asp?bassnum=MCUDBW; "Water", http:/www.matweb.com/search/SpecificMaterial.asp?bassnum=DWATR0; “Copper", http://www.matweb.com/search/SpecificMaterial.asp?bassnum=AMECu00.

[20] M.J. Moran, H.N. Shapiro, "Fundamentals of Engineering Thermodynamics $-4^{\text {th }}$ ed.", New York: John Wiley \& Sons, Inc., (2000). 